\title{
Three-Component Coupling of Arynes, Aminosilanes and Aldehydes
}

\author{
Hiroto Yoshida, * Takami Morishita, Hiroyuki Fukushima, Joji Ohshita, and Atsutaka Kunai
}

Department of Applied Chemistry, Graduate School of Engineering, Hiroshima University, Higashi-Hiroshima 739-8527, Japan.

\section{Supporting Information}

General Remarks. All manipulations of oxygen- and moisture-sensitive materials were conducted with a standard Schlenk technique under a purified argon atmosphere. Nuclear magnetic resonance spectra were taken on a JEOL EX-270 $\left({ }^{1} \mathrm{H}, 270 \mathrm{MHz} ;{ }^{13} \mathrm{C}, 67.8 \mathrm{MHz}\right)$ spectrometer or a JEOL Lambda-400 $\left({ }^{1} \mathrm{H}, 400 \mathrm{MHz} ;{ }^{13} \mathrm{C}, 99.5 \mathrm{MHz}\right)$ spectrometer using residual chloroform $\left({ }^{1} \mathrm{H}\right)$ or $\mathrm{CDCl}_{3}\left({ }^{13} \mathrm{C}\right)$ as an internal standard. High-resolution mass spectra were obtained with a JEOL JMS-SX102A or JEOL JMS-HX110A spectrometer. Melting points were measured with Yanaco Micro Melting Point apparatus and uncorrected. Column chromatography was carried out using Merck Kieselgel 60. Unless otherwise noted, commercially available reagents were used without purification. 18-Crown-6 was recrystallized from distilled MeCN. KF (spray-dried) was vacuum dried at $100{ }^{\circ} \mathrm{C}$ for $12 \mathrm{~h}$. THF was distilled from sodium/benzophenone ketyl. $\mathrm{MeCN}$ was distilled from phosphorus pentoxide.

Aryne Precursors. $\quad$ 2-(Trimethylsilyl)phenyl triflate $\quad(\mathbf{1 a}),{ }^{1} \quad$ 4,5-dimethyl-2(trimethylsilyl)phenyl triflate (1b), 6-(trimethylsilyl)-5-indanyl triflate (1c), ${ }^{2}$ 3-(trimethylsilyl)5,6,7,8-tetrahydro-2-naphthyl triflate (1d), 3-(trimethylsilyl)-2-naphthyl triflate (1e), 3,6dimethyl-2-(trimethylsilyl)phenyl triflate (1f), 3,6-dimethoxy-2-(trimethylsilyl)phenyl triflate (1g), ${ }^{2}$ 4-methoxy-2-(trimethylsilyl)phenyl triflate (1h) ${ }^{4}$ 4-fluoro-2-(trimethylsilyl)phenyl triflate (1i) ${ }^{3}$ 6-methyl-2-(trimethylsilyl)phenyl triflate $(\mathbf{1 j}){ }^{4}$ and 3-methoxy-2-(trimethylsilyl)phenyl triflate $(\mathbf{1 k})^{5}$ were prepared according to literature procedures.

Aminosilanes. All aminosilanes, except for $\mathbf{2 g}$ that is commercially available, were prepared from corresponding amines and chlorotrimethylsilane according to procedure A or B as follows.

Procedure $\boldsymbol{A}$ (for $\mathbf{2 a}-\mathbf{2 c}, \mathbf{2 h}$ and $\mathbf{2 i})$ : To a $\mathrm{CH}_{2} \mathrm{Cl}_{2}(15 \mathrm{~mL})$ solution of an amine $(0.014 \mathrm{~mol})$ and triethylamine $(0.024 \mathrm{~mol})$ was added chlorotrimethylsilane $(0.012 \mathrm{~mol})$ at $0{ }^{\circ} \mathrm{C}$, and the resulting mixture was stirred at room temperature overnight. After the solvent was distilled away $(15 \mathrm{mmHg})$, dry $\mathrm{n}$-hexane $(20 \mathrm{~mL})$ was added to the residue, and the insoluble materials were filtered through a Celite plug. Concentration of the solution gave an aminosilane, which can be used for the aminosilylation without further purification. 
Procedure B (for $2 \mathbf{d}-\mathbf{2 f})$ : To a THF solution $(6 \mathrm{~mL})$ of an amine $(6.0 \mathrm{mmol})$ was added $n$-BuLi $(1.54 \mathrm{M}$ solution in $n$-hexane, $3.9 \mathrm{~mL}, 6.0 \mathrm{mmol})$ at $0{ }^{\circ} \mathrm{C}$, and the mixture was stirred at $0{ }^{\circ} \mathrm{C}$ for $0.5 \mathrm{~h}$. To this mixture was added chlorotrimethylsilane $(6.0 \mathrm{mmol})$, and the resulting mixture was stirred at room temperature overnight before the removal of the solvent in vacuo. The residue was treated with dry $n$-hexane $(20 \mathrm{~mL})$, and the insoluble materials were filtered through a Celite pad. Evaporation of the solvent gave an aminosilane of sufficient purity.

\section{Three-Component Coupling of Arynes, Aminosilanes and Aldehydes. A General} Procedure. To a THF solution $(1 \mathrm{~mL})$ of an aryne precursor $(0.18 \mathrm{mmol})$, an aminosilane $(0.15 \mathrm{mmol})$, an aldehyde $(0.225 \mathrm{mmol})$, benzoic acid $(1.8 \mathrm{mg}, 0.015 \mathrm{mmol})$ and 18 -crown-6 $(0.095 \mathrm{~g}, 0.36 \mathrm{mmol}), \mathrm{KF}(0.030 \mathrm{~g}, 0.51 \mathrm{mmol})$ was added, and the resulting mixture was stirred at $0{ }^{\circ} \mathrm{C}$ for the period as specified in Table 1 , Table 2 or eq. 2 . The mixture was diluted with ethyl acetate, filtered through a Celite plug, and concentrated. After a hexane (or toluene) solution $(5 \mathrm{~mL})$ of the residue was treated with aluminum oxide $(2.5 \mathrm{~g})$ containing a $75 \mu \mathrm{L}$ of water at room temperature overnight, ${ }^{6}$ the resulting mixture was filtered through a Celite plug. Evaporation of the solvent followed by silica-gel column chromatography (dichloromethane-ethyl acetate as an eluent) gave the corresponding product.

\section{2-(Diethylamino) benzhydrol (4a).}

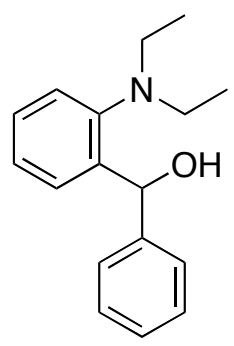

$4 a$

Isolated in 76\% yield as a pale yellow oil: ${ }^{1} \mathrm{H} \mathrm{NMR}\left(\mathrm{CDCl}_{3}\right) \delta 1.03(\mathrm{t}, J=7.2 \mathrm{~Hz}, 6 \mathrm{H}), 2.86$ 2.99 (m, 4 H), 6.02 (s, $1 \mathrm{H}), 6.97$ (d, J = 7.3 Hz, $1 \mathrm{H}), 7.11-7.14(\mathrm{~m}, 1 \mathrm{H}), 7.22-7.42(\mathrm{~m}, 7 \mathrm{H})$, 8.27 (brs, $1 \mathrm{H}) ; \quad{ }^{13} \mathrm{C} \mathrm{NMR}\left(\mathrm{CDCl}_{3}\right) \delta 12.4,49.1$, 76.1, 123.7, 125.3, 126.9, 127.8, 128.1, 129.3, 140.0, 144.5, 148.4; Anal. Calcd for $\mathrm{C}_{17} \mathrm{H}_{21} \mathrm{NO}$ : C, 79.96; H, 8.29; N, 5.49. Found: C, $80.00 ; \mathrm{H}, 8.01 ; \mathrm{N}, 5.64$.

\section{2-(Dibuthylamino)benzhydrol (4b).}




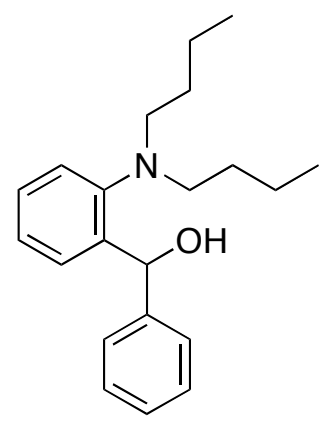

4b

Isolated in 77\% yield as a pale yellow oil: ${ }^{1} \mathrm{H} \mathrm{NMR}\left(\mathrm{CDCl}_{3}\right) \delta 0.83(\mathrm{t}, J=7.3 \mathrm{~Hz}, 6 \mathrm{H}), 1.11$ 1.29 (m, $4 \mathrm{H}), 1.37-1.48(\mathrm{~m}, 4 \mathrm{H}), 2.80(\mathrm{t}, J=7.8 \mathrm{~Hz}, 4 \mathrm{H}), 6.00(\mathrm{~s}, 1 \mathrm{H}), 7.49$ (d, $J=7.6 \mathrm{~Hz}, 1$ $\mathrm{H}), 7.08-7.14(\mathrm{~m}, 1 \mathrm{H}), 7.22-7.41(\mathrm{~m}, 7 \mathrm{H}), 8.10$ (brs, $1 \mathrm{H}) ; \quad{ }^{13} \mathrm{C} \mathrm{NMR}\left(\mathrm{CDCl}_{3}\right) \delta 13.9,20.5$, 29.4, 55.5, 76.0, 123.6, 125.0, 126.86, 126.93, 127.8, 128.1, 129.4, 139.0, 144.5, 149.5; Anal. Calcd for $\mathrm{C}_{21} \mathrm{H}_{29} \mathrm{NO}: \quad$ C, 80.98; H, 9.38; N, 4.50. Found: $\quad$ C, 80.96; H, 9.31; N, 4.39.

\section{2-[(Cyclohexyl)(methyl)amino]benzhydrol (4c).}<smiles>[C]C(C)(C)c1cccc(C(O)c2ccccc2N(C2CCCCC2)C2CCCCC2)c1</smiles>

Isolated in $68 \%$ yield as a pale yellow oil: ${ }^{1} \mathrm{H}$ NMR $\left(\mathrm{CDCl}_{3}\right) \delta 0.98-1.11(\mathrm{~m}, 3 \mathrm{H}), 1.19-1.32$ (m, $3 \mathrm{H}), 1.68-1.79$ (m, $3 \mathrm{H}), 1.89(\mathrm{~d}, J=12.1 \mathrm{~Hz}, 1 \mathrm{H}), 2.52-2.63(\mathrm{~m}, 4 \mathrm{H}), 5.98(\mathrm{~s}, 1 \mathrm{H}), 7.03$ $(\mathrm{d}, J=7.2 \mathrm{~Hz}, 1 \mathrm{H}), 7.08-7.14(\mathrm{~m}, 1 \mathrm{H}), 7.23-7.26(\mathrm{~m}, 3 \mathrm{H}), 7.33(\mathrm{t}, J=8.0 \mathrm{~Hz}, 2 \mathrm{H}), 7.40(\mathrm{~d}, J$ $=7.7 \mathrm{~Hz}, 2 \mathrm{H}), 8.18$ (brs, $1 \mathrm{H}) ;{ }^{13} \mathrm{C} \mathrm{NMR}\left(\mathrm{CDCl}_{3}\right) \delta$ 25.4, 25.7, 30.3, 38.5, 62.5, 76.2, 124.5, 125.0, 126.7, 126.9, 127.6, 128.1, 129.4, 139.0, 144.6, 150.2; Anal. Calcd for $\mathrm{C}_{20} \mathrm{H}_{25} \mathrm{NO}$ : C, 81.31; H, 8.53; N, 4.74. Found: C, 81.19; H, 8.49; N, 4.91.

\section{2-[Bis(2-methoxyethyl)amino]benzhydrol (4d).}




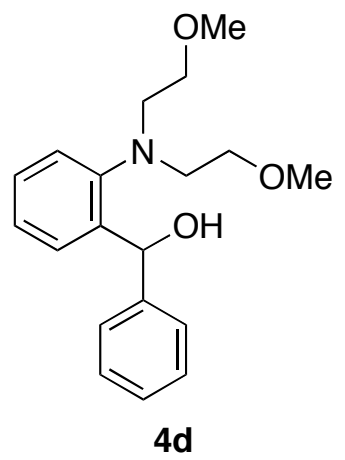

Isolated in 85\% yield as a colorless oil: ${ }^{1} \mathrm{H} \mathrm{NMR}\left(\mathrm{CDCl}_{3}\right) \delta 3.15(\mathrm{t}, J=5.6 \mathrm{~Hz}, 4 \mathrm{H}), 3.30$ (s, $6 \mathrm{H}), 3.36$ (t, $J=5.6 \mathrm{~Hz}, 4 \mathrm{H}), 6.02$ (brs, $1 \mathrm{H}), 6.19$ (s, $1 \mathrm{H}), 6.89-6.93(\mathrm{~m}, 1 \mathrm{H}), 7.08$ (td, $J=$ 7.7, $2.4 \mathrm{~Hz}, 1 \mathrm{H}), 7.22-7.43(\mathrm{~m}, 7 \mathrm{H}) ;{ }^{13} \mathrm{C} \mathrm{NMR}\left(\mathrm{CDCl}_{3}\right) \delta 55.4,58.5,70.3,72.7,124.6$, 125.6, 126.6, 126.7, 128.0, 128.2, 129.4, 142.4, 143.6, 149.3; Anal. Calcd for $\mathrm{C}_{19} \mathrm{H}_{25} \mathrm{NO}_{3}$ : C, 72.35; H, 7.99; N, 4.44. Found: C, 72.23; H, 7.84; N, 4.35.

\section{2-\{[2-(3,4-Dimethoxyphenyl)ethyl](methyl)amino\}benzhydrol (4e).}

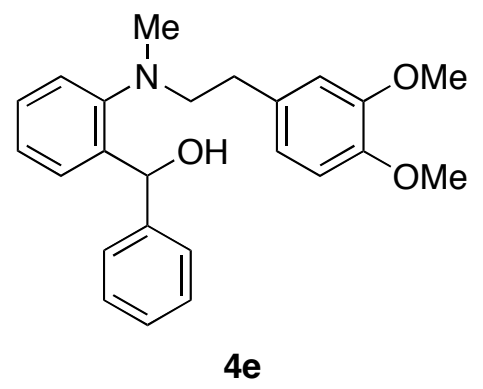

Isolated in 71\% yield as colorless oil: ${ }^{1} \mathrm{H}$ NMR $\left(\mathrm{CDCl}_{3}\right) \delta 2.63(\mathrm{~s}, 3 \mathrm{H}), 2.67-2.73(\mathrm{~m}, 2 \mathrm{H})$, 2.96-3.09 (m, 2 H), 3.84 (s, 6 H), 5.99 (s, 1 H), 6.56 (d, $J=1.9 \mathrm{~Hz}, 1 \mathrm{H}), 6.60(\mathrm{dd}, J=1.9,8.0$ $\mathrm{Hz}, 1 \mathrm{H}), 6.76(\mathrm{~d}, J=8.2 \mathrm{~Hz}, 1 \mathrm{H}), 7.10$ (d, $J=7.2 \mathrm{~Hz}, 1 \mathrm{H}), 7.14-7.19$ (m, $1 \mathrm{H}), 7.23-7.43$ (m, $7 \mathrm{H}) ;{ }^{13} \mathrm{C} \mathrm{NMR}\left(\mathrm{CDCl}_{3}\right) \delta 33.8,43.8,55.84,55.86,59.8,75.3,111.2,111.9,120.4,122.8$, 125.6, 126.6, 126.9, 128.1, 128.3, 129.3, 131.6, 138.9, 144.3, 147.5, 148.8, 150.6; Anal. Calcd for $\mathrm{C}_{24} \mathrm{H}_{27} \mathrm{NO}_{3}$ : C, 76.36; H, 7.21; N, 3.71. Found: C, 76.06; H, 7.29; N, 3.79.

\section{2-\{[(2-Tetrahydrofranyl)methyl $]$ (propyl)amino\}benzhydrol (4f).}

(A mixture of two diastereomers)<smiles>CCCN(CC1CCCO1)c1ccccc1C(O)c1ccccc1</smiles> 
Isolated in $80 \%$ yield as a pale yellow oil: ${ }^{1} \mathrm{H} \mathrm{NMR}\left(\mathrm{CDCl}_{3}\right) \delta 0.79(\mathrm{t}, J=7.3 \mathrm{~Hz}), 0.83(\mathrm{~d}, J$ = 7.4 Hz), $1.26(\mathrm{~s}), 1.34-1.65(\mathrm{~m}), 1.73-1.98(\mathrm{~m}), 2.83-3.15(\mathrm{~m}), 3.70-3.90(\mathrm{~m}), 4.03-4.13(\mathrm{~m})$, $6.29(\mathrm{~s}), 6.36(\mathrm{~s}), 6.92(\mathrm{t}, J=8.0 \mathrm{~Hz}), 7.08(\mathrm{t}, J=7.1 \mathrm{~Hz}), 7.21-7.46(\mathrm{~m}) ;{ }^{13} \mathrm{C} \mathrm{NMR}\left(\mathrm{CDCl}_{3}\right) \delta$ 11.6, 20.4, 25.4, 29.7, 58.6, 59.9, 60.1, 60.7, 67.86, 67.91, 71.7, 73.1, 76.1, 77.1, 123.8, 124.3, 125.4, 125.7, 126.57, 126.64, 126.6, 126.8, 127.92, 127.97, 128.2, 129.3, 129.4, 141.3, 143.76, 143.80; Anal. Calcd for $\mathrm{C}_{21} \mathrm{H}_{27} \mathrm{NO}_{2}$ : C, 77.50; H, 8.36; N, 4.30. Found: C, 77.24; H, $8.19 ; \mathrm{N}, 4.50$.

\section{2-(Dimethylamino) benzhydrol $(4 \mathrm{~g}) .^{7}$}<smiles>CN(C)c1ccccc1C(O)c1ccccc1</smiles>

$4 \mathrm{~g}$

Isolated in 30\% yield as a colorless oil: ${ }^{1} \mathrm{H} \mathrm{NMR}\left(\mathrm{CDCl}_{3}\right) \delta 2.60(\mathrm{~s}, 6 \mathrm{H}), 5.98(\mathrm{~s}, 1 \mathrm{H}), 7.04$ $(\mathrm{d}, J=7.7 \mathrm{~Hz}, 1 \mathrm{H}), 7.13(\mathrm{td}, J=7.3,1.7 \mathrm{~Hz}, 1 \mathrm{H}), 7.23-7.35(\mathrm{~m}, 5 \mathrm{H}), 7.40(\mathrm{~d}, J=7.7 \mathrm{~Hz}, 2 \mathrm{H})$, 7.66 (brs, $1 \mathrm{H}) ;{ }^{13} \mathrm{C} \mathrm{NMR}\left(\mathrm{CDCl}_{3}\right) \delta 45.7,75.9,122.1,125.3,126.5,126.9,128.15,128.23$, 129.2, 138.5, 144.3, 151.7; Anal. Calcd for $\mathrm{C}_{15} \mathrm{H}_{17} \mathrm{NO}$ : C, 79.26; H, 7.54; N, 6.16. Found: $\quad$ C, 78.69; H, 7.52; N, 5.94 .

2-(Azepan-1-yl)benzhydrol (4h).<smiles>OC(c1ccccc1)c1ccccc1N1CCCCCC1</smiles>

$4 \mathrm{~h}$

Isolated in 46\% yield as a yellow oil: ${ }^{1} \mathrm{H}$ NMR $\left(\mathrm{CDCl}_{3}\right) \delta 1.70$ (brs, $\left.8 \mathrm{H}\right), 2.92-3.08(\mathrm{~m}, 4 \mathrm{H})$, 5.96 (s, $1 \mathrm{H}), 6.99$ (d, J = 7.8 Hz, $1 \mathrm{H}), 7.08-7.14$ (m, $1 \mathrm{H}), 7.24-7.41$ (m, $7 \mathrm{H}), 8.21$ (d, J = 7.2 $\mathrm{Hz}, 1 \mathrm{H}) ;{ }^{13} \mathrm{C} \mathrm{NMR}\left(\mathrm{CDCl}_{3}\right) \delta 26.5,28.7,58.2,76.0,124.1,125.1,126.6,126.9,128.1,128.4$, 129.1, 137.9, 144.3, 153.9; HRNS Calcd for $\mathrm{C}_{19} \mathrm{H}_{23} \mathrm{NO}: \mathrm{M}^{+}+\mathrm{H}, 282.1860$. Found: $\mathrm{m} / \mathrm{z}$ 282.1856.

2-(1-Piperidino)benzhydrol (4i) ${ }^{8}$ 
<smiles>OC(c1ccccc1)c1ccccc1N1CCCCC1</smiles>

$4 \mathbf{i}$

Isolated in 35\% yield as a yellow oil: ${ }^{1} \mathrm{H}$ NMR $\left(\mathrm{CDCl}_{3}\right) \delta 1.55-1.66(\mathrm{~m}, 6 \mathrm{H}), 2.62-2.79(\mathrm{~m}, 4$ H), 5.93 (s, $1 \mathrm{H}), 7.06-7.16(\mathrm{~m}, 2 \mathrm{H}), 7.20-7.35$ (m, $5 \mathrm{H}), 7.41$ (d, J = 7.3 Hz, $2 \mathrm{H}), 7.89$ (brs, 1 $\mathrm{H}) ;{ }^{13} \mathrm{C} \mathrm{NMR}\left(\mathrm{CDCl}_{3}\right) \delta 23.8,26.4,54.6,76.2,122.8,125.3,126.3,126.8,128.1,129.2,138.8$, 144.6, 151.5.

2-[Bis(2-methoxyethyl)amino]-4'-nitrobenzhydrol (4j).<smiles>COCCN(CCOC)c1ccccc1C(O)c1ccc([N+](=O)[O-])cc1</smiles>

Isolated in $82 \%$ yield as a pale yellow oil: ${ }^{1} \mathrm{H} \mathrm{NMR}\left(\mathrm{CDCl}_{3}\right) \delta 3.16(\mathrm{td}, J=5.5,2.2 \mathrm{~Hz}, 4 \mathrm{H})$, 3.31 (s, $6 \mathrm{H}), 3.38$ (t, $J=4.7 \mathrm{~Hz}, 4 \mathrm{H}), 6.02$ (brs, $1 \mathrm{H}), 6.38$ (s, $1 \mathrm{H}), 6.79$ (d, $J=7.5 \mathrm{~Hz}, 1 \mathrm{H})$, 7.06-7.13 (m, $1 \mathrm{H}), 7.29-7.32(\mathrm{~m}, 2 \mathrm{H}), 7.59$ (d, $J=8.5 \mathrm{~Hz}, 2 \mathrm{H}), 8.20(\mathrm{~d}, J=8.9 \mathrm{~Hz}, 2 \mathrm{H}) ;{ }^{13} \mathrm{C}$ $\operatorname{NMR}\left(\mathrm{CDCl}_{3}\right) \delta 55.7,58.5,70.2,71.5,123.2,124.9,126.1,127.2,127.5,128.9,129.1,141.8$, 146.8, 149.4, 151.6; Anal. Calcd for $\mathrm{C}_{19} \mathrm{H}_{24} \mathrm{~N}_{2} \mathrm{O}_{5}$ : C, 63.32; H, 6.71; N, 7.77. Found: C, 63.07; H, 6.69; N, 7.55.

2-[Bis(2-methoxyethyl)amino]-4'-methoxybenzhydrol (4k). 


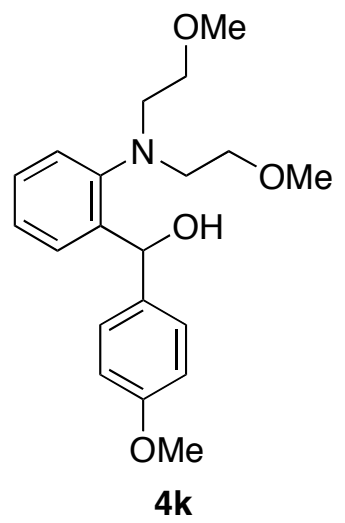

Isolated in 80\% yield as a colorless oil: ${ }^{1} \mathrm{H} \mathrm{NMR}\left(\mathrm{CDCl}_{3}\right) \delta 3.17(\mathrm{t}, J=5.4 \mathrm{~Hz}, 4 \mathrm{H}), 3.30$ (s, $6 \mathrm{H}), 3.37$ (t, $J=5.4 \mathrm{~Hz}, 4 \mathrm{H}), 3.81(\mathrm{~s}, 3 \mathrm{H}), 6.01$ (brs, $1 \mathrm{H}), 6.14(\mathrm{~s}, 1 \mathrm{H}), 6.87-6.91(\mathrm{~m}, 3 \mathrm{H})$, 7.05-7.10 (m, $1 \mathrm{H}), 7.24-7.32(\mathrm{~m}, 4 \mathrm{H}) ;{ }^{13} \mathrm{C} \mathrm{NMR}\left(\mathrm{CDCl}_{3}\right) \delta$ 55.2, 55.4, 58.5, 70.3, 72.5, 113.4, $124.5,125.6,127.9,128.2,129.3,135.8,142.5,149.2,158.5$; Anal. Calcd for $\mathrm{C}_{20} \mathrm{H}_{27} \mathrm{NO}_{4}$ : C, 69.54; H, 7.88; N, 4.05. Found: C, 69.15; H, 7.63; N, 4.45.

\section{2-[Bis(2-methoxyethyl)amino]-2',4',6'-trimethylbenzhydrol (4l).}

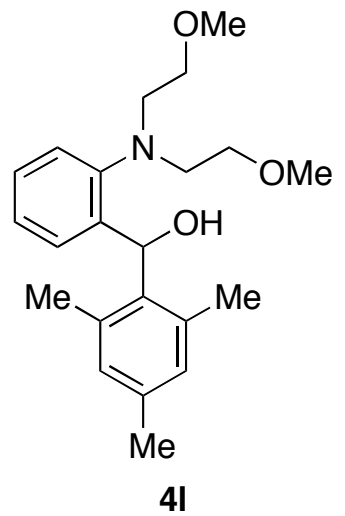

Isolated in 77\% yield as a pale yellow oil: ${ }^{1} \mathrm{H} \mathrm{NMR}\left(\mathrm{CDCl}_{3}\right) \delta 2.25(\mathrm{~s}, 6 \mathrm{H}), 2.29(\mathrm{~s}, 3 \mathrm{H})$, 3.24-3.40 (m, 4 H), 3.34 (s, 6 H), 3.45-3.56 (m, 4 H), 6.51 (s, 1 H), 6.64 (d, J = 7.7 Hz, 1 H), $6.86(\mathrm{~s}, 3 \mathrm{H}), 6.97(\mathrm{td}, J=7.5,1.2 \mathrm{~Hz}, 1 \mathrm{H}), 7.25(\mathrm{td}, J=7.5,1.1 \mathrm{~Hz}, 1 \mathrm{H}), 7.34(\mathrm{dd}, J=8.0,1.2$ $\mathrm{Hz}, 1 \mathrm{H}) ;{ }^{13} \mathrm{C} \mathrm{NMR}\left(\mathrm{CDCl}_{3}\right) \delta 20.8,21.0,55.2,58.7,70.2,70.3,124.1,125.5,127.8,127.9$, 129.7, 134.9, 136.4, 137.3, 140.2, 149.5; Anal. Calcd for $\mathrm{C}_{22} \mathrm{H}_{31} \mathrm{NO}_{3}$ : C, 73.91; $\mathrm{H}, 8.74 ; \mathrm{N}$, 3.92. Found: C, 73.95; H, 8.96; N, 4.09.

\{2-[Bis(2-methoxyethyl)amino]phenyl\}(phenanthren-9-yl)methanol (4m). 


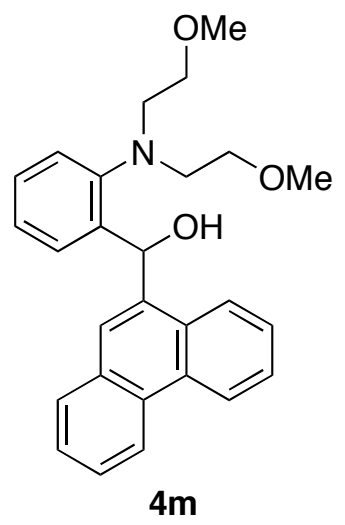

Isolated in $76 \%$ yield as a pale yellow solid: ${ }^{1} \mathrm{H}$ NMR $\left(\mathrm{CDCl}_{3}\right) \delta 3.39-3.47(\mathrm{~m}, 10 \mathrm{H}), 3.55$ 3.67 (m, $4 \mathrm{H}), 6.10$ (brs, $1 \mathrm{H}), 6.71$ (d, $J=7.7 \mathrm{~Hz}, 1 \mathrm{H}), 6.87$ (t, $J=7.4 \mathrm{~Hz}, 1 \mathrm{H}), 7.00$ (s, $1 \mathrm{H})$, $7.23(\mathrm{~d}, J=8.0 \mathrm{~Hz}, 1 \mathrm{H}), 7.36(\mathrm{~d}, J=7.5 \mathrm{~Hz}, 1 \mathrm{H}), 7.43(\mathrm{t}, J=7.2 \mathrm{~Hz}, 1 \mathrm{H}), 7.56-7.70(\mathrm{~m}, 3 \mathrm{H})$, $7.73(\mathrm{~d}, J=8.5 \mathrm{~Hz}, 1 \mathrm{H}), 7.98(\mathrm{~d}, J=7.5 \mathrm{~Hz}, 1 \mathrm{H}), 8.25(\mathrm{~s}, 1 \mathrm{H}), 8.70(\mathrm{~d}, J=8.0 \mathrm{~Hz}, 1 \mathrm{H}), 8.75$ $(\mathrm{d}, J=8.2 \mathrm{~Hz}, 1 \mathrm{H}) ;{ }^{13} \mathrm{C} \mathrm{NMR}\left(\mathrm{CDCl}_{3}\right) \delta 29.7,55.6,58.7,58.9,68.9,70.6,122.3,123.0,124.0$, 125.2 , 125.6, 125.7, 125.8, 126.1, 126.3, 126.6, 128.4, 128.93, 128.97, 129.3, 130.0, 130.2, 130.5, 131.8, 136.5, 141.8, 149.7; Anal. Calcd for $\mathrm{C}_{27} \mathrm{H}_{29} \mathrm{NO}_{3}$ : C, 78.04; H, 7.03; N, 3.37. Found: $\quad$ C, 77.99; H, 7.07; N, 3.28.

\section{2-[Bis(2-methoxyethyl)amino]-4'-cyanobenzhydrol (4n).}<smiles>COCCN(CCOC)c1ccccc1C(O)c1ccc(C#N)cc1</smiles>

Isolated in $88 \%$ yield as a yellow oil: ${ }^{1} \mathrm{H} \mathrm{NMR}\left(\mathrm{CDCl}_{3}\right) \delta 3.14(\mathrm{td}, J=5.5,1.9 \mathrm{~Hz}, 4 \mathrm{H}), 3.30$ $(\mathrm{s}, 6 \mathrm{H}), 3.35$ (t, $J=5.3 \mathrm{~Hz}, 4 \mathrm{H}), 6.00$ (brs, $1 \mathrm{H}), 6.30$ (s, $1 \mathrm{H}), 6.79$ (d, J = $7.5 \mathrm{~Hz}, 1 \mathrm{H}), 7.06-$ $7.13(\mathrm{~m}, 1 \mathrm{H}), 7.28-7.31(\mathrm{~m}, 2 \mathrm{H}), 7.53(\mathrm{~d}, J=8.2 \mathrm{~Hz}, 2 \mathrm{H}), 7.63(\mathrm{~d}, J=8.5 \mathrm{~Hz}, 2 \mathrm{H}) ;{ }^{13} \mathrm{C}$ $\operatorname{NMR}\left(\mathrm{CDCl}_{3}\right) \delta 55.6,58.4,70.2,71.7,110.4,119.1,124.9,126.0,127.4,128.8,129.2,131.8$, 141.8, 149.43, 149.49; Anal. Calcd for $\mathrm{C}_{20} \mathrm{H}_{24} \mathrm{~N}_{2} \mathrm{O}_{3}$ : C, 70.56; H, 7.11; N, 8.23. Found: C, 70.26; H, 7.07; N, 8.40.

\section{2-[Bis(2-methoxyethyl)amino]-4'-(methoxycarbonyl)benzhydrol (40).}




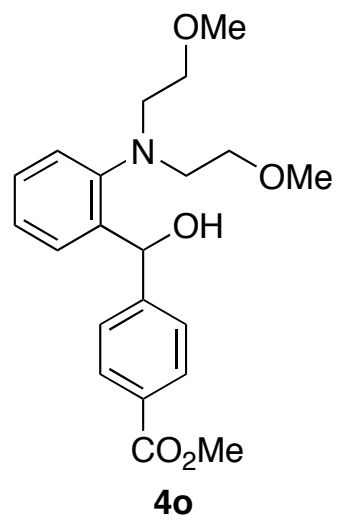

Isolated in $87 \%$ yield as a pale yellow oil: ${ }^{1} \mathrm{H} \mathrm{NMR}\left(\mathrm{CDCl}_{3}\right) \delta 3.14(\mathrm{~d}, J=5.3 \mathrm{~Hz}, 4 \mathrm{H}), 3.30$ $(\mathrm{s}, 6 \mathrm{H}), 3.35$ (t, $J=5.3 \mathrm{~Hz}, 4 \mathrm{H}), 3.90$ (s, $3 \mathrm{H}), 6.02$ (brs, $1 \mathrm{H}), 6.27(\mathrm{~s}, 1 \mathrm{H}), 6.84$ (d, J = 7.2 $\mathrm{Hz}, 1 \mathrm{H}), 7.04-7.11(\mathrm{~m}, 1 \mathrm{H}), 7.24-7.31(\mathrm{~m}, 2 \mathrm{H}), 7.48$ (d, J = 8.2 Hz, $2 \mathrm{H}), 8.01$ (d, J= 8.5 Hz, $2 \mathrm{H}) ;{ }^{13} \mathrm{C} \mathrm{NMR}\left(\mathrm{CDCl}_{3}\right) \delta 51.9,55.5,58.5,64.4,70.2,72.2,124.8,125.8,126.3,126.6,128.5$, 129.3, 129.7, 142.0, 149.1, 149.4, 167.1; Anal. Calcd for $\mathrm{C}_{21} \mathrm{H}_{27} \mathrm{NO}_{5}$ : C, 67.54; H, 7.29; N, 3.75. Found: C, 67.56; H, 7.31; N, 4.00.

2-[Bis(2-methoxyethyl)amino]-3'-bromobenzhydrol (4p).<smiles>COCCN(CCOC)c1ccccc1C(O)c1cccc(Br)c1</smiles>

Isolated in 62\% yield as a pale yellow oil: ${ }^{1} \mathrm{H} \mathrm{NMR}\left(\mathrm{CDCl}_{3}\right) \delta 3.14(\mathrm{td}, J=5.5,1.9 \mathrm{~Hz}, 4 \mathrm{H})$, 3.30 (s, $6 \mathrm{H}), 3.35$ (t, $J=5.3 \mathrm{~Hz}, 4 \mathrm{H}), 6.02$ (brs, $1 \mathrm{H}), 6.17$ (s, $1 \mathrm{H}), 6.90(\mathrm{~d}, J=7.2 \mathrm{~Hz}, 1 \mathrm{H})$, 7.08-7.13 (m, $1 \mathrm{H}), 7.19$ (t, $J=7.8 \mathrm{~Hz}, 1 \mathrm{H}), 7.27-7.31(\mathrm{~m}, 3 \mathrm{H}), 7.38(\mathrm{~d}, J=7.7 \mathrm{~Hz}, 1 \mathrm{H}), 7.62$ $(\mathrm{s}, 1 \mathrm{H}) ;{ }^{13} \mathrm{C} \mathrm{NMR}\left(\mathrm{CDCl}_{3}\right) \delta 55.5,58.5,70.3,72.1,122.4,124.7,125.3,125.8,128.5,129.3$, 129.5, 129.7, 141.9, 144.3, 149.4; Anal. Calcd for $\mathrm{C}_{19} \mathrm{H}_{24} \mathrm{BrNO}_{3}$ : C, 57.88; H, $6.14 \mathrm{~N}$, 3.55. Found: C, 57.95; H, 6.03; N, 3.54.

$\{2-[B i s(2-m e t h o x y e t h y l) a m i n o] p h e n y l\}(2-t h i e n y l) m e t h a n o l ~(4 q)$. 


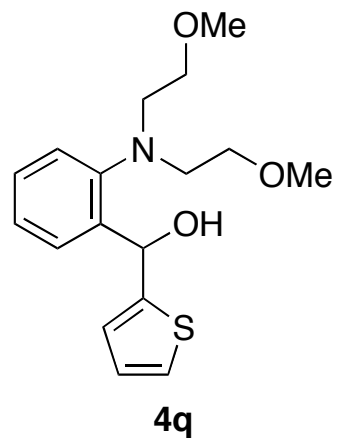

Isolated in $81 \%$ yield as a pale yellow oil: $\quad{ }^{1} \mathrm{H}$ NMR $\left(\mathrm{CDCl}_{3}\right) \delta 3.14(\mathrm{t}, J=5.7 \mathrm{~Hz}, 4 \mathrm{H}), 3.27$ $(\mathrm{s}, 6 \mathrm{H}), 3.33$ (t, $J=5.3 \mathrm{~Hz}, 4 \mathrm{H}), 6.34(\mathrm{~s}, 1 \mathrm{H}), 6.54(\mathrm{brs}, 1 \mathrm{H}), 6.79(\mathrm{dt}, J=3.4,1.2 \mathrm{~Hz}, 1 \mathrm{H})$, $6.95(\mathrm{dd}, J=5.1,3.6 \mathrm{~Hz}, 1 \mathrm{H}), 7.15-7.17(\mathrm{~m}, 2 \mathrm{H}), 7.23(\mathrm{dd}, J=5.1,1.2 \mathrm{~Hz}, 1 \mathrm{H}), 7.30-7.32(\mathrm{~m}$, $2 \mathrm{H}) ;{ }^{13} \mathrm{C} \mathrm{NMR}\left(\mathrm{CDCl}_{3}\right) \delta 55.5,58.5,70.3,70.9,77.3,123.7,124.2,124.8,125.9,126.5,128.6$, 128.9, 141.1, 148.9, 149.3; Anal. Calcd for $\mathrm{C}_{17} \mathrm{H}_{23} \mathrm{NO}_{3} \mathrm{~S}$ : C, 63.52; H, 7.21; N, 4.36. Found: $\quad$ C, 63.42; H, 7.29; N, 4.30.

1-\{2-[Bis(2-methoxyethyl)amino]phenyl\}-2,2-dimethylpropan-1-ol (4r).<smiles>COCCN(CCOC)c1ccccc1C(O)C(C)(C)Br</smiles>

Isolated in $35 \%$ yield as a pale yellow oil: ${ }^{1} \mathrm{H} \mathrm{NMR}\left(\mathrm{CDCl}_{3}\right) \delta 0.96(\mathrm{~s}, 9 \mathrm{H}), 3.17(\mathrm{t}, J=6.0$ $\mathrm{Hz}, 4 \mathrm{H}$ ), 3.29 (s, $6 \mathrm{H}$ ), 3.40 (dt, $J=9.9,6.3 \mathrm{~Hz}, 2 \mathrm{H}$ ), 3.50 (dt, $J=9.9,6.3 \mathrm{~Hz}, 2 \mathrm{H}$ ), 4.55 (brs, $1 \mathrm{H}), 4.95(\mathrm{~s}, 1 \mathrm{H}), 7.11-7.16(\mathrm{~m}, 1 \mathrm{H}), 7.23-7.28(\mathrm{~m}, 2 \mathrm{H}), 7.32(\mathrm{~d}, J=7.5 \mathrm{~Hz}, 1 \mathrm{H}) ;{ }^{13} \mathrm{C}$ NMR $\left(\mathrm{CDCl}_{3}\right) \delta$ 26.6, 36.7, 55.9, 58.5, 70.9, 77.9, 124.0, 124.7, 127.8, 129.2, 139.3, 150.7; Anal. Calcd for $\mathrm{C}_{17} \mathrm{H}_{29} \mathrm{NO}_{3}$ : C, 69.12; H, 9.89; N, 4.74. Found: C, 69.23; H, 9.91; N, 4.87 .

(E)-1-\{2-[Bis(2-methoxyethyl)amino]phenyl\}-3-phenyl-prop-2-en-1-ol (4s). 


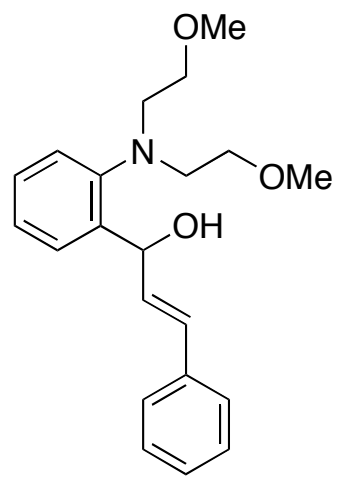

$4 \mathrm{~s}$

Isolated in $80 \%$ yield as a pale yellow oil: ${ }^{1} \mathrm{H}$ NMR $\left(\mathrm{CDCl}_{3}\right) \delta 3.24(\mathrm{t}, J=5.6 \mathrm{~Hz}, 4 \mathrm{H}), 3.31$ $(\mathrm{s}, 6 \mathrm{H}), 3.42(\mathrm{t}, J=5.6 \mathrm{~Hz}, 4 \mathrm{H}), 5.78(\mathrm{dd}, J=5.3,1.4 \mathrm{~Hz}, 1 \mathrm{H}), 6.02$ (brs, $1 \mathrm{H}), 6.50$ (dd, $J=$ 16.0, 5.3 Hz, $1 \mathrm{H}), 6.78(\mathrm{dd}, J=16.0,1.4 \mathrm{~Hz}, 1 \mathrm{H}), 7.14-7.19(\mathrm{~m}, 1 \mathrm{H}), 7.24(\mathrm{~d}, J=7.2 \mathrm{~Hz}, 1$ $\mathrm{H}), 7.28-7.34(\mathrm{~m}, 5 \mathrm{H}), 7.43(\mathrm{~d}, J=7.2 \mathrm{~Hz}, 2 \mathrm{H}) ;{ }^{13} \mathrm{C} \mathrm{NMR}\left(\mathrm{CDCl}_{3}\right) \delta 55.5,58.5,70.3,71.4$, 124.6, 125.9, 126.4, 127.2, 128.3, 128.4, 128.7, 129.7, 131.6, 137.2, 141.1, 148.9; Anal. Calcd for $\mathrm{C}_{21} \mathrm{H}_{27} \mathrm{NO}_{3}$ : C, 73.87; H, 7.97; N, 4.10. Found: C, 73.97; H, 8.01; N, 4.09.

\section{2-[Bis(2-methoxyethyl)amino]-4,5-dimethyl-4'-cyanobenzhydrol (4t).}<smiles>CCc1ccc(C(O)c2cc(C)c(C)cc2N(CCOC)CCOC)cc1</smiles>

Isolated in $85 \%$ yield as a pale yellow oil: ${ }^{1} \mathrm{H}$ NMR $\left(\mathrm{CDCl}_{3}\right) \delta 2.12(\mathrm{~s}, 3 \mathrm{H}), 2.21(\mathrm{~s}, 3 \mathrm{H})$, 3.03-3.15 (m, $4 \mathrm{H}), 3.29$ (s, $6 \mathrm{H}), 3.33$ (t, J=5.4 Hz, $4 \mathrm{H}$ ), 5.97 (brs, $1 \mathrm{H}), 6.21$ (s, $1 \mathrm{H}), 6.56$ (s, $1 \mathrm{H}), 7.04(\mathrm{~s}, 1 \mathrm{H}), 7.52(\mathrm{~d}, J=8.2 \mathrm{~Hz}, 2 \mathrm{H}), 7.65(\mathrm{~d}, J=8.2 \mathrm{~Hz}, 2 \mathrm{H}) ;{ }^{13} \mathrm{C} \mathrm{NMR}\left(\mathrm{CDCl}_{3}\right) \delta$ 19.3, 19.6, 55.8, 58.4, 70.3, 71.7, 110.2, 119.2, 125.8, 127.3, 130.2, 131.7, 134.3, 137.2, 138.9, 146.8, 150.0; Anal. Calcd for $\mathrm{C}_{22} \mathrm{H}_{28} \mathrm{~N}_{2} \mathrm{O}_{3}$ : C, 71.71; H, 7.66; N, 7.60. Found: C, $71.55 ; \mathrm{H}, 7.56 ; \mathrm{N}, 7.78$.

\section{2-[Bis(2-methoxyethyl)amino]-4,5-trimethylene-4'-cyanobenzhydrol (4u).}




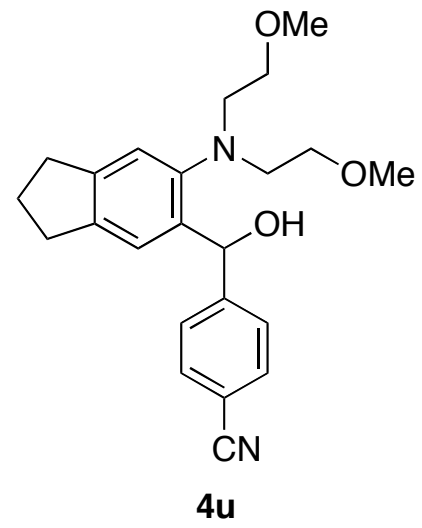

Isolated in 76\% yield as a yellow oil: ${ }^{1} \mathrm{H} \mathrm{NMR}\left(\mathrm{CDCl}_{3}\right) \delta 2.04$ (quintet, $J=7.5 \mathrm{~Hz}, 2 \mathrm{H}$ ), 2.76 $(\mathrm{t}, J=7.5 \mathrm{~Hz}, 2 \mathrm{H}), 2.86(\mathrm{t}, J=7.5 \mathrm{~Hz}, 2 \mathrm{H}), 3.06-3.19(\mathrm{~m}, 4 \mathrm{H}), 3.31(\mathrm{~s}, 6 \mathrm{H}), 3.36(\mathrm{t}, J=5.2$ Hz, 4 H), 6.13 (brs, 1 H), 6.28 (s, 1 H), 6.62 (s, 1 H), 7.15 (s, 1 H), 7.54 (d, J = 8.2 Hz, 2 H), $7.63(\mathrm{~d}, J=8.5 \mathrm{~Hz}, 2 \mathrm{H}) ;{ }^{13} \mathrm{C} \mathrm{NMR}\left(\mathrm{CDCl}_{3}\right) \delta 25.5,32.5,32.7,55.9,58.4,70.3,71.7,110.2$, 119.2, 120.4, 124.6, 127.4, 131.7, 139.6, 142.0, 145.0, 150.0; Anal. Calcd for $\mathrm{C}_{23} \mathrm{H}_{28} \mathrm{~N}_{2} \mathrm{O}_{3}$ : C, 72.60; H, 7.42; N, 7.36. Found: C, 72.64; H, 7.32; N, 7.43.

2-[Bis(2-methoxyethyl)amino]-4,5-tetramethylene-4'-cyanobenzhydrol (4v).<smiles>COCCN(CCOC)c1cc2c(cc1C(O)c1ccc(C#N)cc1)CCCC2</smiles>

Isolated in 76\% yield as a yellow solid: ${ }^{1} \mathrm{H}$ NMR $\left(\mathrm{CDCl}_{3}\right) \delta 1.69-1.80(\mathrm{~m}, 4 \mathrm{H}), 2.56-2.63(\mathrm{~m}$, $2 \mathrm{H}), 2.67-2.75$ (m, $2 \mathrm{H}), 3.07-3.14(\mathrm{~m}, 4 \mathrm{H}), 3.30$ (s, $6 \mathrm{H}), 3.34$ (t, $J=5.3 \mathrm{~Hz}, 4 \mathrm{H}), 6.00$ (brs, 1 H), $6.19(\mathrm{~s}, 1 \mathrm{H}), 6.47(\mathrm{~s}, 1 \mathrm{H}), 6.96(\mathrm{~s}, 1 \mathrm{H}), 7.53(\mathrm{~d}, J=8.2 \mathrm{~Hz}, 2 \mathrm{H}), 7.62(\mathrm{~d}, J=8.5 \mathrm{~Hz}, 2$ $\mathrm{H}) ;{ }^{13} \mathrm{C}$ NMR $\left(\mathrm{CDCl}_{3}\right) \delta 22.9,23.0,28.9,29.1,55.7,58.4,70.3,71.8,110.2,119.2,125.1$, 127.4, 129.5, 131.7, 134.8, 137.7, 138.7, 146.6, 149.9; Anal. Calcd for $\mathrm{C}_{24} \mathrm{H}_{30} \mathrm{~N}_{2} \mathrm{O}_{3}$ : C, 73.07; H, 7.66; N, 7.10. Found: C, 73.09; H, 7.84; N, 7.06.

$\{3-[\operatorname{Bis}(2-m e t h o x y e t h y l) a m i n o] n a p h t h a l e n-2-y l\}(4-c y a n o p h e n y l) m e t h a n o l ~(4 w)$. 


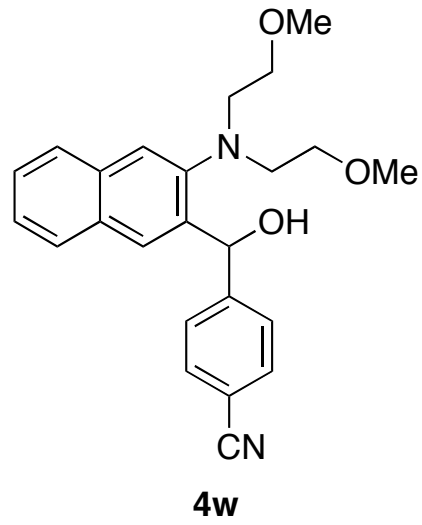

Isolated in $61 \%$ yield as a pale brown oil: ${ }^{1} \mathrm{H} \mathrm{NMR}\left(\mathrm{CDCl}_{3}\right) \delta 3.27(\mathrm{t}, J=5.3 \mathrm{~Hz}, 4 \mathrm{H}), 3.32$ $(\mathrm{s}, 6 \mathrm{H}), 3.41(\mathrm{~d}, J=5.1 \mathrm{~Hz}, 4 \mathrm{H}), 6.06$ (brs, $1 \mathrm{H}), 6.40$ (s, $1 \mathrm{H}), 7.20$ (s, $1 \mathrm{H}), 7.40$ (td, $J=6.8$, $1.2 \mathrm{~Hz}, 1 \mathrm{H}), 7.45(\mathrm{td}, J=6.8,1.2 \mathrm{~Hz}, 1 \mathrm{H}), 7.59-7.77(\mathrm{~m}, 7 \mathrm{H}) ;{ }^{13} \mathrm{C} \mathrm{NMR}\left(\mathrm{CDCl}_{3}\right) \delta 55.6$, 58.5, 58.6, 70.0, 72.06, 72.11, 110.5, 119.1, 123.2, 123.3, 125.8, 126.4, 126.7, 126.8, 127.6, 127.9, 128.5, 131.2, 131.9, 133.3, 140.6, 147.7, 149.0; HRNS Calcd for $\mathrm{C}_{24} \mathrm{H}_{26} \mathrm{~N}_{2} \mathrm{O}_{3}$ : $\mathrm{M}^{+}$, 390.1943. Found: $m / z 390.1942$.

\section{2-[Bis(2-methoxyethyl)amino]-3,6-dimethyl-4'-cyanobenzhydrol (4x).}<smiles>COCCN(CCOC)c1c(C)ccc(C)c1C(O)c1ccc(C#N)cc1</smiles>

Isolated in $60 \%$ yield as a pale yellow oil: ${ }^{1} \mathrm{H} \mathrm{NMR}\left(\mathrm{CDCl}_{3}\right) \delta 1.92(\mathrm{~s}, 3 \mathrm{H}), 2.31(\mathrm{~s}, 3 \mathrm{H})$, 3.03-3.13 (m, $1 \mathrm{H}), 3.15-3.45(\mathrm{~m}, 13 \mathrm{H}), 5.31$ (brs, $1 \mathrm{H}), 6.85(\mathrm{~d}, J=5.8 \mathrm{~Hz}, 1 \mathrm{H}), 6.91$ (d, $J=$ $7.7 \mathrm{~Hz}, 1 \mathrm{H}), 7.04(\mathrm{~d}, J=7.7 \mathrm{~Hz}, 1 \mathrm{H}), 7.39(\mathrm{~d}, J=8.2 \mathrm{~Hz}, 2 \mathrm{H}), 7.56(\mathrm{~d}, J=8.5 \mathrm{~Hz}, 2 \mathrm{H}) ;{ }^{13} \mathrm{C}$ NMR $\left(\mathrm{CDCl}_{3}\right) \delta$ 19.2, 20.2, 54.9, 56.1, 57.9, 58.6, 69.7, 72.0, 72.2, 109.4, 119.3, 126.4, 129.7, 131.5, 131.7, 134.6, 135.9, 140.9, 148.5, 151.3; Anal. Calcd for $\mathrm{C}_{22} \mathrm{H}_{28} \mathrm{~N}_{2} \mathrm{O}_{3}$ : C, 71.71; $\mathrm{H}$, 7.66; N, 7.60. Found: C, 71.58; H, 7.58; N, 7.71.

\section{2-[Bis(2-methoxyethyl)amino]-3,6-dimethoxy-4'-cyanobenzhydrol (4y).}




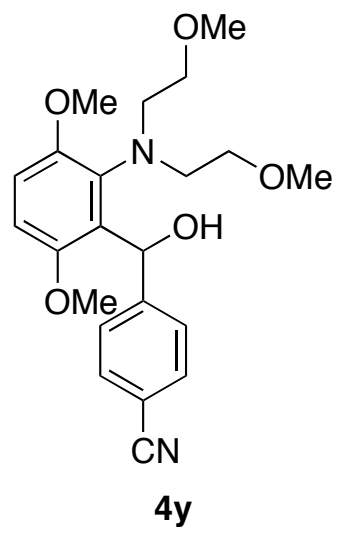

Isolated in 46\% yield as a colorless oil: ${ }^{1} \mathrm{H}$ NMR $\left(\mathrm{CDCl}_{3}\right) \delta 2.75-2.85(\mathrm{~m}, 1 \mathrm{H}), 2.91-3.07$ (m, $3 \mathrm{H}), 3.13$ (s, $3 \mathrm{H}), 3.19-3.45$ (m, $7 \mathrm{H}), 3.64$ (s, $3 \mathrm{H}), 3.78$ (s, $3 \mathrm{H}), 6.17$ (brs, $1 \mathrm{H}), 6.51$ (d, J = $9.7 \mathrm{~Hz}, 1 \mathrm{H}), 6.76(\mathrm{~d}, J=8.9 \mathrm{~Hz}, 1 \mathrm{H}), 6.81(\mathrm{~d}, J=9.2 \mathrm{~Hz}, 1 \mathrm{H}), 7.42(\mathrm{~d}, J=8.0 \mathrm{~Hz}, 2 \mathrm{H}), 7.54$ $(\mathrm{d}, J=8.2 \mathrm{~Hz}, 2 \mathrm{H}) ;{ }^{13} \mathrm{C} \mathrm{NMR}\left(\mathrm{CDCl}_{3}\right) \delta 54.2,54.7,55.3,56.0,58.6,68.5,71.0,71.1,109.5$, 109.6, 111.2, 119.3, 126.4, 131.5, 138.9, 151.1, 151.9, 153.0; Anal. Calcd for $\mathrm{C}_{22} \mathrm{H}_{28} \mathrm{~N}_{2} \mathrm{O}_{5}$ : C, 65.98; H, 7.05; N, 7.00. Found: C, 65.85; H, 7.13; N, 6.92.

A mixture of 2-[bis(2-methoxyethyl)amino]-5-methoxy-4'-cyanobenzhydrol (4z) and 2-[bis(2-methoxyethyl)amino]-4-methoxy-4'-cyanobenzhydrol (4Åfz).

(ratio: $4 \mathbf{z : 4 ' z}=$ ca. 50:50)<smiles>COCCN(CCOC)c1ccc(OC)cc1C(O)c1ccc(C#N)cc1</smiles>

$4 \mathbf{z}$<smiles>COCCN(CCOC)c1cc(OC)ccc1C(O)c1ccc(C#N)cc1</smiles>

4'z

Isolated in 67\% yield as a pale brown oil: ${ }^{1} \mathrm{H}$ NMR $\left(\mathrm{CDCl}_{3}\right) \delta$ 3.05-3.20 (m), 3.29-3.39 (m), 3.67 (s), 3.77 (s), 5.79 (brs), 6.04 (brs), 6.24-6.29 (m), 6.31 (d, $J=2.9 \mathrm{~Hz}), 6.61$ (dd, $J=2.5$, $8.7 \mathrm{~Hz}), 6.69(\mathrm{~d}, J=8.7 \mathrm{~Hz}), 6.80-6.87(\mathrm{~m}), 7.22(\mathrm{~d}, J=8.7 \mathrm{~Hz}), 7.50-7.57(\mathrm{~m}), 7.61-7.67(\mathrm{~m})$; ${ }^{13} \mathrm{C} \mathrm{NMR}\left(\mathrm{CDCl}_{3}\right) \delta 55.3,55.6,56.0,58.42,58.46,70.2,71.1,71.7,110.3,110.4,110.6,111.1$, $113.8,114.5,119.08,119.13,125.8,127.4,130.0,131.76,131.80,134.5,143.1,149.4,149.8$, 150.7, 157.1, 159.9; Anal. Calcd for $\mathrm{C}_{21} \mathrm{H}_{26} \mathrm{~N}_{2} \mathrm{O}_{4}$ : C, 68.09; H, 7.07; N, 7.56. Found: C, 67.80; H, 7.37; N, 7.65.

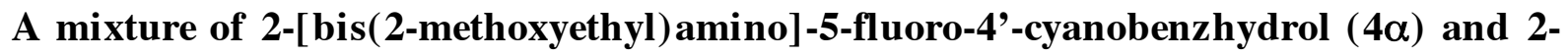
[bis(2-methoxyethyl)amino]-4-fluoro-4'-cyanobenzhydrol (4' $\alpha)$. 
(ratio: $4 \alpha: 4 ' \alpha=65: 35$ )
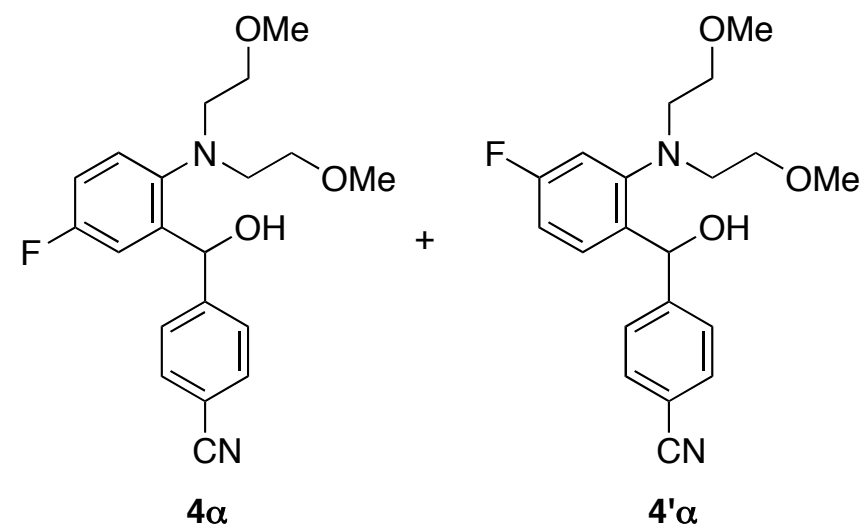

Isolated in 58\% yield as a yellow oil: ${ }^{1} \mathrm{H}$ NMR $\left(\mathrm{CDCl}_{3}\right) \delta 3.11-3.16(\mathrm{~m}), 3.31(\mathrm{~s}), 3.34-3.40$ (m), 3.77 (s), 6.32 (s), 6.34 (s), 6.44 (dd, $J=3.1,9.3 \mathrm{~Hz}), 6.71-6.81$ (m), 6.95-7.02 (m), 7.26 $(\mathrm{dd}, J=5.1,8.2 \mathrm{~Hz}), 7.50-7.55(\mathrm{~m}), 7.62-7.67(\mathrm{~m}) ;{ }^{13} \mathrm{C} \mathrm{NMR}\left(\mathrm{CDCl}_{3}\right) \delta 55.4,55.9,58.43$, 58.47, 58.49, 70.1, 70.5, 70.64, 70.67, 70.8, 110.5, 110.7, 111.9, 112.1, 112.9, 113.1, 115.44, $115.49,115.59,115.66,115.72,115.8,118.99,119.03,126.45,126.54,127.39,130.44,130.53$, 131.87, 131.94, 138.26, 138.30, 144.48, 144.54, 145.2, 148.5, 149.1, 151.2, 158.9, 161.4, 163.9; Anal. Calcd for $\mathrm{C}_{20} \mathrm{H}_{23} \mathrm{~N}_{2} \mathrm{O}_{3}$ : C, 67.01; H, 6.47; N, 7.82. Found: C, 67.09; H, 6.44; N, 7.96.

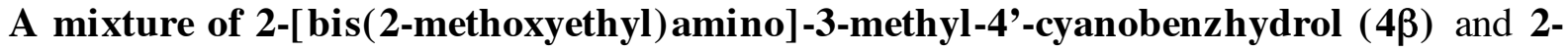
[bis(2-methoxyethyl)amino]-6-methyl-4'-cyanobenzhydrol (4' $\beta)$.

(ratio: $4 \beta: 4 ' \beta=64: 36$ )<smiles>COCCN(CCOC)c1c(C)cccc1C(O)c1ccc(C#N)cc1</smiles>

$4 \beta$<smiles>COCCN(CCOC)c1cccc(C)c1C(O)c1ccc(C#N)cc1</smiles>

$4 ' \beta$

Isolated in 64\% yield as a pale yellow oil: ${ }^{1} \mathrm{H}$ NMR $\left(\mathrm{CDCl}_{3}\right) \delta 2.26$ (s), 2.33 (s), 2.90-3.48 $(\mathrm{m}), 3.59(\mathrm{t}, J=5.6 \mathrm{~Hz}), 3.55-3.63(\mathrm{~m}), 5.60(\mathrm{~s}), 6.27(\mathrm{~s}), 6.55(\mathrm{~d}, J=7.5 \mathrm{~Hz}), 6.65(\mathrm{~s}), 6.98(\mathrm{~d}$, $J=7.6 \mathrm{~Hz}), 7.07(\mathrm{~d}, J=6.8 \mathrm{~Hz}), 7.09$ (d, $J=7.2 \mathrm{~Hz}), 7.19(\mathrm{~d}, J=7.2 \mathrm{~Hz}), 7.26(\mathrm{~d}, J=7.7 \mathrm{~Hz})$, $7.39(\mathrm{~d}, J=8.0 \mathrm{~Hz}), 7.53(\mathrm{~d}, J=8.0 \mathrm{~Hz}), 7.58(\mathrm{~d}, J=8.2 \mathrm{~Hz}), 7.63(\mathrm{~d}, J=8.2 \mathrm{~Hz}) ;{ }^{13} \mathrm{C} \mathrm{NMR}$ $\left(\mathrm{CDCl}_{3}\right) \delta 19.4,20.4,55.2,55.5,58.4,58.7,69.9,70.7,72.17,72.23,109.9,110.0,119.0,119.3$, $123.1,126.5,126.6,126.9$, 127.4, 128.5, 128.7, 131.68, 131.71, 131.9, 137.4, 137.6, 144.3, 
148.2, 150.2; Anal. Calcd for $\mathrm{C}_{21} \mathrm{H}_{26} \mathrm{~N}_{2} \mathrm{O}_{3}$ : C, 71.16; H, 7.39; N, 7.90. Found: C, $70.83 ; \mathrm{H}, 7.23 ; \mathrm{N}, 8.07$.

2-[Bis(2-methoxyethyl)amino]-6-methoxy-4'-cyanobenzhydrol $(4 \gamma)$.<smiles>COCCN(CCOC)c1cccc(OC)c1C(O)c1ccc(C#N)cc1</smiles>

Isolated in 46\% yield as a colorless oil: ${ }^{1} \mathrm{H}$ NMR $\left(\mathrm{CDCl}_{3}\right) \delta 2.80-3.05(\mathrm{~m}, 4 \mathrm{H}), 3.15-3.38$ (m, $10 \mathrm{H}), 3.80(\mathrm{~s}, 3 \mathrm{H}), 6.42(\mathrm{~d}, J=8.2 \mathrm{~Hz}, 1 \mathrm{H}), 6.72$ (brs, $1 \mathrm{H}), 6.82(\mathrm{~d}, J=8.2 \mathrm{~Hz}, 1 \mathrm{H}), 6.93$ (d, $J=8.0 \mathrm{~Hz}, 1 \mathrm{H}), 7.30(\mathrm{t}, J=8.2 \mathrm{~Hz}, 1 \mathrm{H}), 7.47(\mathrm{~d}, J=8.5 \mathrm{~Hz}, 2 \mathrm{H}), 7.55(\mathrm{~d}, J=8.5 \mathrm{~Hz}, 2 \mathrm{H})$; ${ }^{13} \mathrm{C} \mathrm{NMR}\left(\mathrm{CDCl}_{3}\right) \delta 55.0,55.7,55.8,58.5,58.6,68.3,70.0,108.3,108.5,109.7,117.1,117.2$, 119.2, 126.4, 128.4, 129.0, 131.6, 131.7, 131.8, 139.8, 150.3, 151.6, 157.5; Anal. Calcd for $\mathrm{C}_{21} \mathrm{H}_{26} \mathrm{~N}_{2} \mathrm{O}_{4}$ : C, 68.09; H, 7.07; N, 7.56. Found: C, 67.74; H, 7.16; N, 7.49.

\section{3-\{2-[Bis(2-methoxyethyl)amino]phenyl\}phtalide (9a).}

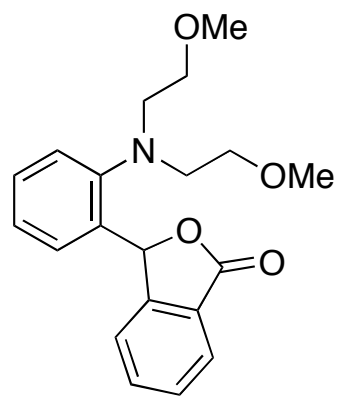

9a

Isolated in 59\% yield as a pale yellow oil: ${ }^{1} \mathrm{H} \mathrm{NMR}\left(\mathrm{CDCl}_{3}\right) \delta 3.31(\mathrm{~s}, 6 \mathrm{H}), 3.27(\mathrm{t}, J=5.3$ $\mathrm{Hz}, 4 \mathrm{H}), 3.41(\mathrm{~d}, J=5.6 \mathrm{~Hz}, 4 \mathrm{H}), 6.72(\mathrm{dd}, J=8.0,1.2 \mathrm{~Hz}, 1 \mathrm{H}), 7.05(\mathrm{td}, J=7.9,1.8 \mathrm{~Hz}, 1$ $\mathrm{H}), 7.25(\mathrm{~d}, J=7.5 \mathrm{~Hz}, 1 \mathrm{H}), 7.31-7.41(\mathrm{~m}, 3 \mathrm{H}), 7.53(\mathrm{t}, J=7.5 \mathrm{~Hz}, 1 \mathrm{H}), 7.64(\mathrm{td}, J=7.5,1.0$ $\mathrm{Hz}, 1 \mathrm{H}), 7.95(\mathrm{~d}, J=7.7 \mathrm{~Hz}, 1 \mathrm{H}) ;{ }^{13} \mathrm{C} \mathrm{NMR}\left(\mathrm{CDCl}_{3}\right) \delta 55.8,58.6,70.7,78.2,123.0,124.8$, 125.3, 125.6, 126.4 128.3, 128.8, 130.1, 134.0, 134.8, 150.8, 150.9, 171.2; Anal. Calcd for $\mathrm{C}_{20} \mathrm{H}_{23} \mathrm{NO}_{4}$ : C, 70.36; H, 6.79; N, 4.10. Found: C, 70.27; H, 6.70; N, 4.01.

3-\{2-[Bis(2-methoxyethyl)amino]phenyl\}-6,7-dimethoxyphtalide (9b). 


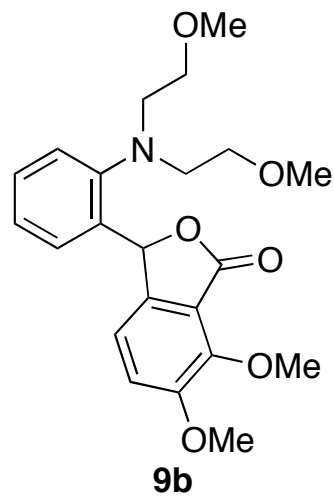

Isolated in $60 \%$ yield as a pale yellow solid: m.p. 60-63 ${ }^{\circ} \mathrm{C} ; \quad{ }^{1} \mathrm{H}$ NMR $\left(\mathrm{CDCl}_{3}\right) \delta 3.26-3.37$ $(\mathrm{m}, 10 \mathrm{H}), 3.43(\mathrm{t}, J=5.7 \mathrm{~Hz}, 4 \mathrm{H}), 3.90(\mathrm{~s}, 3 \mathrm{H}), 4.15(\mathrm{~s}, 3 \mathrm{H}), 6.79(\mathrm{~d}, J=7.7 \mathrm{~Hz}, 1 \mathrm{H}), 6.83$ $(\mathrm{d}, J=8.2 \mathrm{~Hz}, 1 \mathrm{H}), 7.02-7.10(\mathrm{~m}, 1 \mathrm{H}), 7.17(\mathrm{~d}, J=8.2 \mathrm{~Hz}, 1 \mathrm{H}), 7.22$ (s, $1 \mathrm{H}), 7.29-7.39$ (m, 2 $\mathrm{H}) ;{ }^{13} \mathrm{C} \mathrm{NMR}\left(\mathrm{CDCl}_{3}\right) \delta 55.7,56.8,58.6,62.4,70.7,117.6,118.6,119.5,124.6,125.5,128.3$, 129.9, 135.2, 144.0, 147.8, 150.7, 152.2, 168.6; Anal. Calcd for $\mathrm{C}_{22} \mathrm{H}_{27} \mathrm{NO}_{6}$ : C, 65.82; $\mathrm{H}$, 6.78; N, 3.49. Found: C, 65.78; H, 6.77; N, 3.48.

\section{3-\{2-[Bis(2-methoxyethyl)amino]phenyl $\}-6,7-($ methylenedioxy $)$ phtalide (9c).}

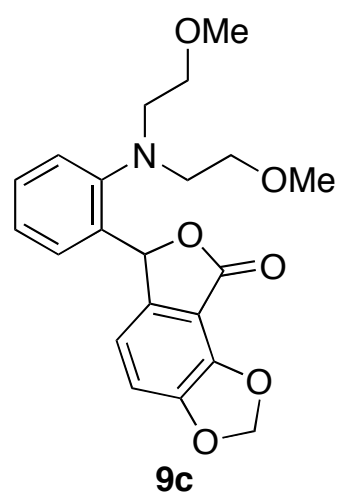

Isolated in $47 \%$ yield as a pale yellow solid: m.p. $83-85{ }^{\circ} \mathrm{C} ;{ }^{1} \mathrm{H}$ NMR $\left(\mathrm{CDCl}_{3}\right) \delta 3.26-3.36$ $(\mathrm{m}, 10 \mathrm{H}), 3.43(\mathrm{t}, J=5.3 \mathrm{~Hz}, 4 \mathrm{H}), 6.21(\mathrm{~d}, J=1.5 \mathrm{~Hz}, 2 \mathrm{H}), 6.63(\mathrm{~d}, J=8.0 \mathrm{~Hz}, 1 \mathrm{H}), 6.82(\mathrm{~d}$, $J=7.2 \mathrm{~Hz}, 1 \mathrm{H}), 7.00-7.12(\mathrm{~m}, 2 \mathrm{H}), 7.28-7.35(\mathrm{~m}, 3 \mathrm{H}) ;{ }^{13} \mathrm{C} \mathrm{NMR}\left(\mathrm{CDCl}_{3}\right) \delta$ 55.8, 58.6, 70.6, 78.7, 103.3, 109.4, 113.9, 115.5, 124.8, 125.6, 128.4, 130.1, 135.1, 143.9, 144.4, 148.8, 150.6, 168.0; Anal. Calcd for $\mathrm{C}_{21} \mathrm{H}_{23} \mathrm{NO}_{6}$ : C, 65.44; H, 6.02; N, 3.63. Found: C, 65.60; H, $6.12 ; \mathrm{N}, 3.63$.

\section{Screening of Additives for the Three-Component Coupling.}

Various additives were screened for the three-component coupling of benzyne, 2d and $\mathbf{3 1}$. Results were listed in Table A. 


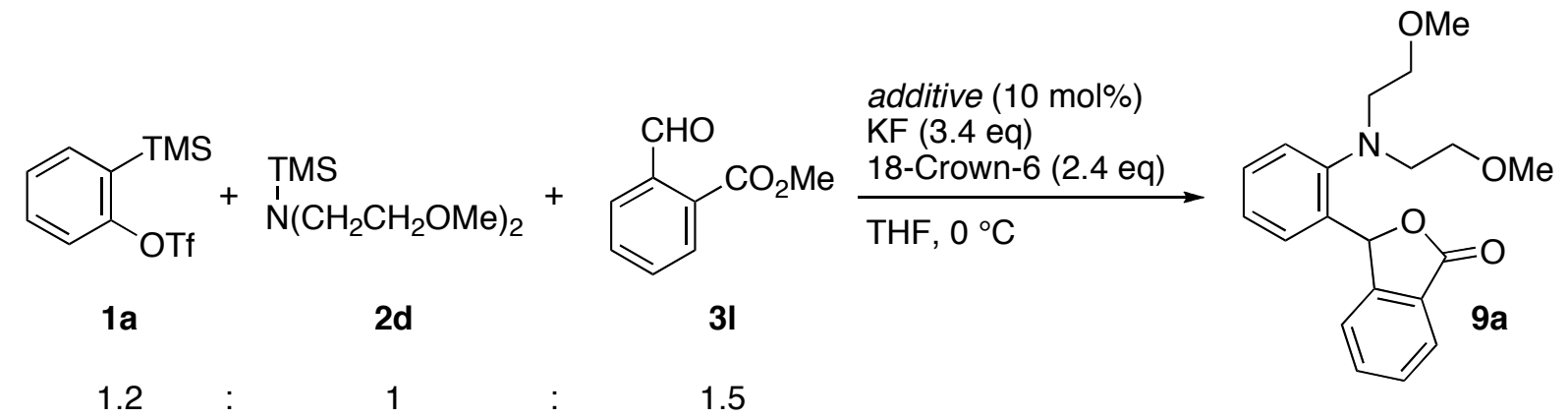

Table A

\begin{tabular}{lcc}
\hline Additive & time (h) & yield $(\%)^{a}$ \\
\hline $\mathrm{PhCO}_{2} \mathrm{H}$ & 34 & 59 \\
$\mathrm{CF}_{3} \mathrm{CO}_{2} \mathrm{H}$ & 19 & 46 \\
$\mathrm{BF}_{3} \bullet \mathrm{OEt}_{2}$ & 66 & 52 \\
$\mathrm{TMSOTf}_{\text {KOTf }}$ & 22 & 31 \\
$\mathrm{Kn}(\mathrm{OTf})_{3}$ & 24 & $16^{b}$ \\
$\mathrm{Yb}(\mathrm{OTf})_{3}$ & 12 & $27^{b}$ \\
$\mathrm{Cu}(\mathrm{OTf})_{2}$ & 6 & $18^{b}$ \\
\hline
\end{tabular}

${ }^{a}$ Yield of isolated 9a. ${ }^{b}$ NMR yield.

\section{References}

(1) Himeshima, Y.; Sonoda, T.; Kobayashi, H. Chem. Lett. 1983, 1211-1214.

(2) Yoshida, H.; Sugiura, S.; Kunai, A. Org. Lett. 2002, 4, 2767-2769.

(3) Yoshida, H.; Terayama, T.; Ohshita, J.; Kunai, A. Chem. Commun. 2004, 1980-1981.

(4) Yoshida, H.; Ikadai, J.; Shudo, M.; Ohshita, J.; Kunai, A. J. Am. Chem. Soc. 2003, 125, 6638-6639.

(5) Peña, D.; Pérez, D.; Guitián, E.; Castedo, L. J. Am. Chem. Soc. 1999, 121, 5827-5828.

(6) Feixas, J.; Capdevila, A.; Guerero, A. Tetrahedron 1994, 50, 8539-8550.

(7) Nakayama, J.; Yoshida, M.; Simamura, O. Chem. Lett. 1973, 451-454.

(8) Möhrle, H.; Gerloff, J. Arch. Pharm. (Weinheim) 1979, 312, 219-230. 
in

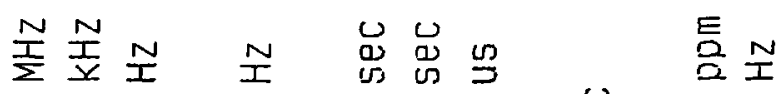

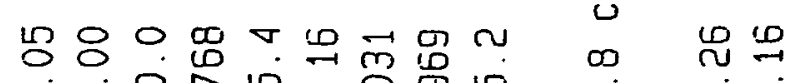

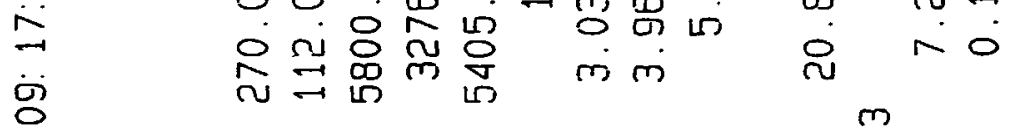

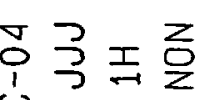

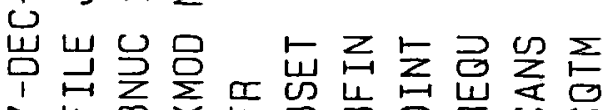

I $\stackrel{m}{\breve{0}}$

오.

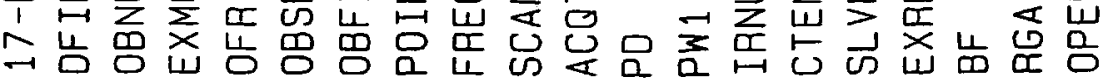

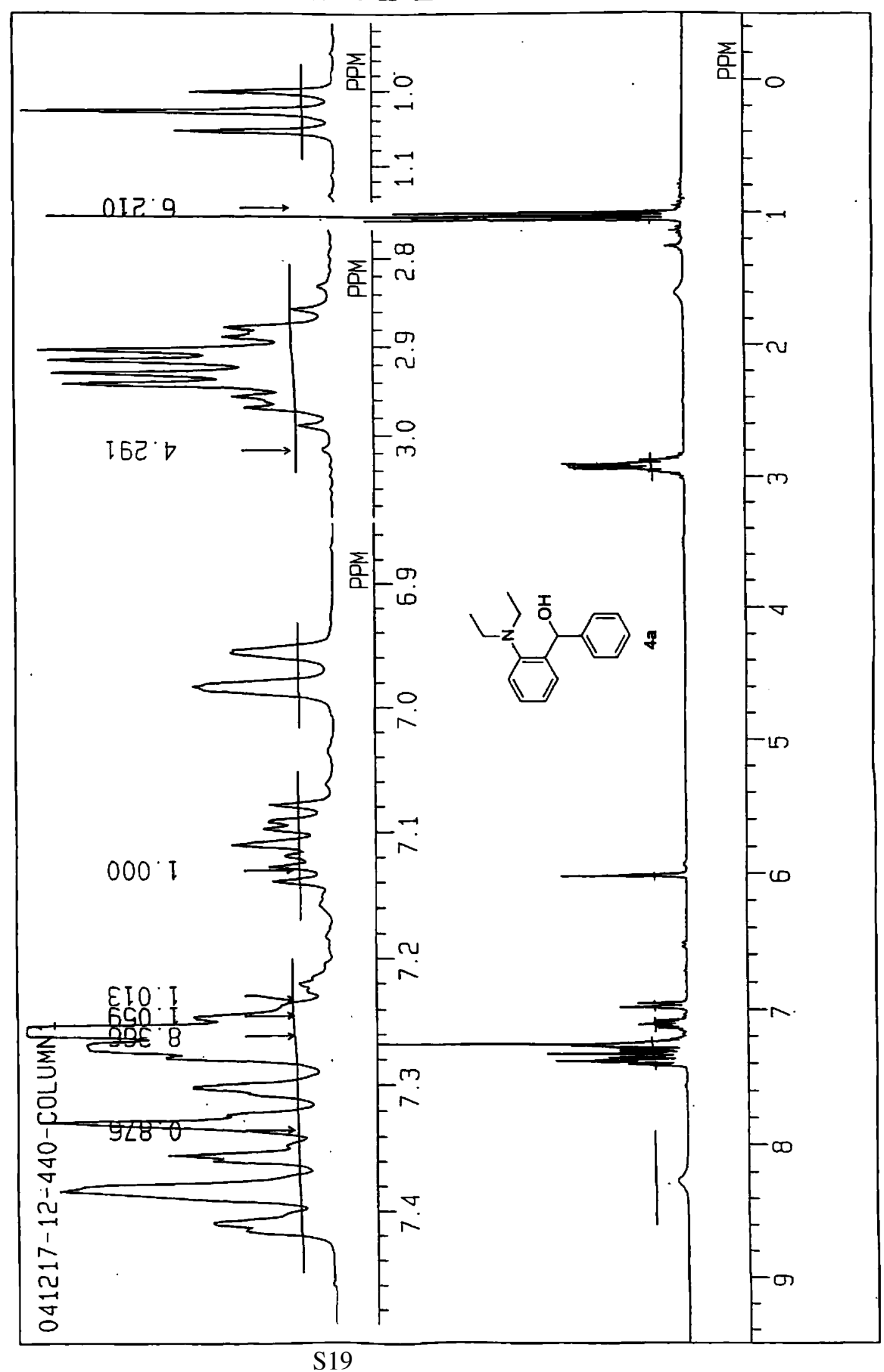




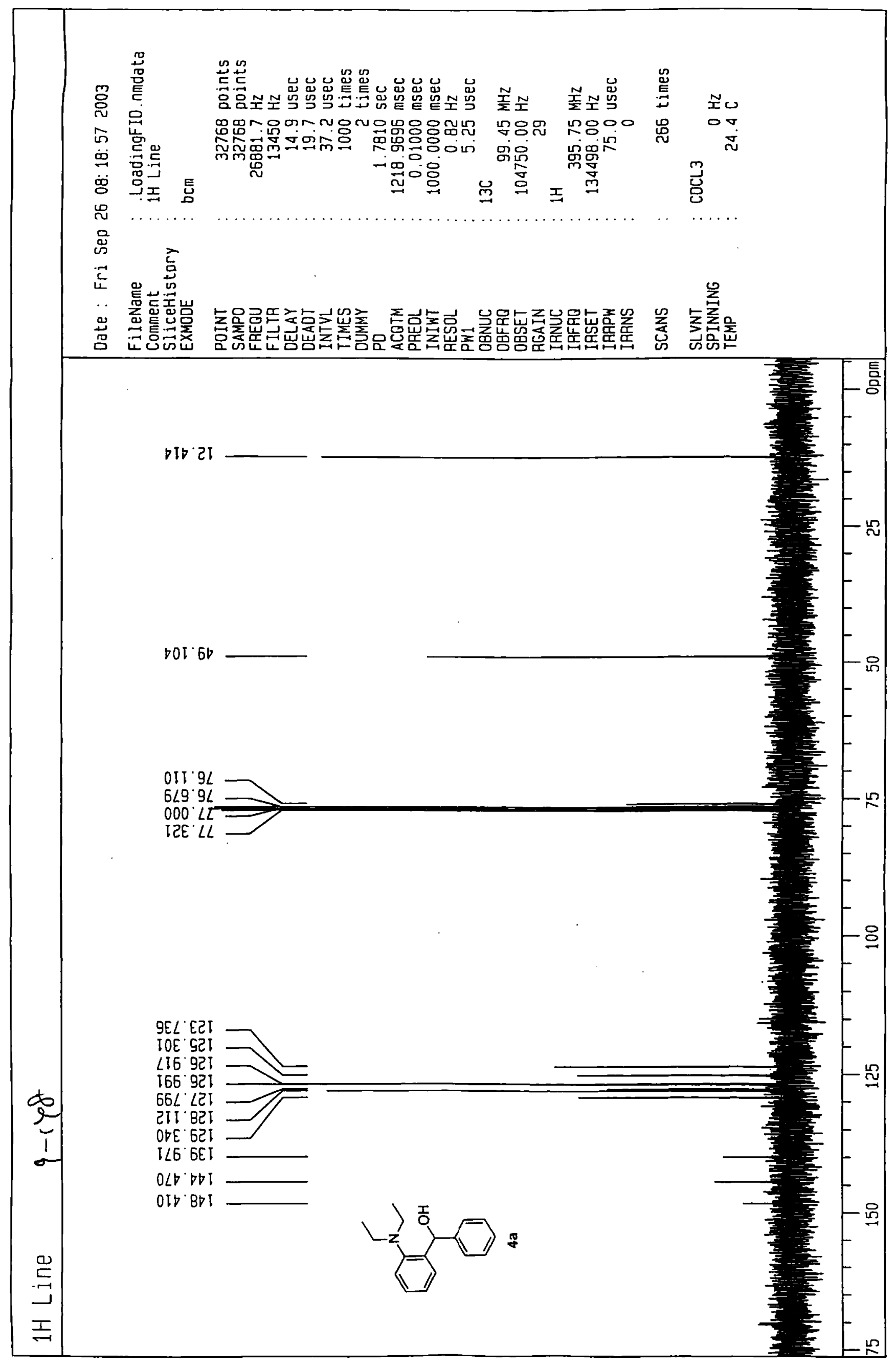




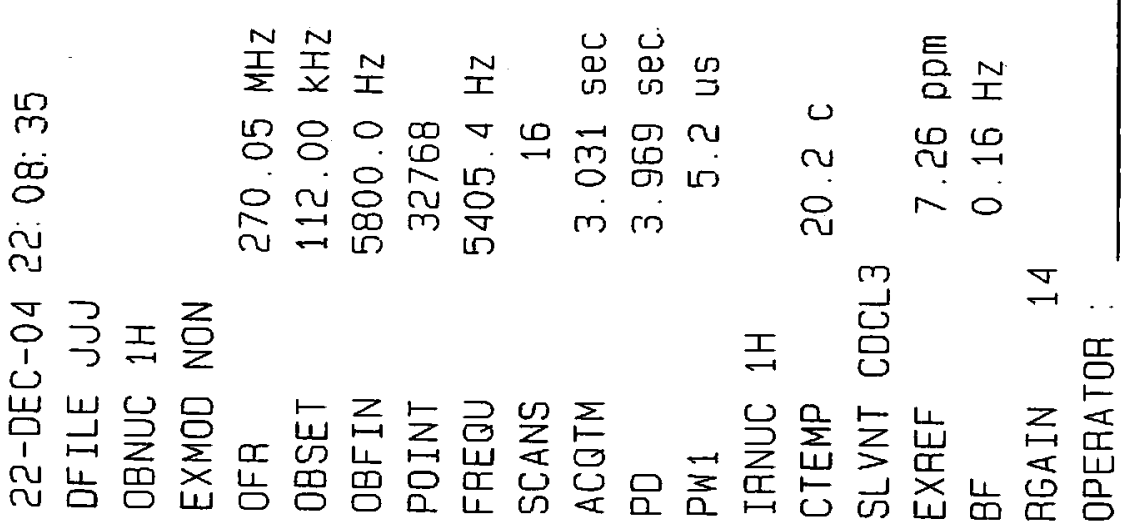

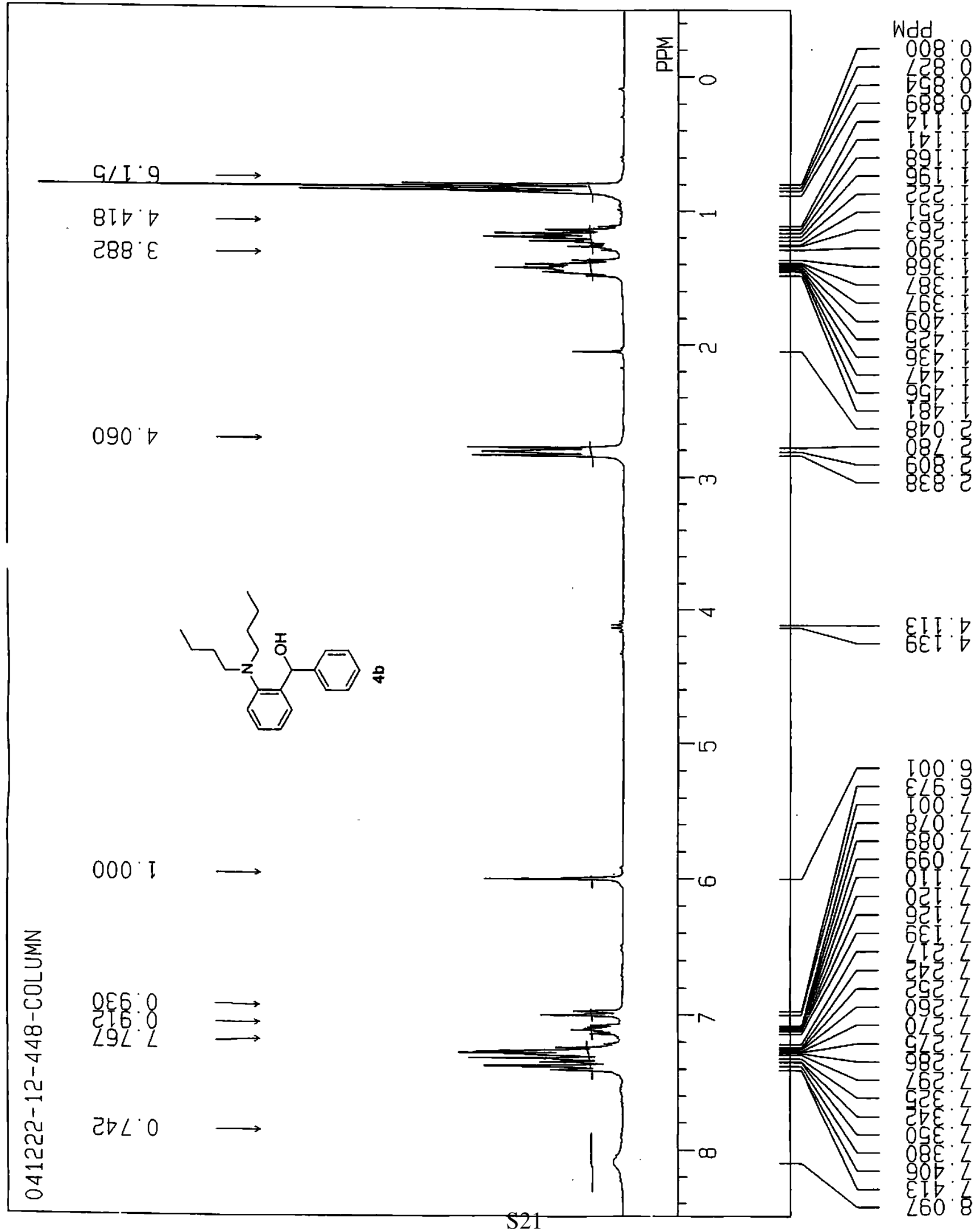


ก

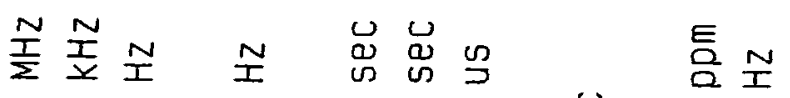

$\ddot{\dot{n}}$

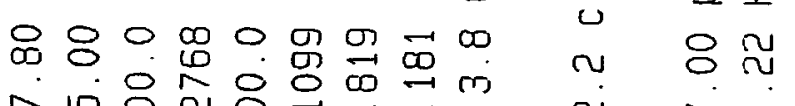

ஸे

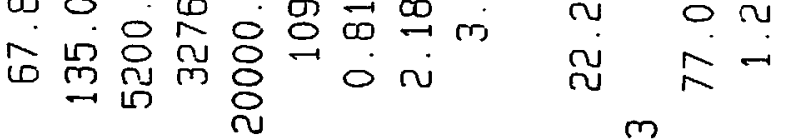

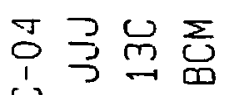

$\stackrel{m}{\mathbb{N}}$

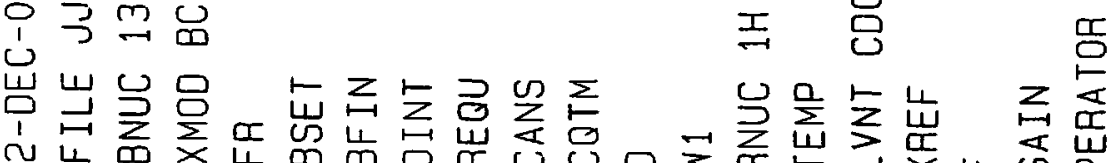

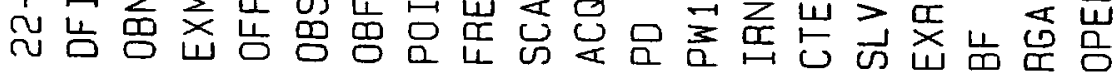

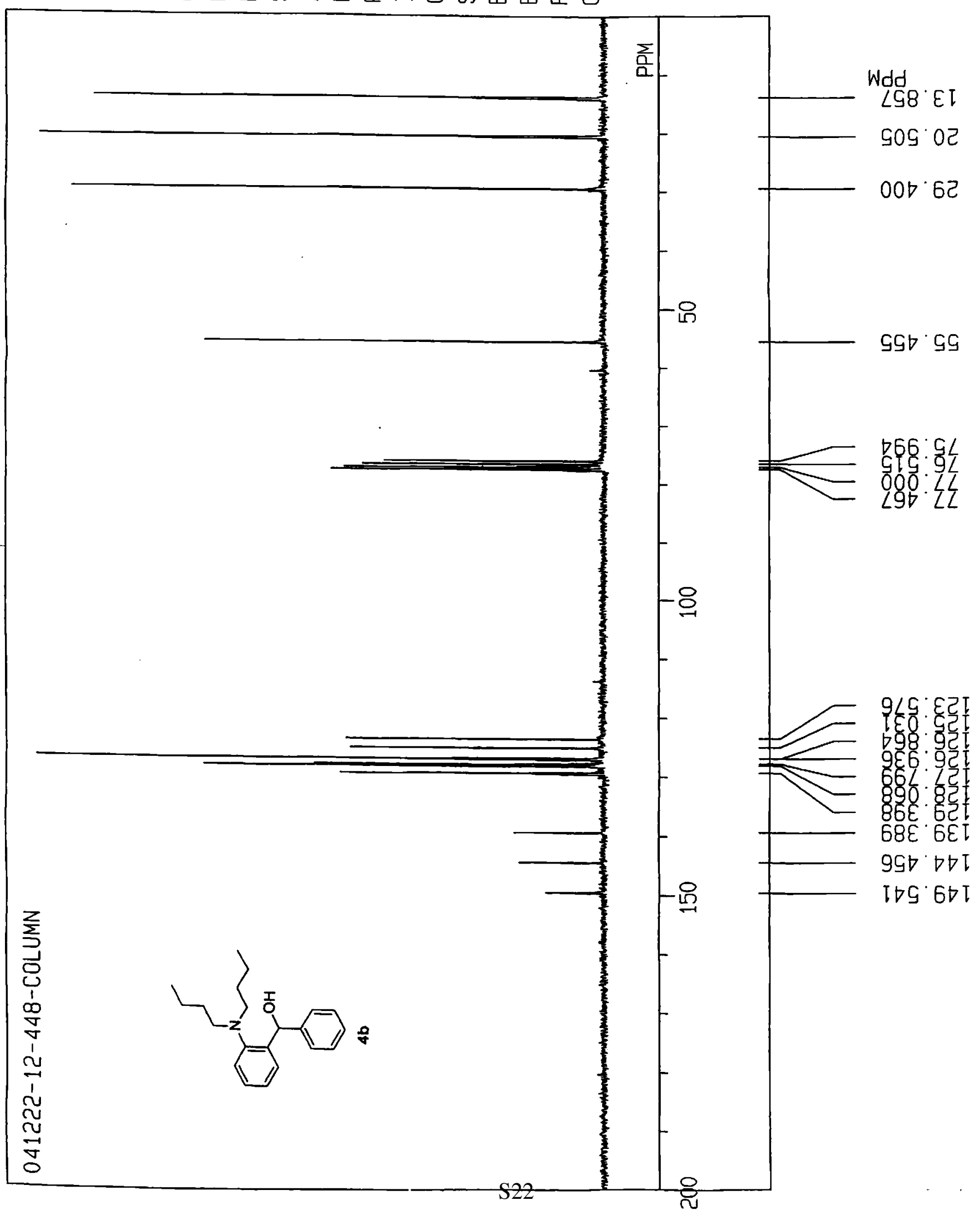




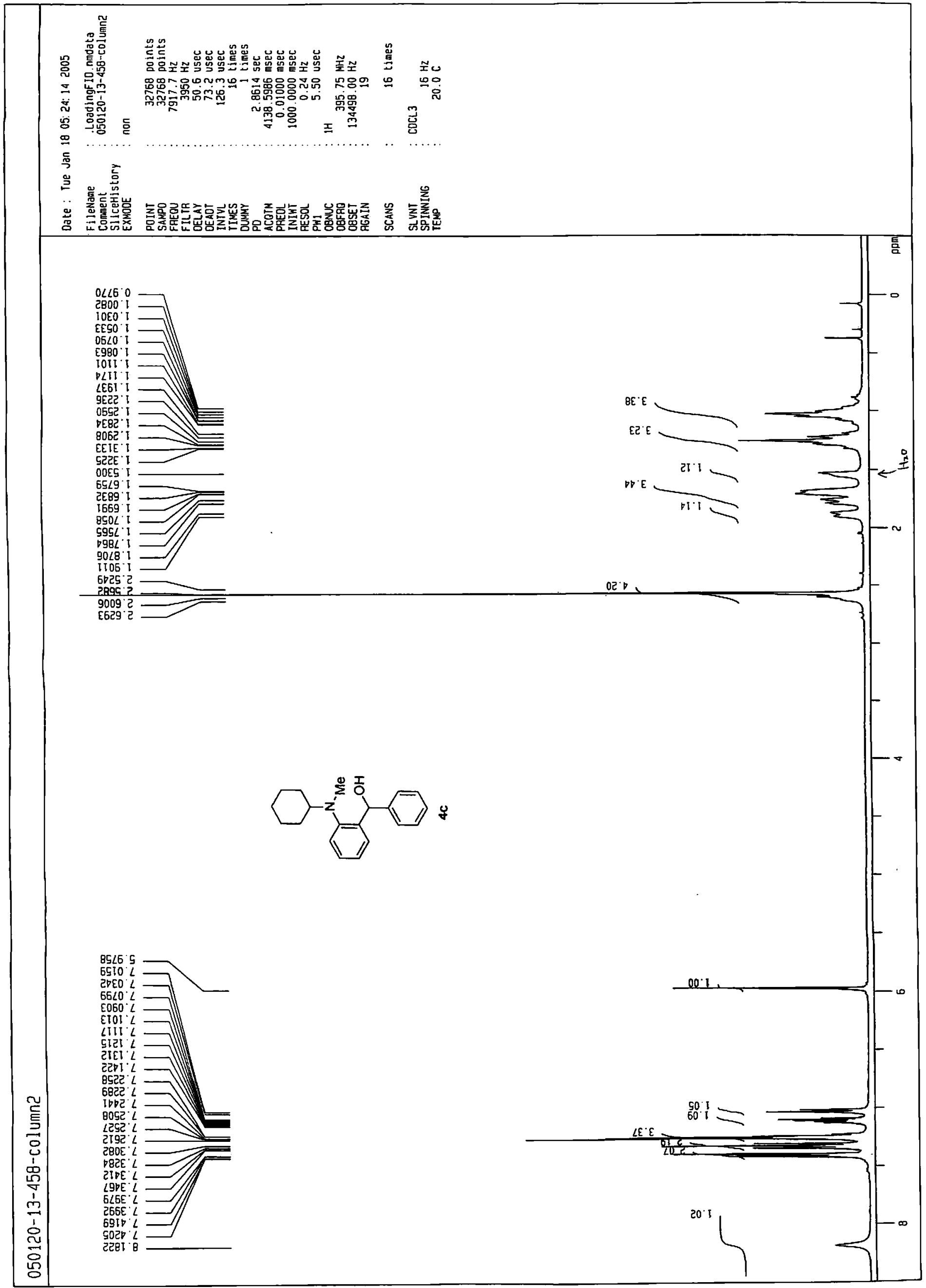




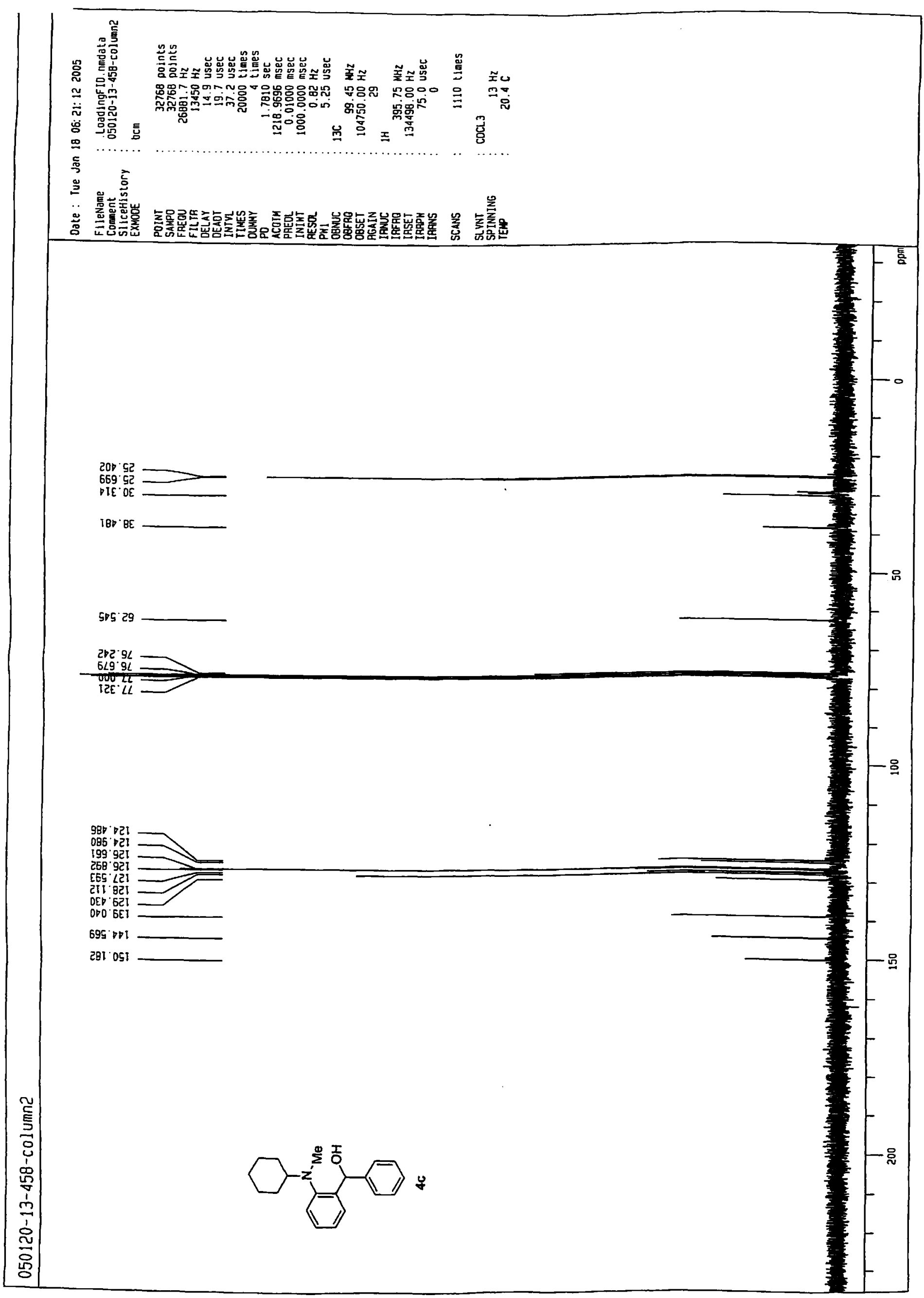




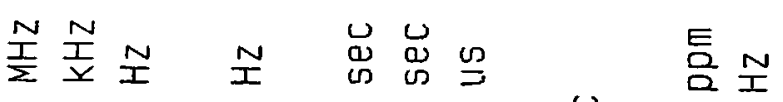

車

讪 六要品

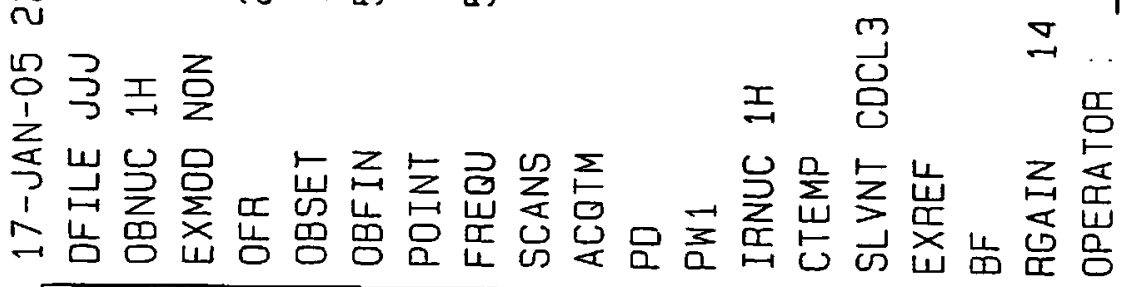

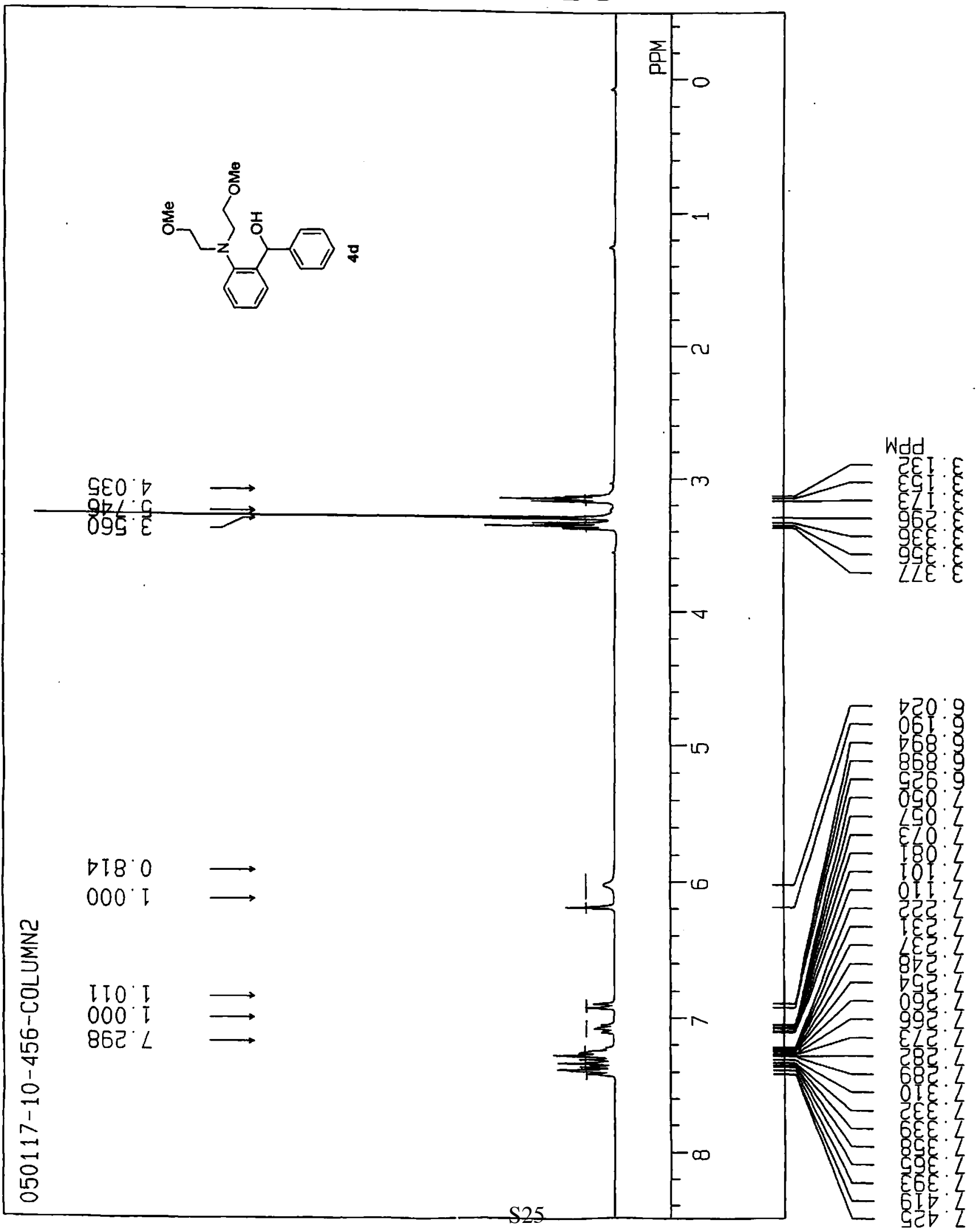




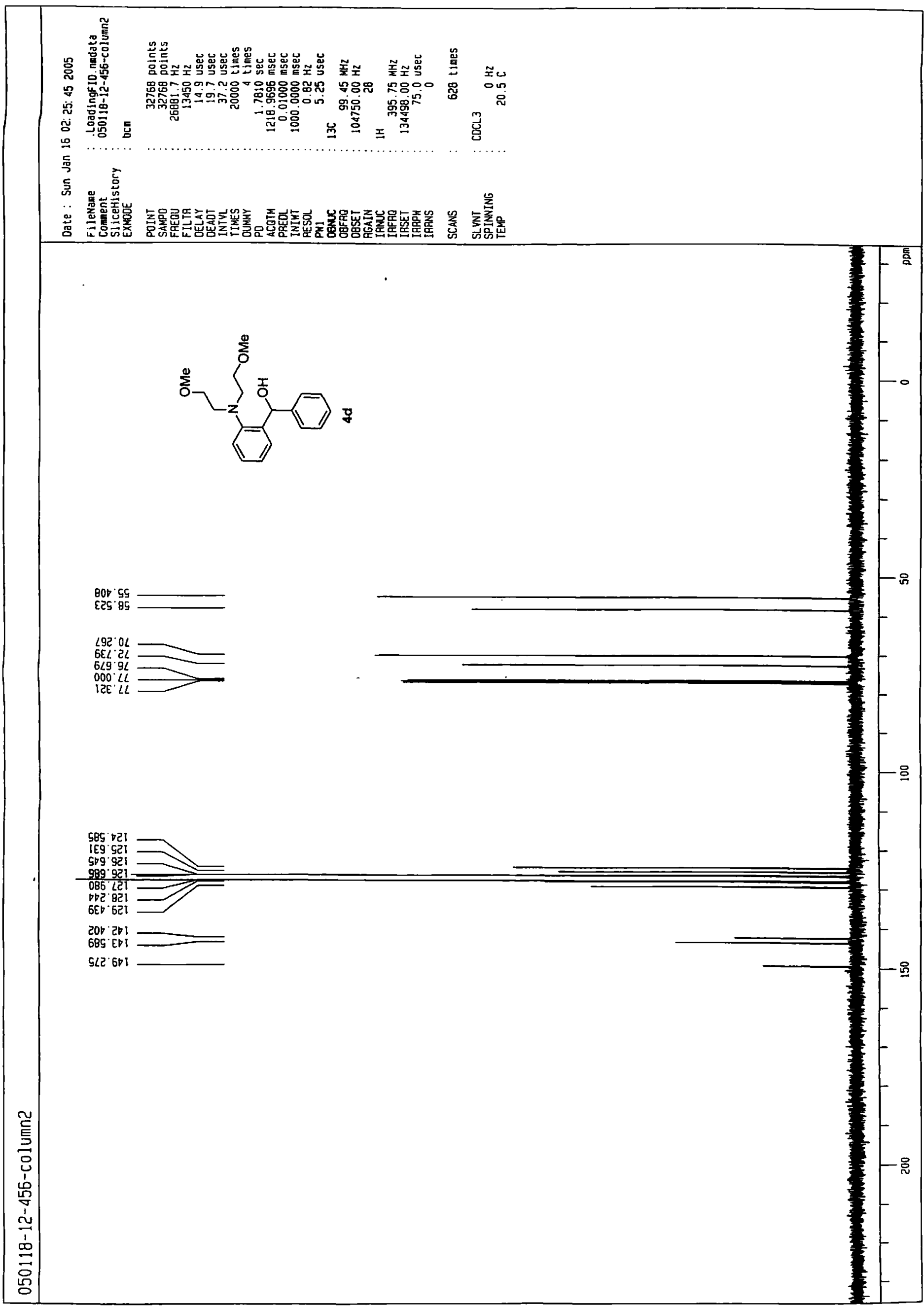




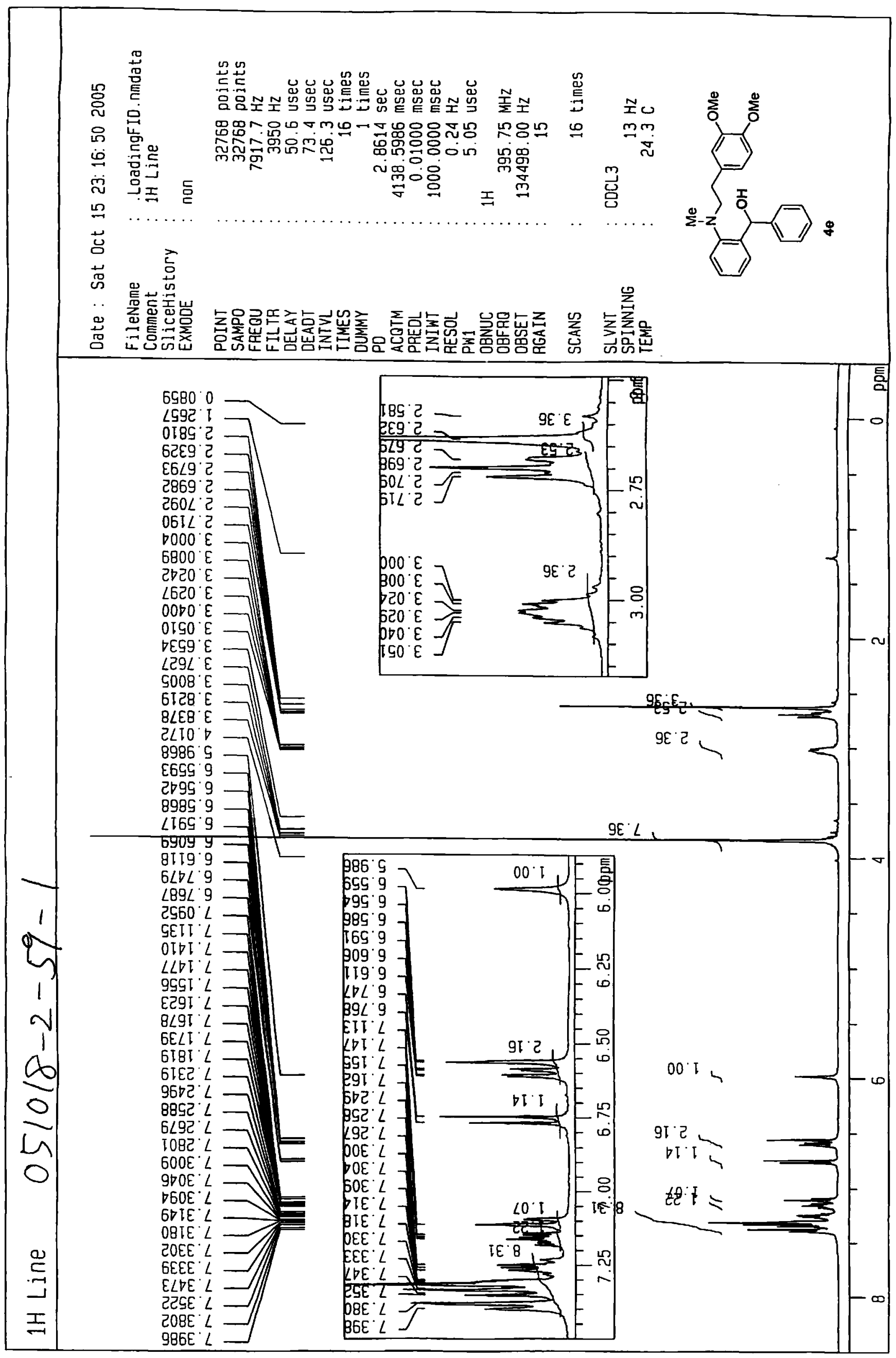




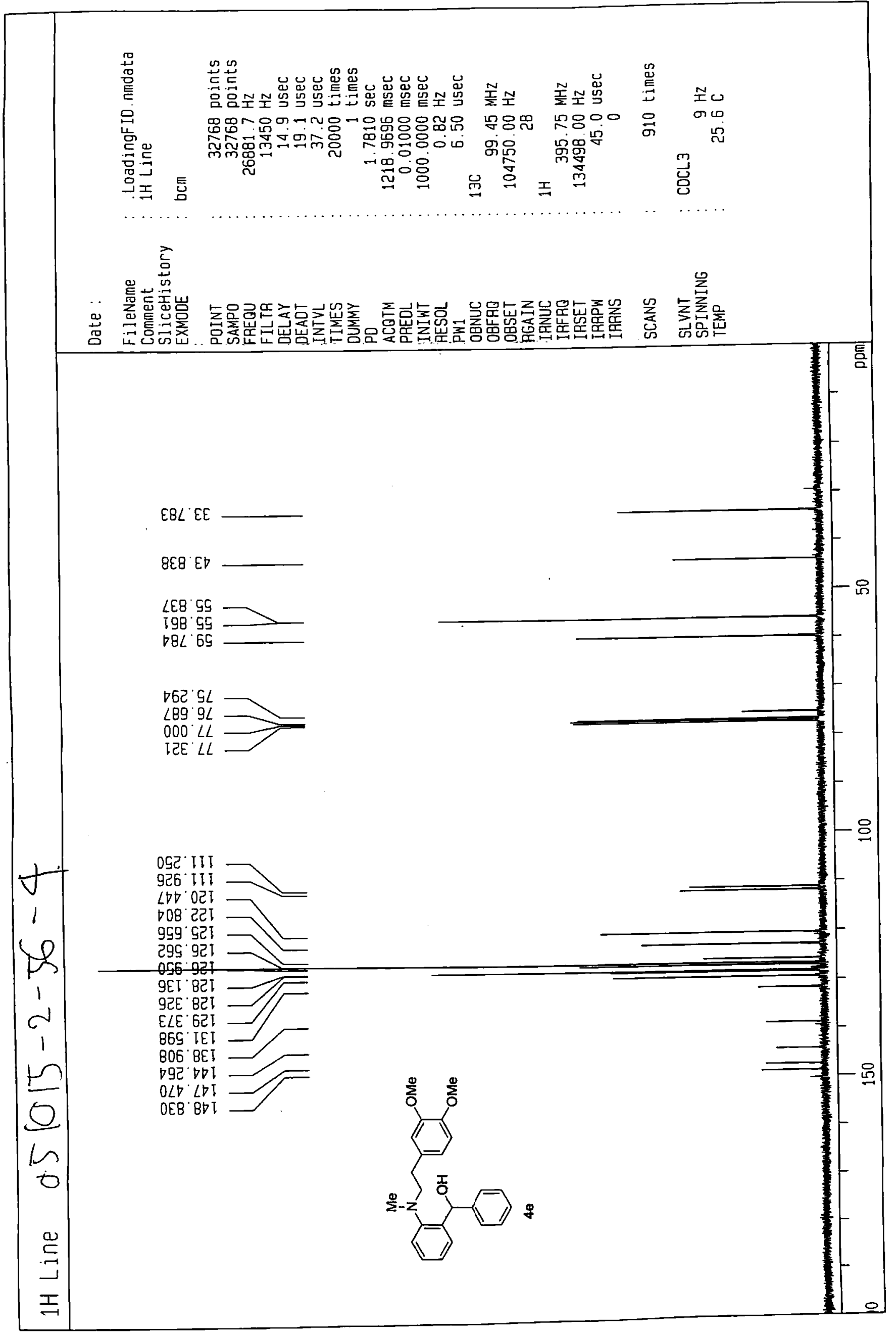




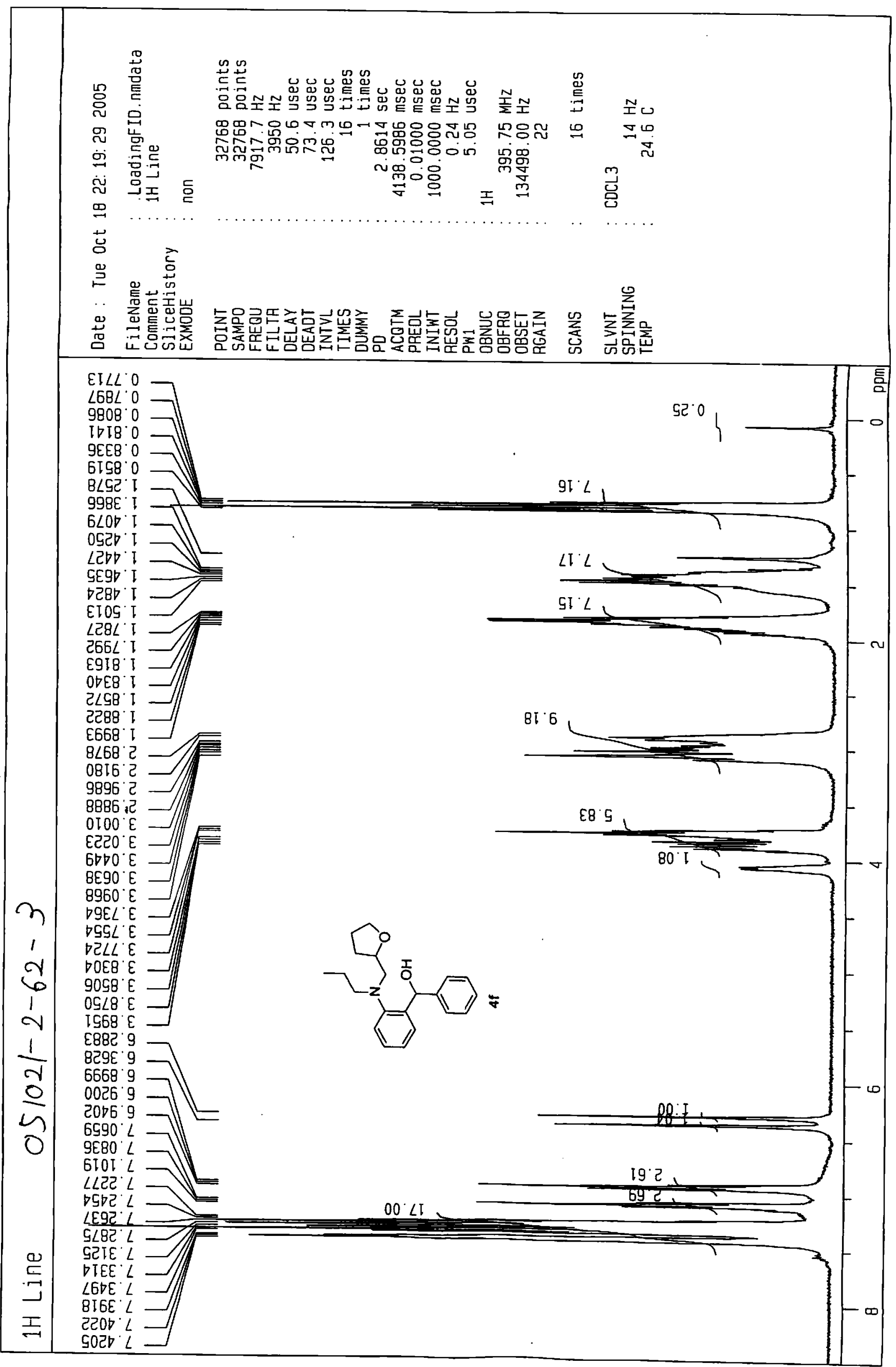




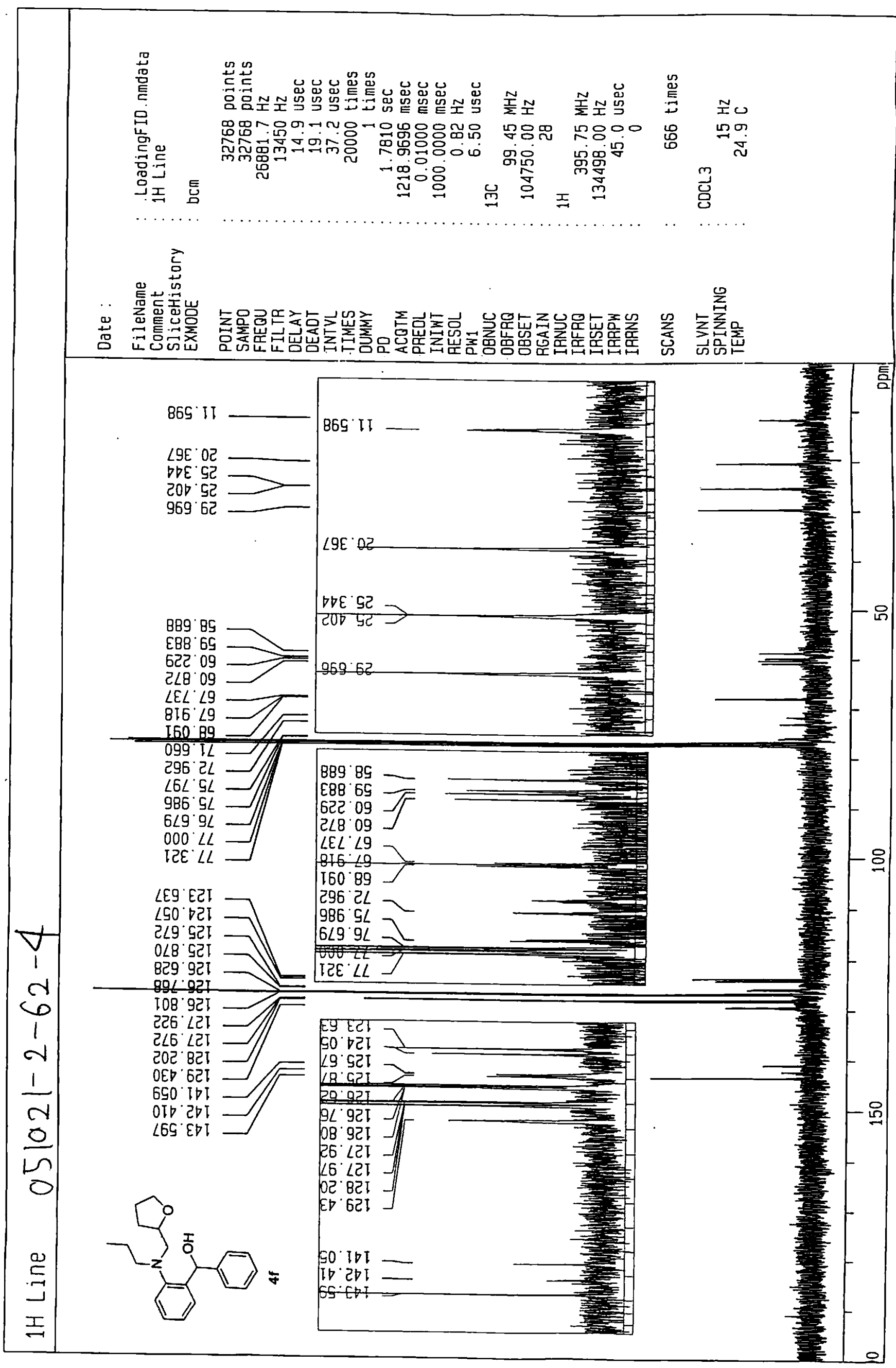




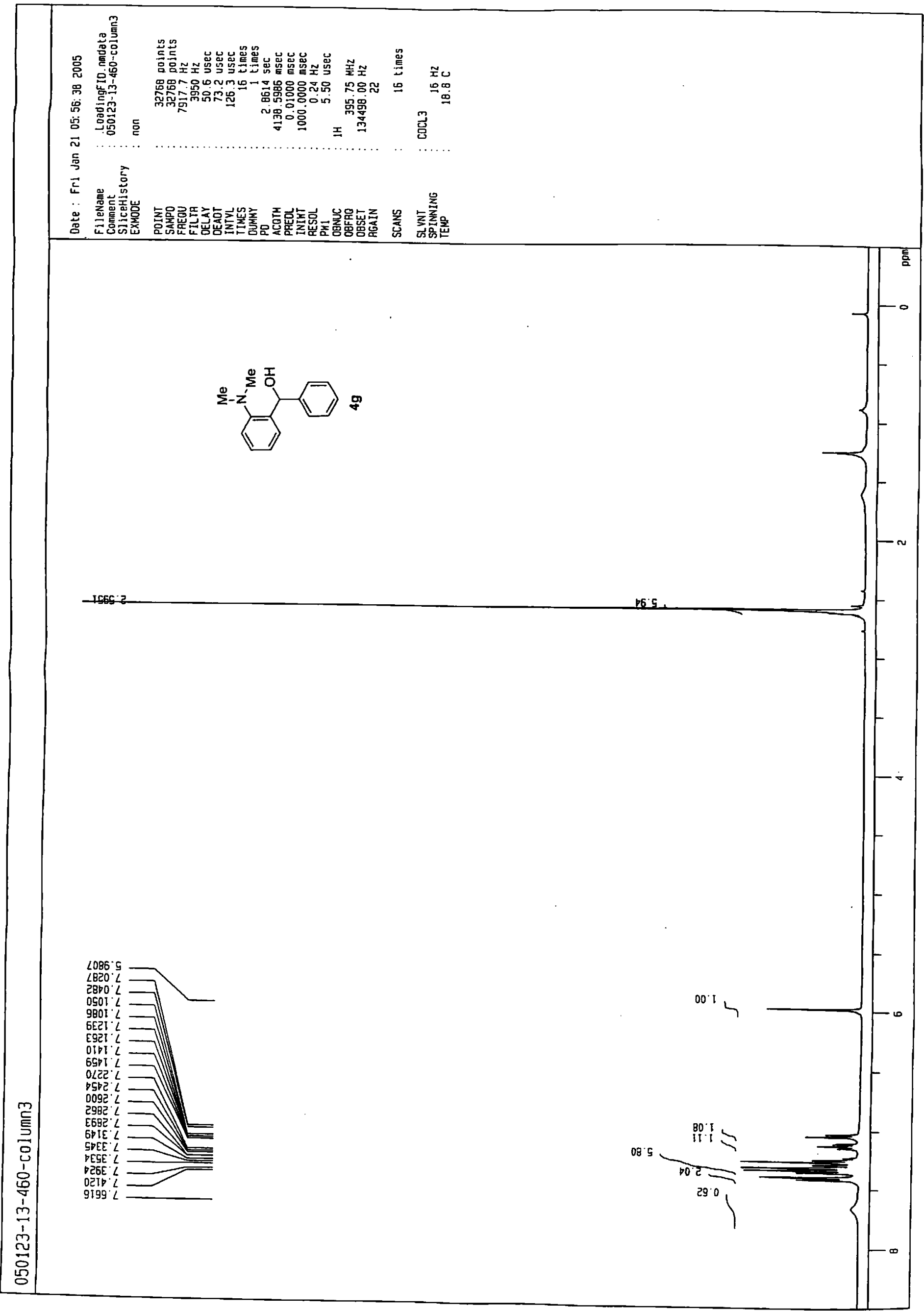




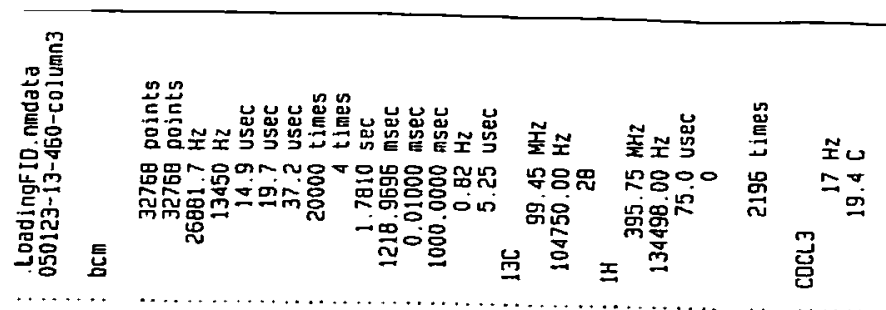

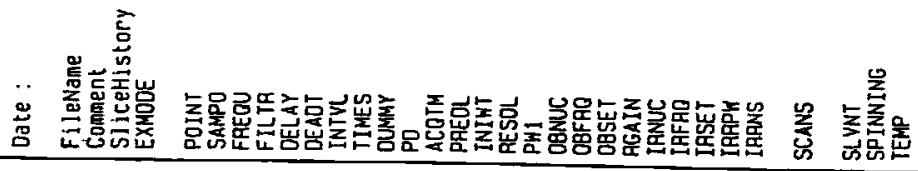

269.55

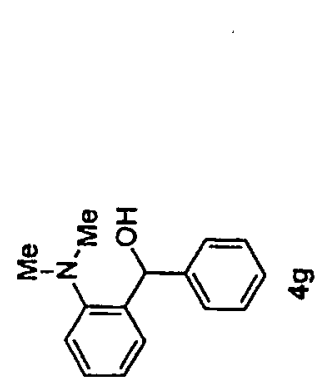

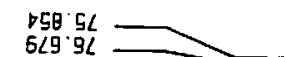

EIE $L L$

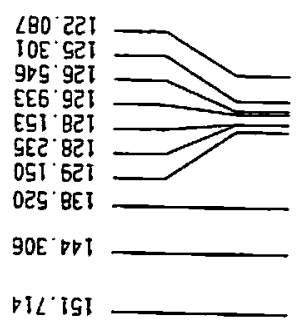


m

i்

$\dot{8}$

0

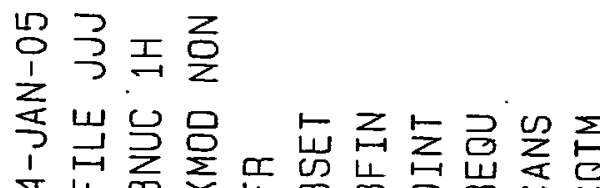

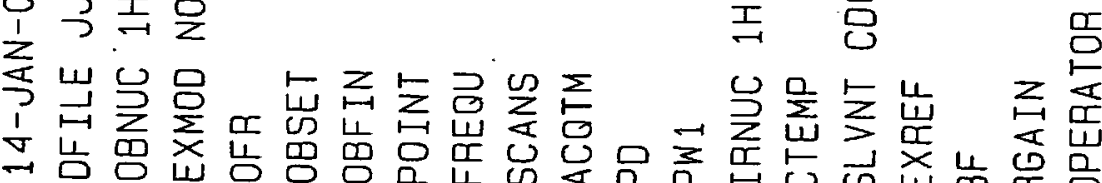
மீ요유

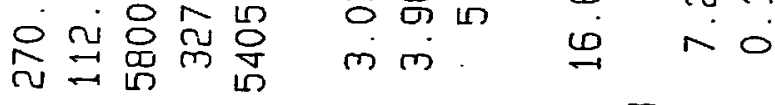

工总

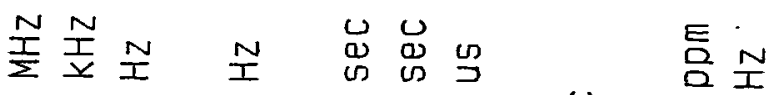

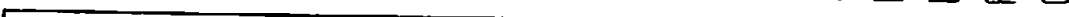

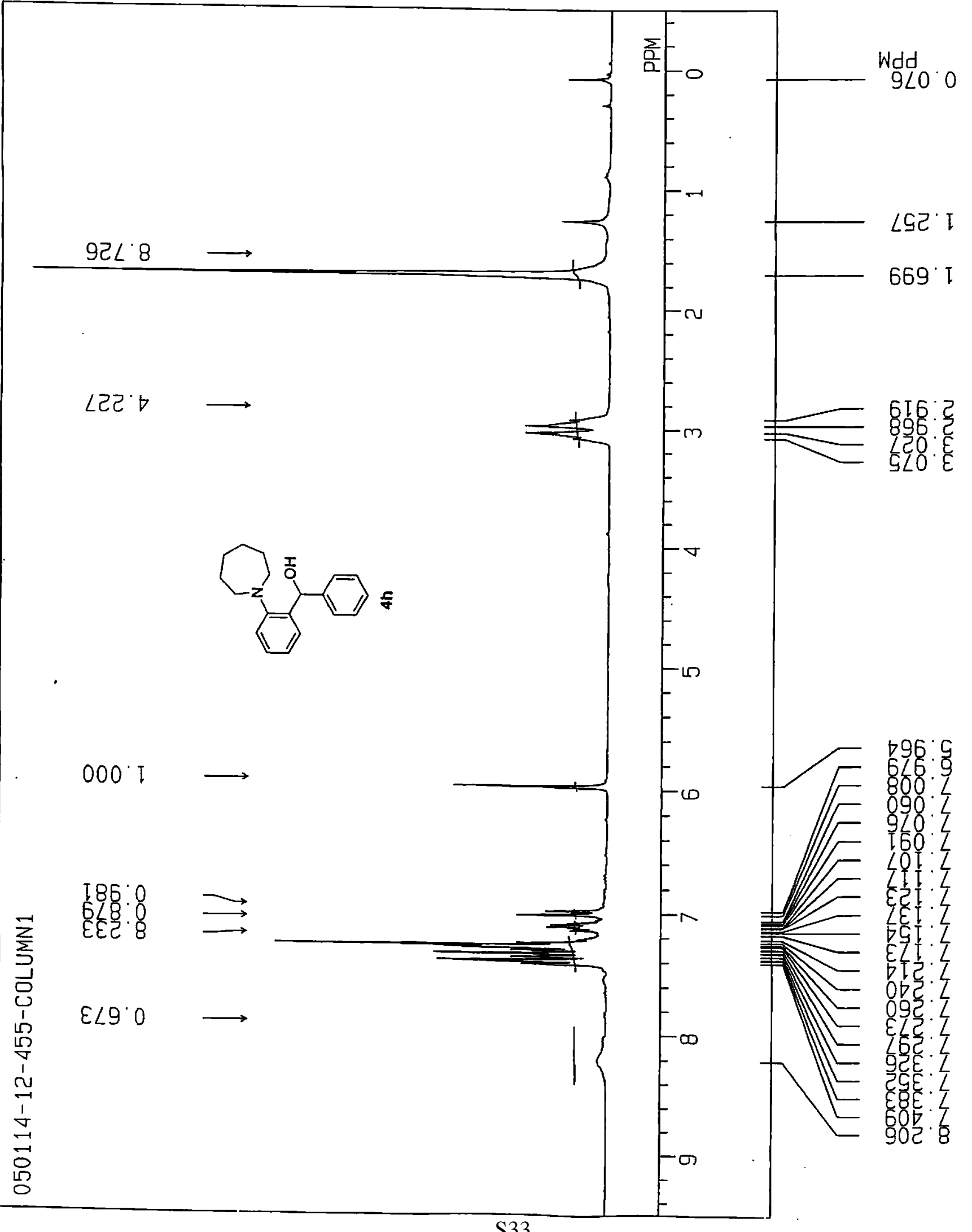




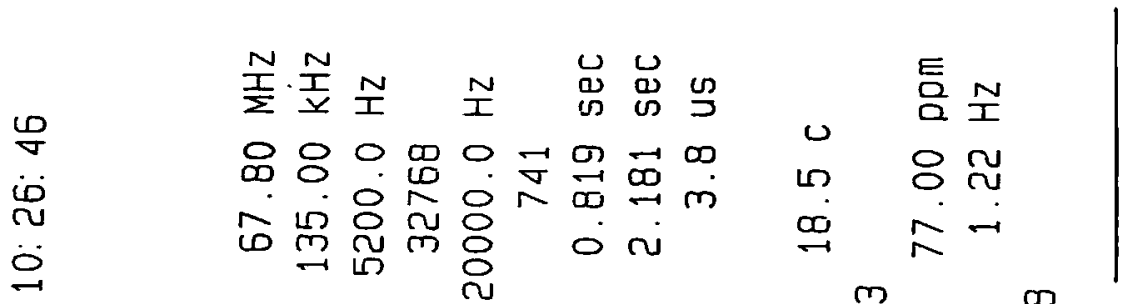

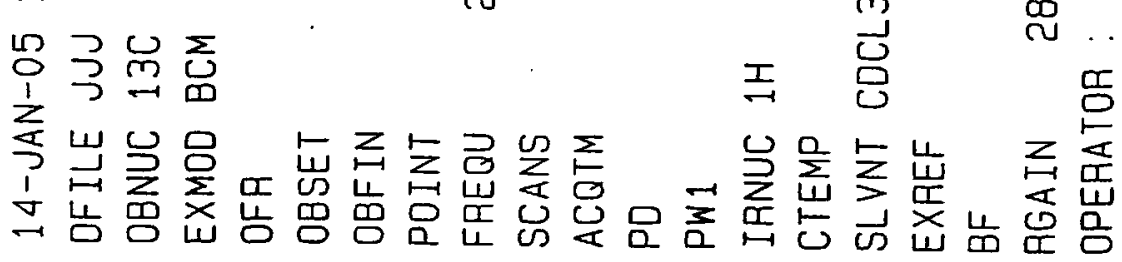

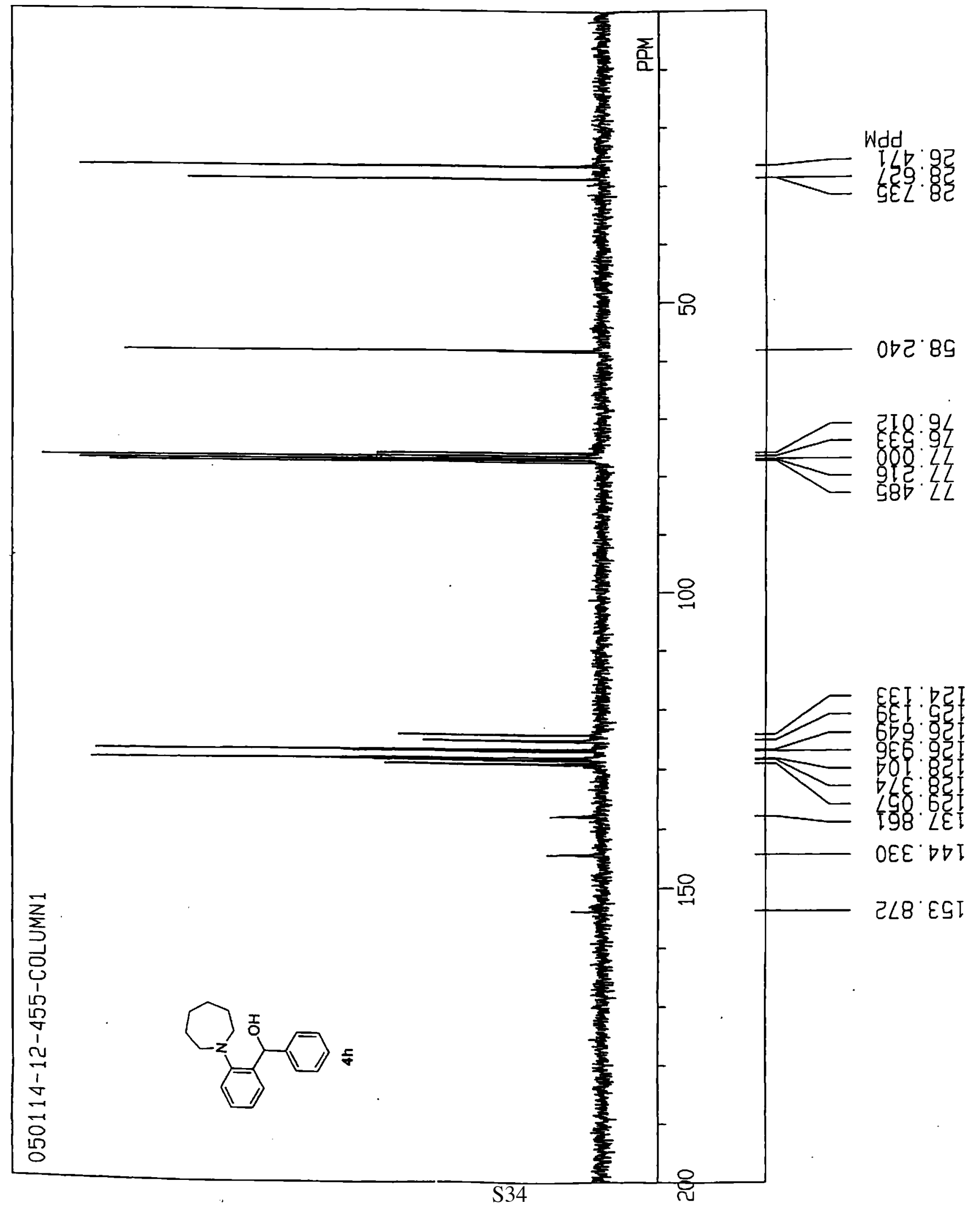


in

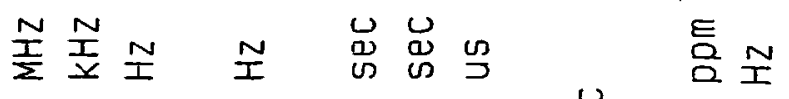

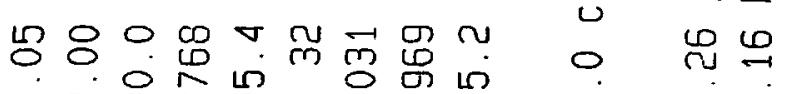

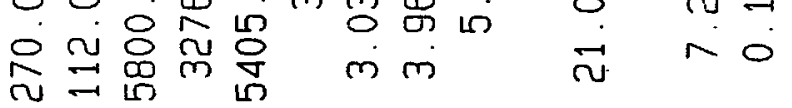

$\ddot{g}$

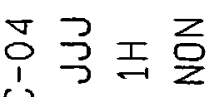

工 $\stackrel{m}{\overrightarrow{0}}$

$\stackrel{\infty}{\sim}$

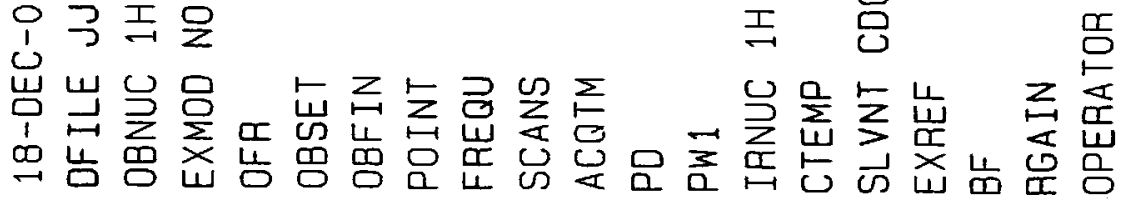

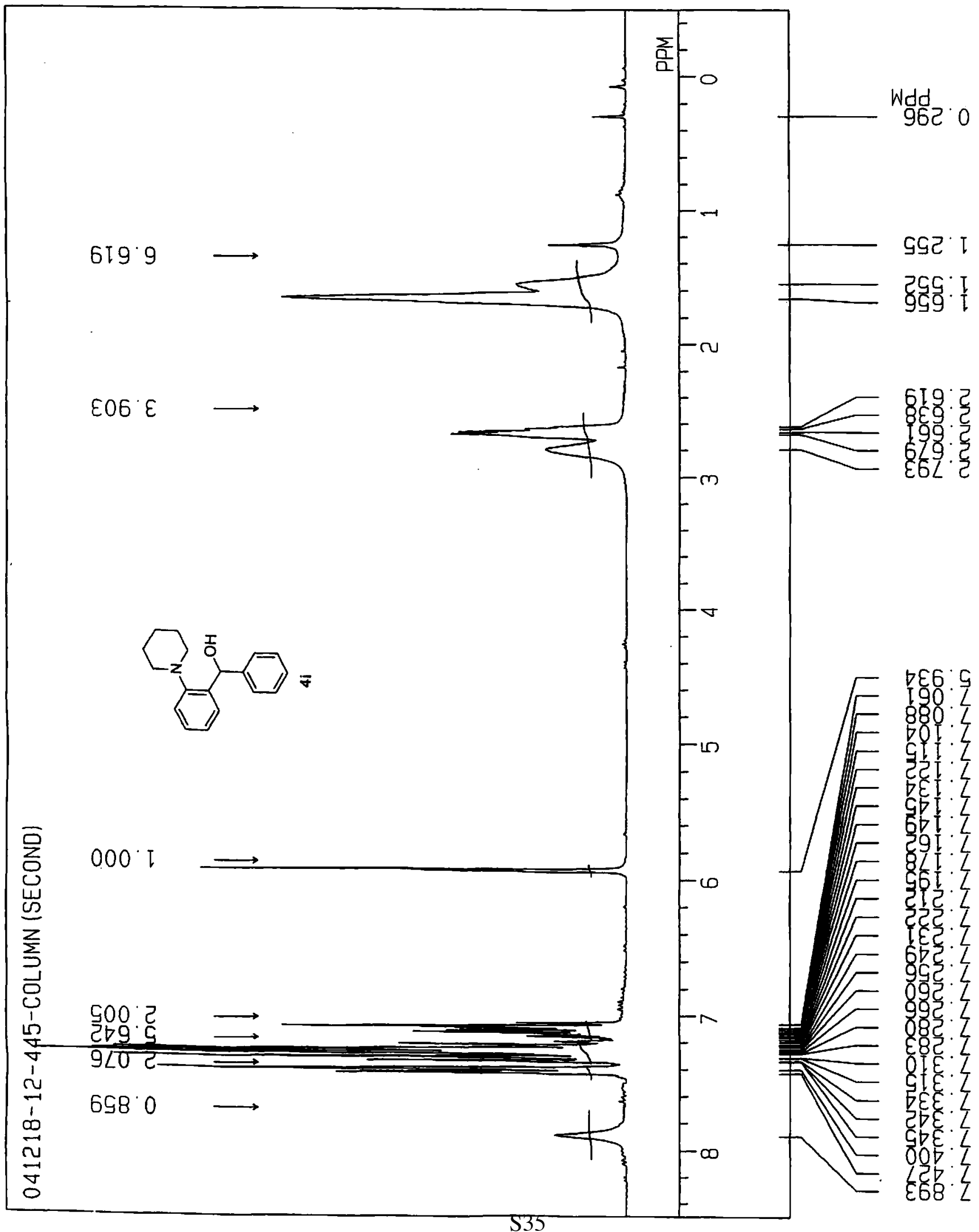




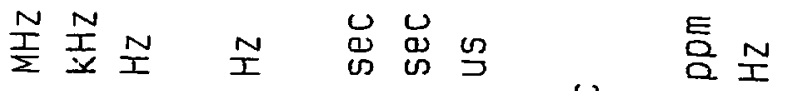

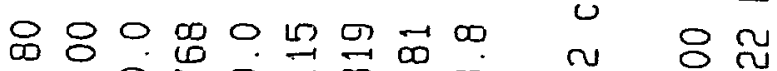

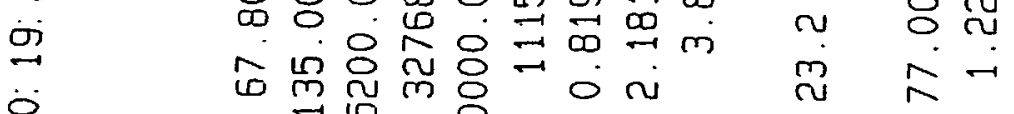

蚛

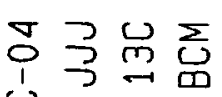

I

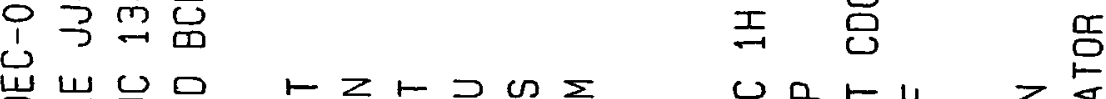

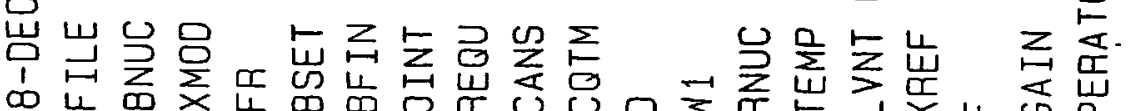

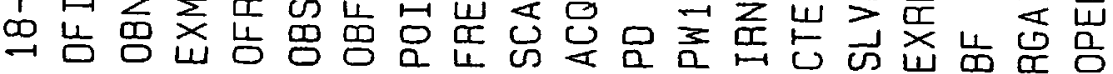

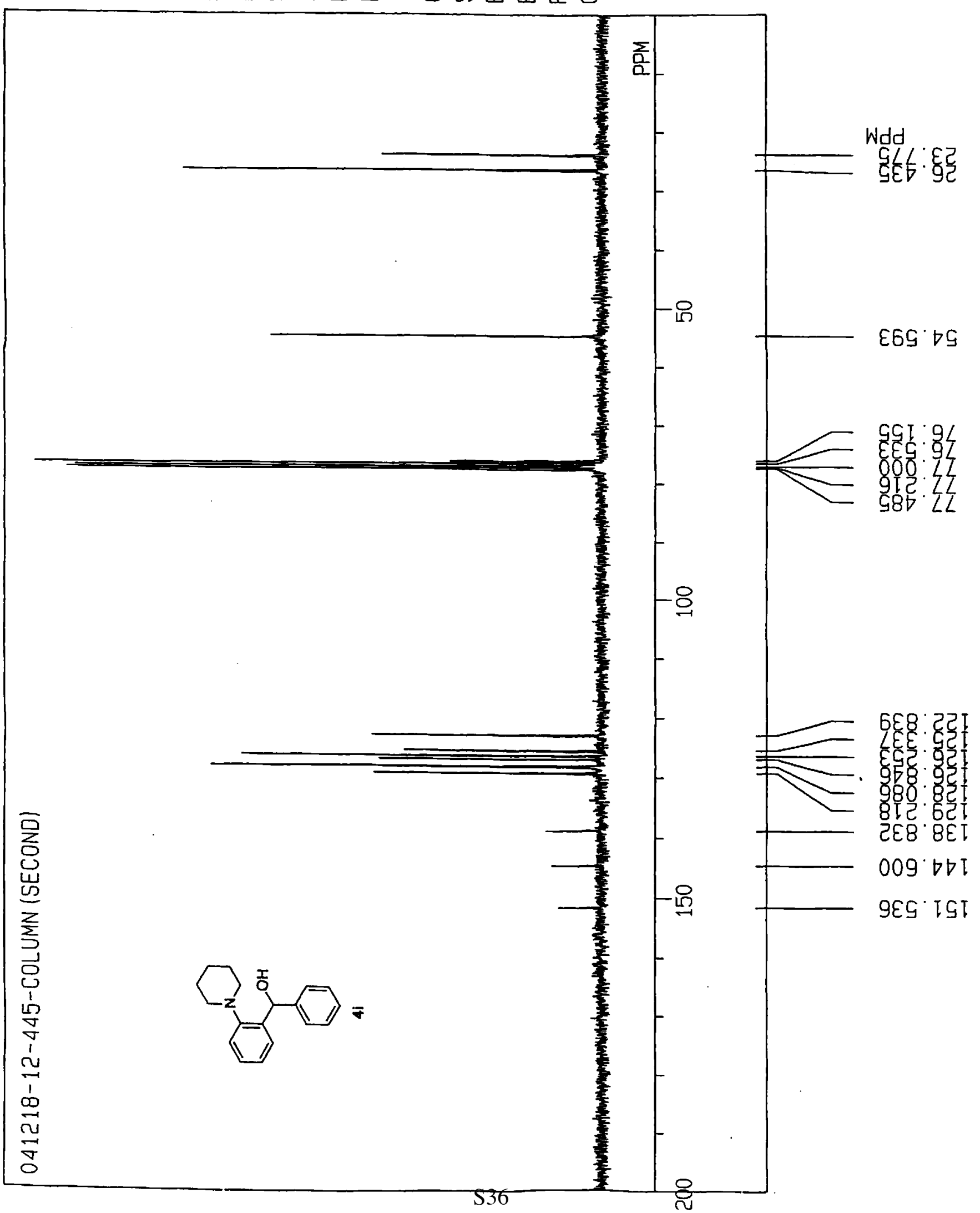




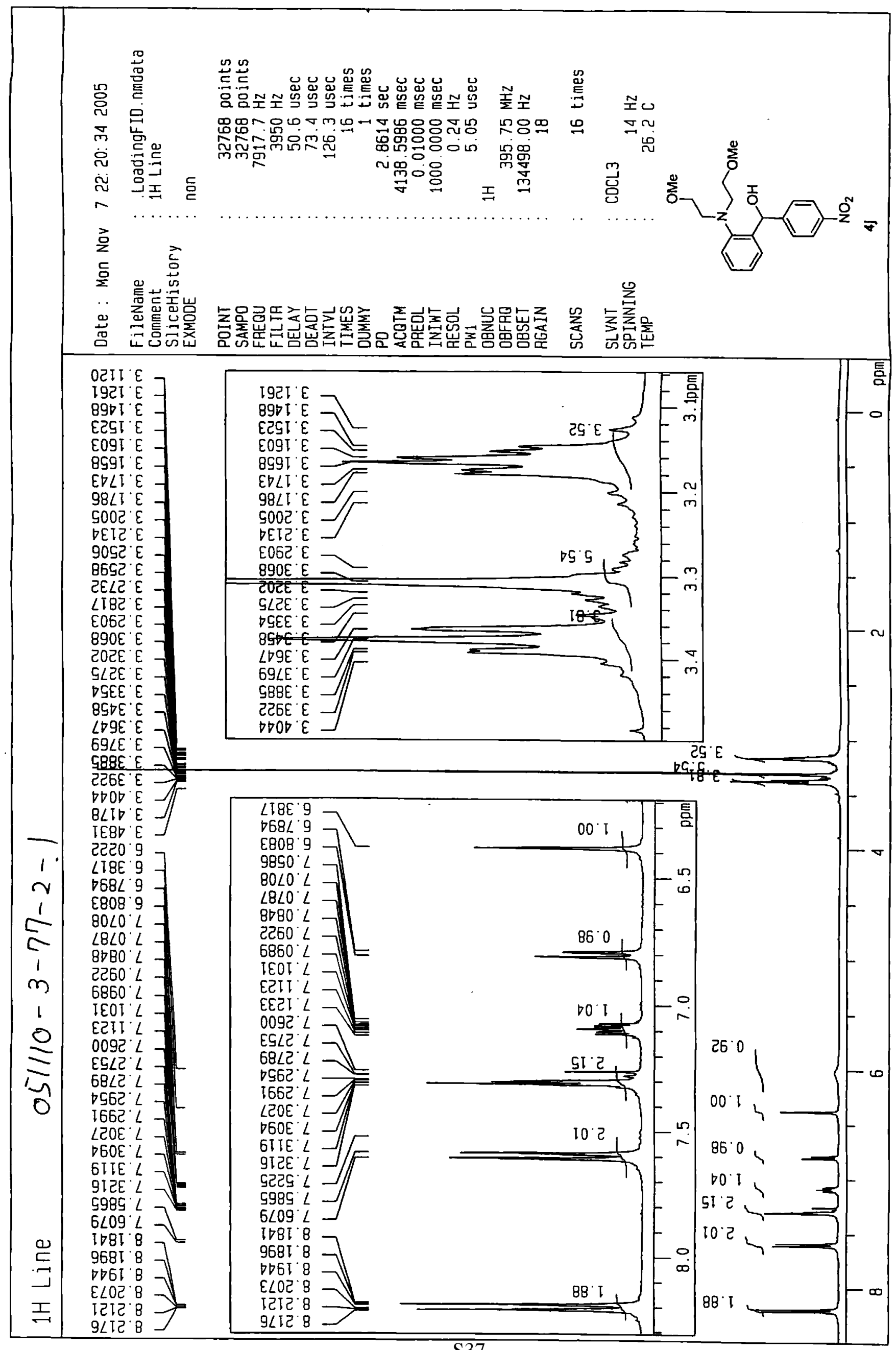




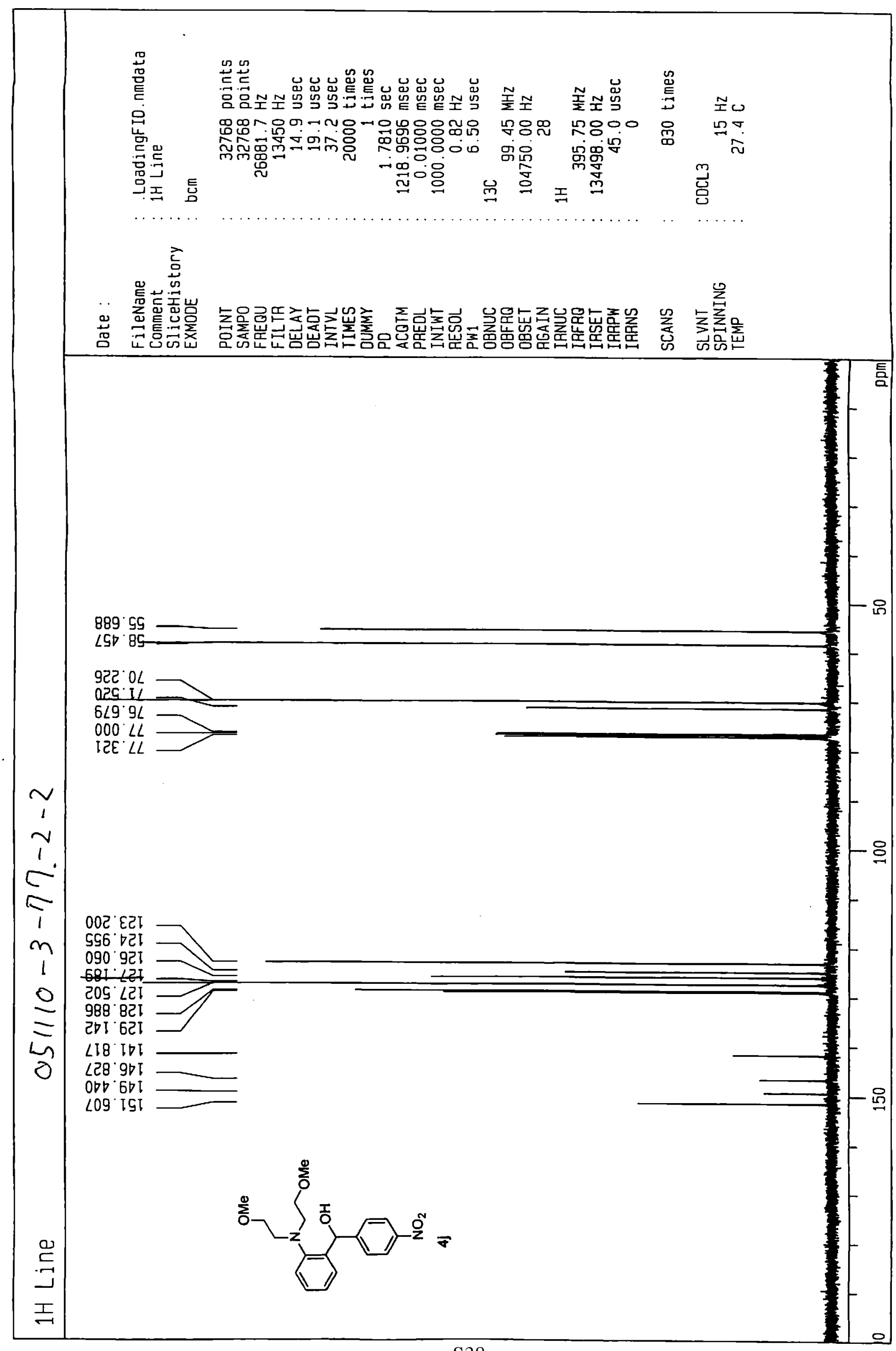




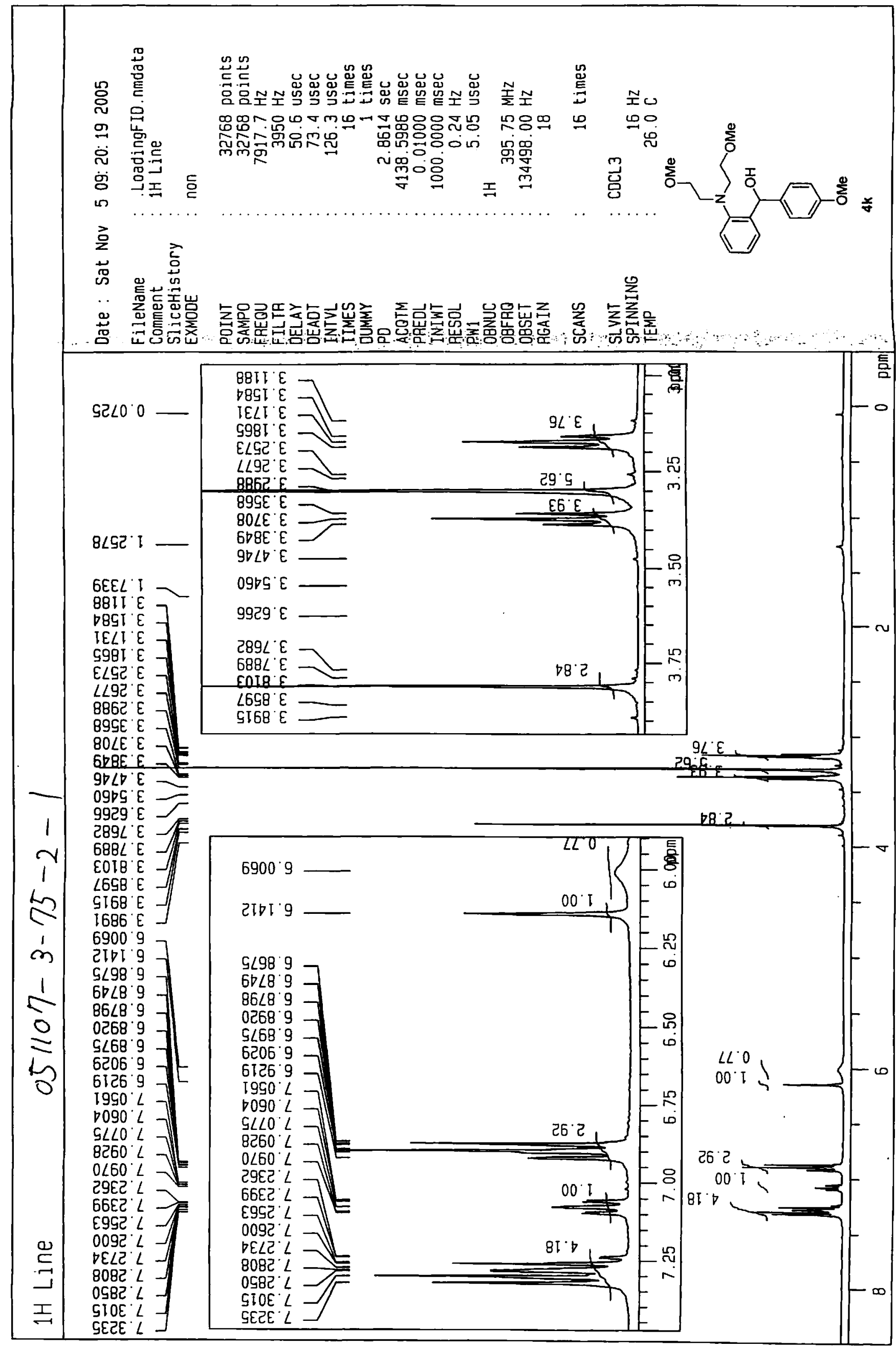




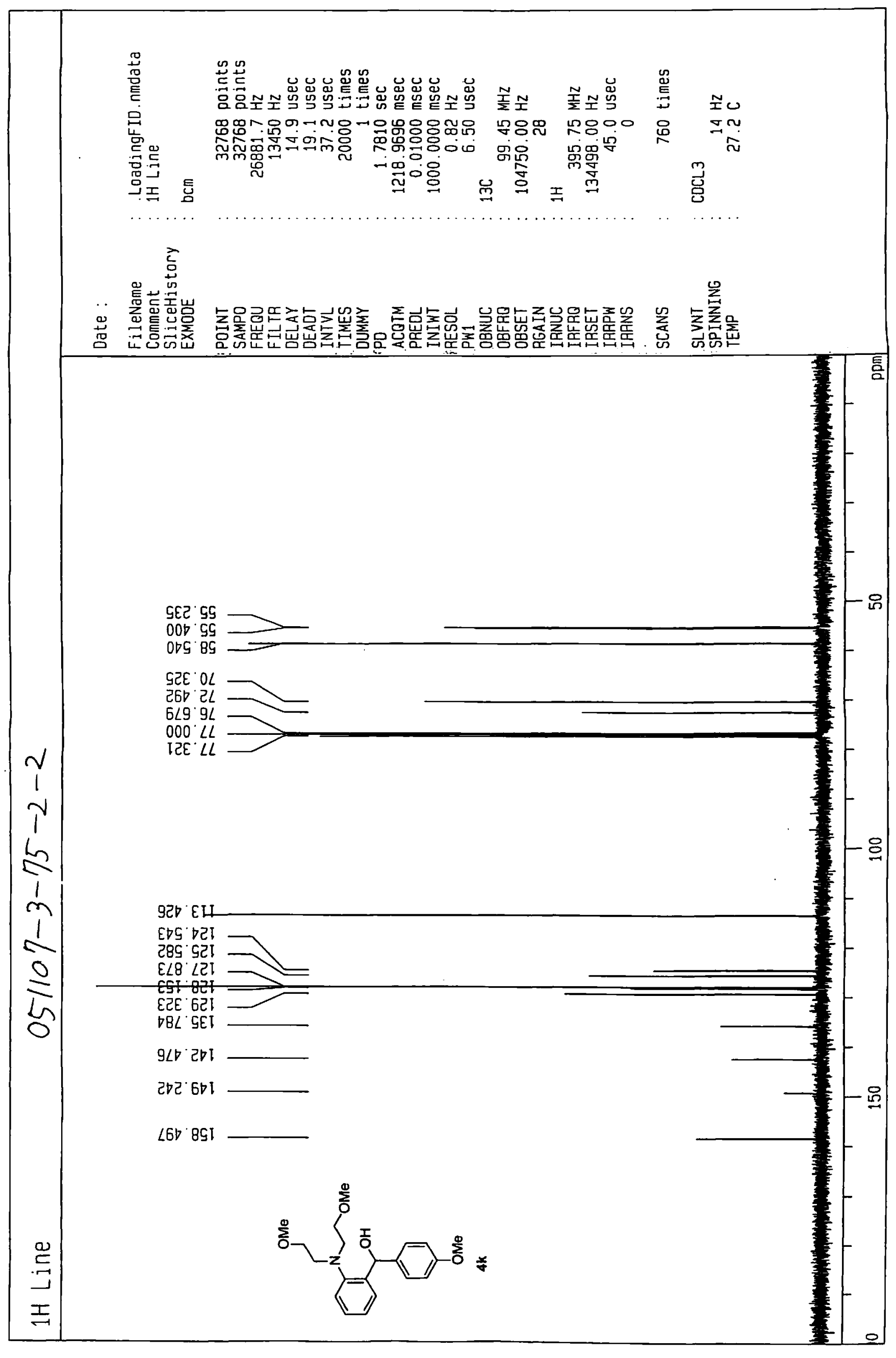




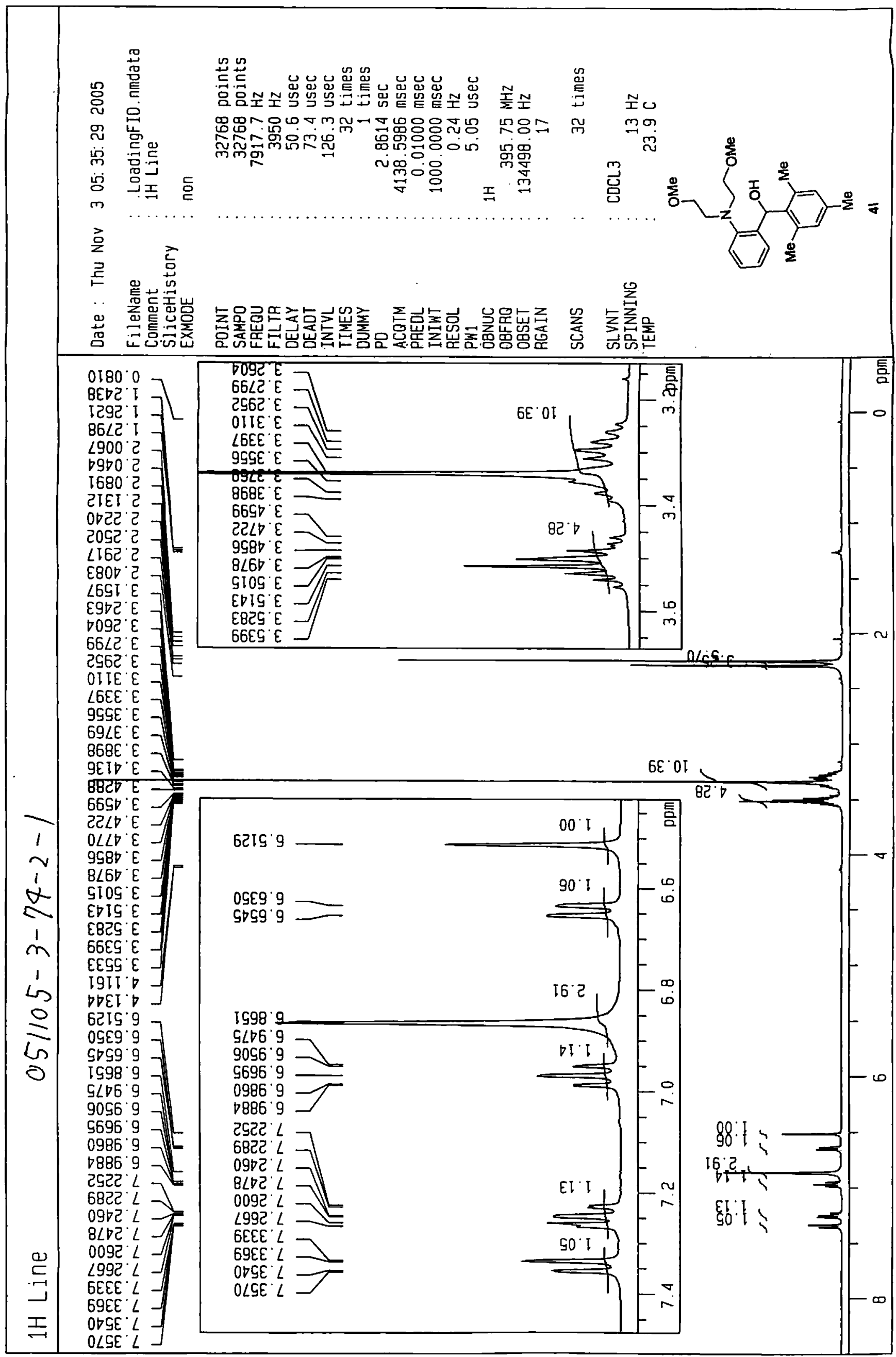




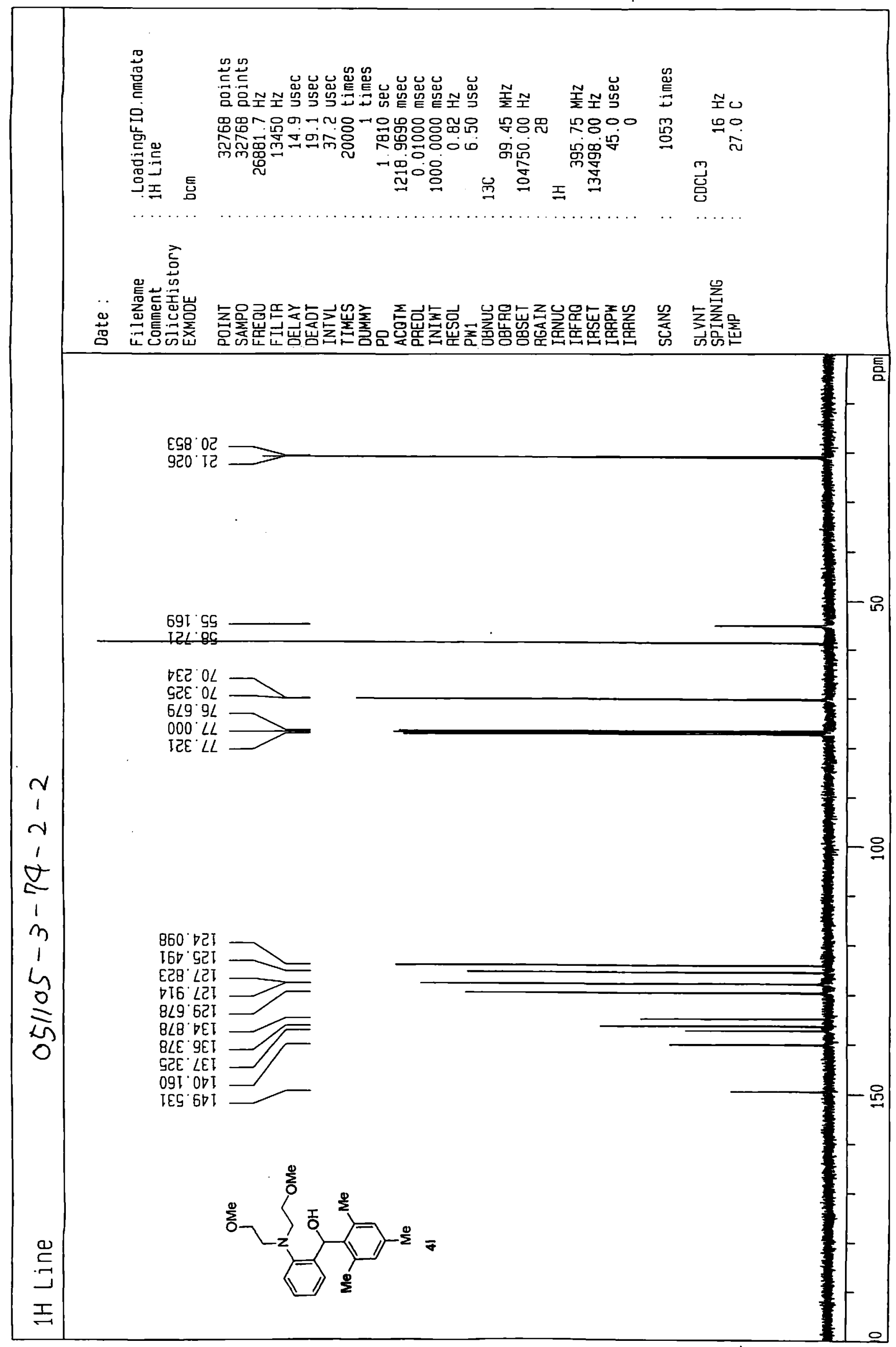




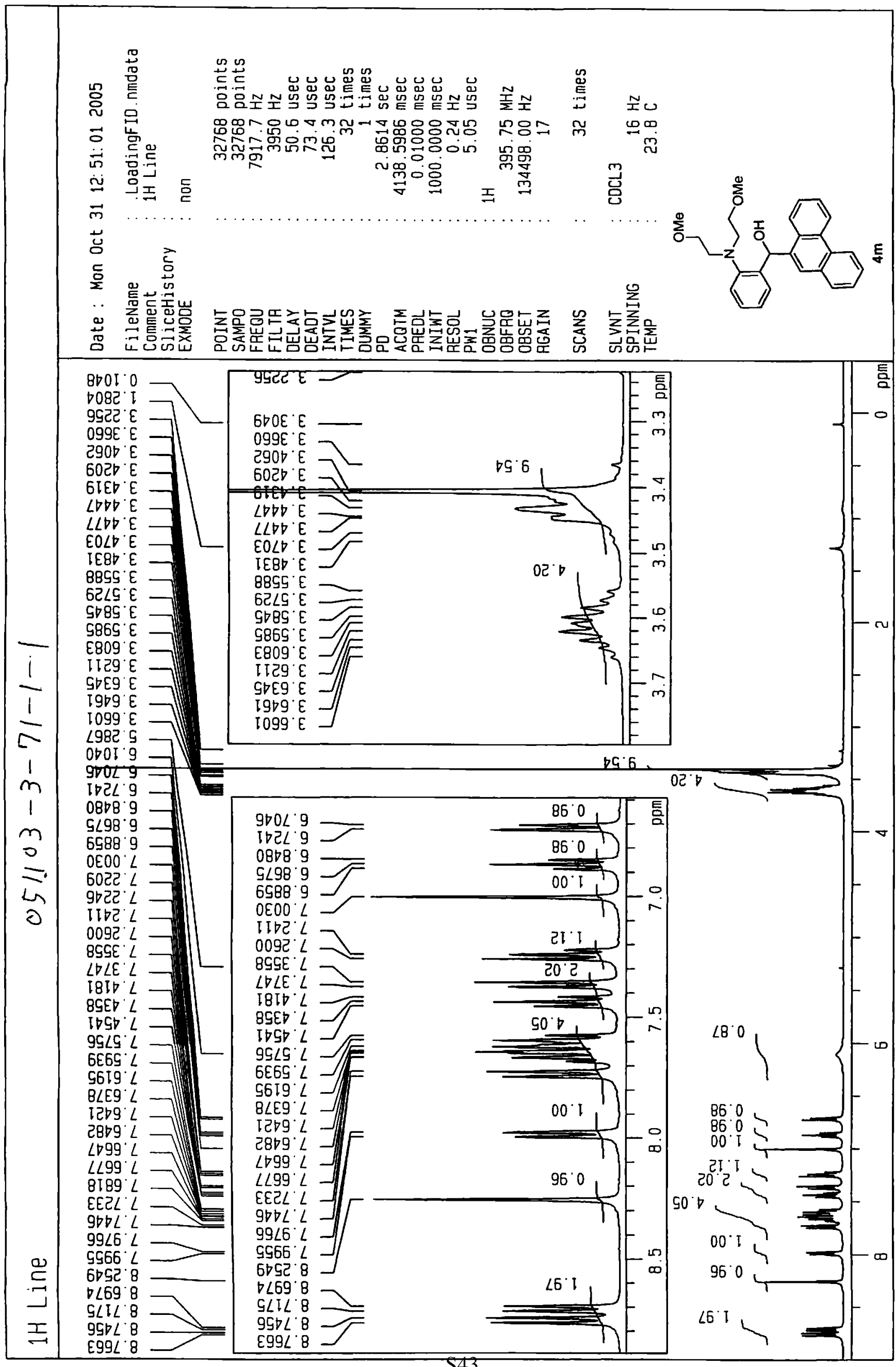




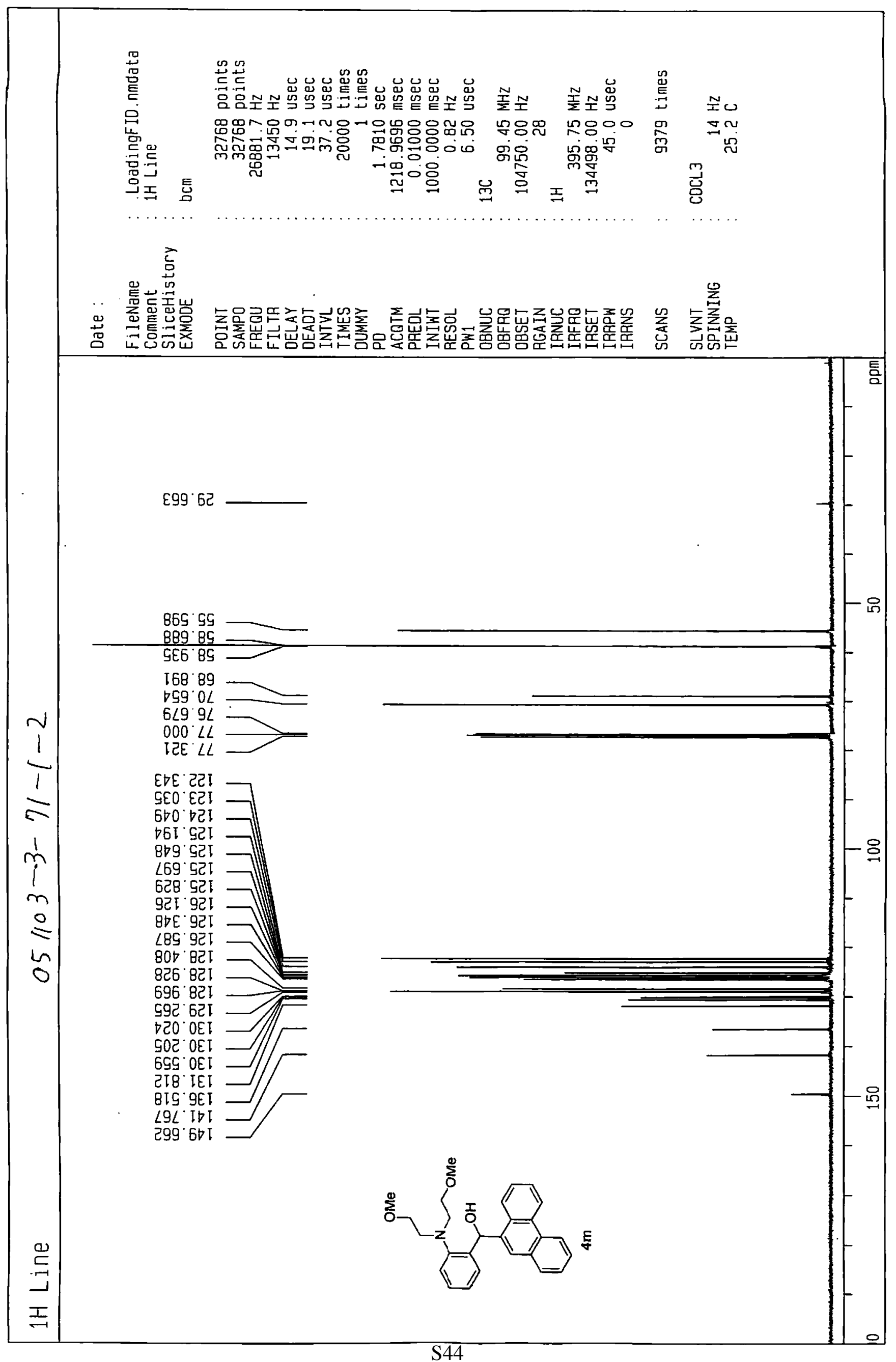




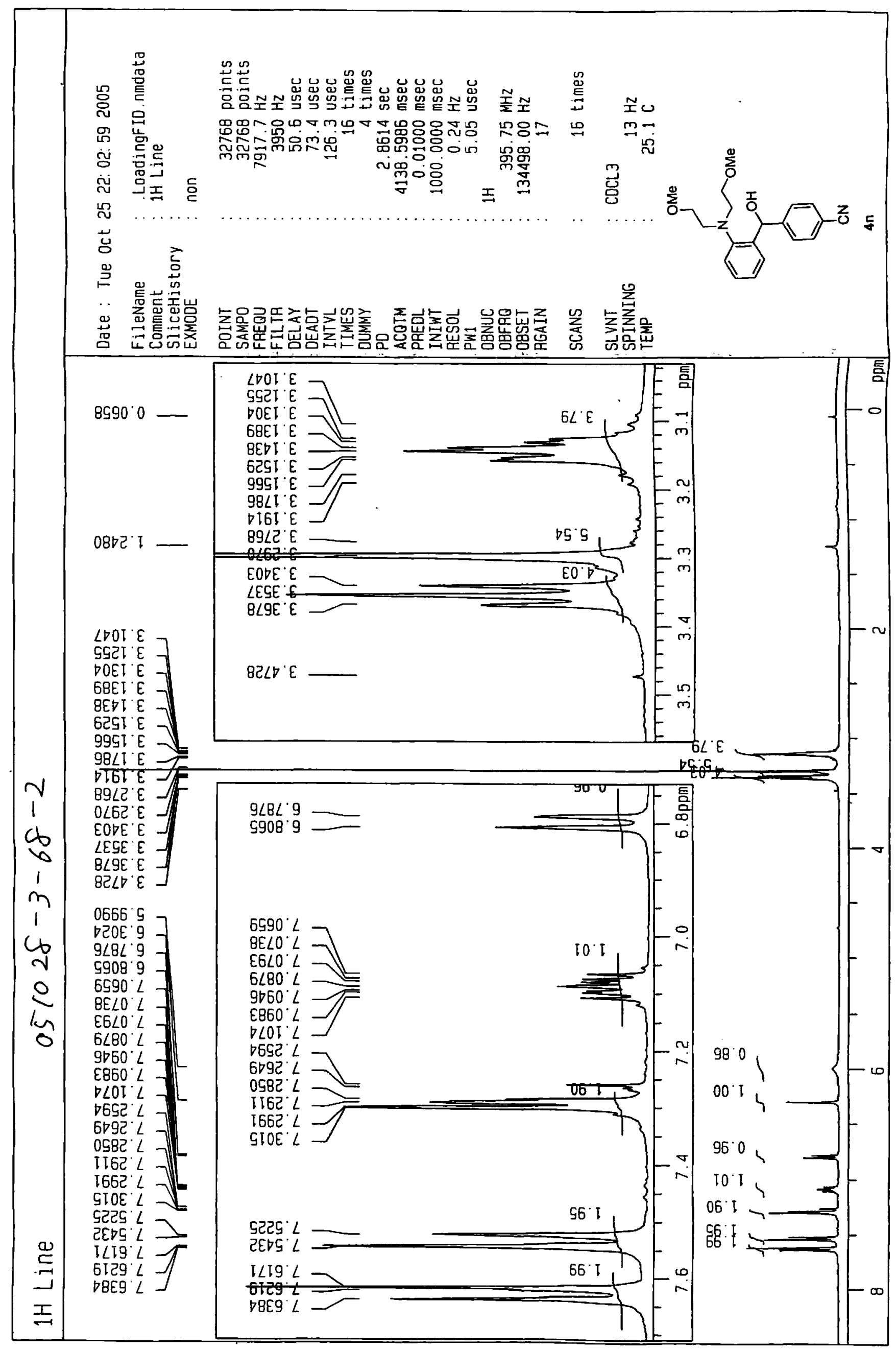




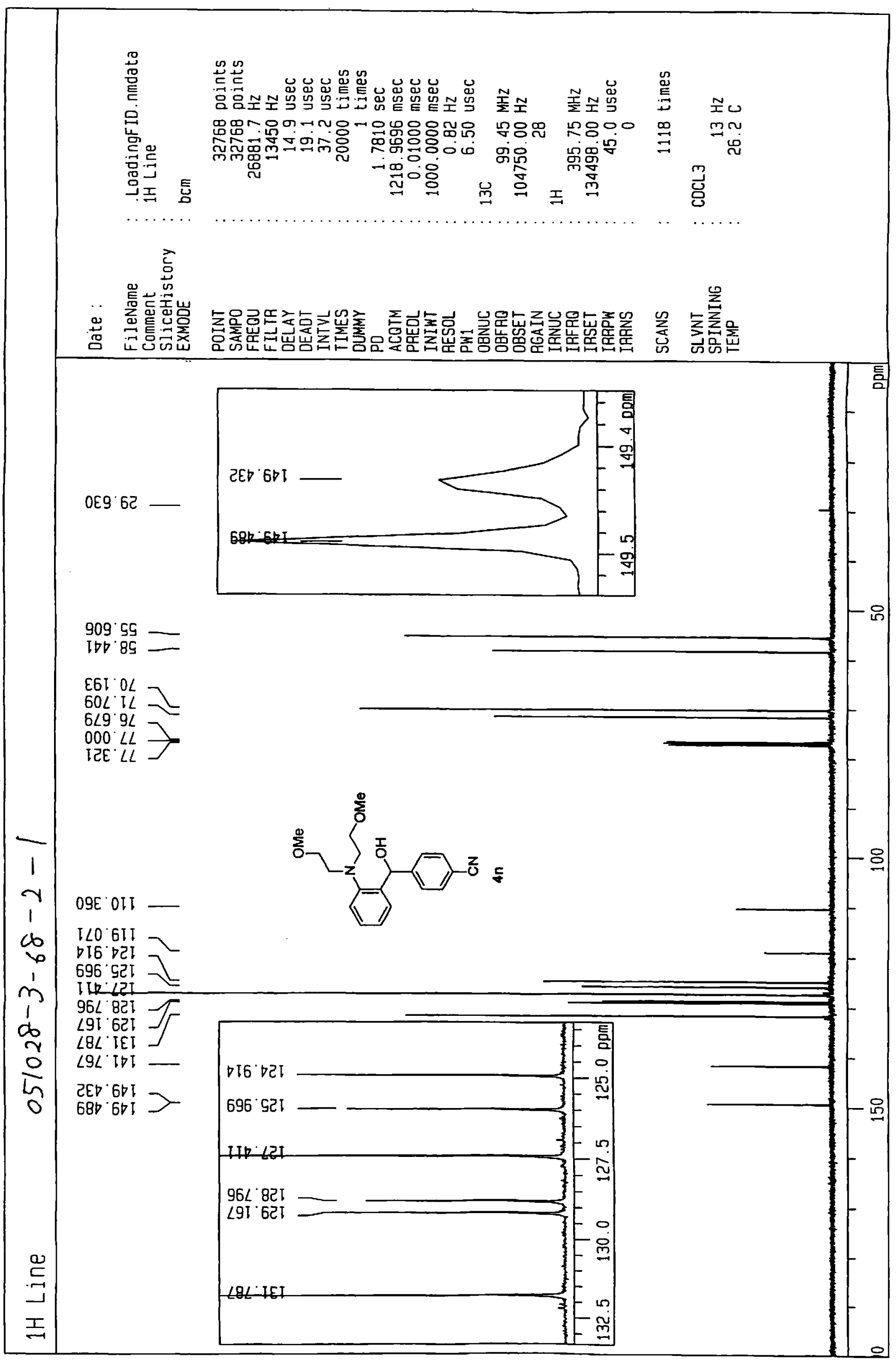




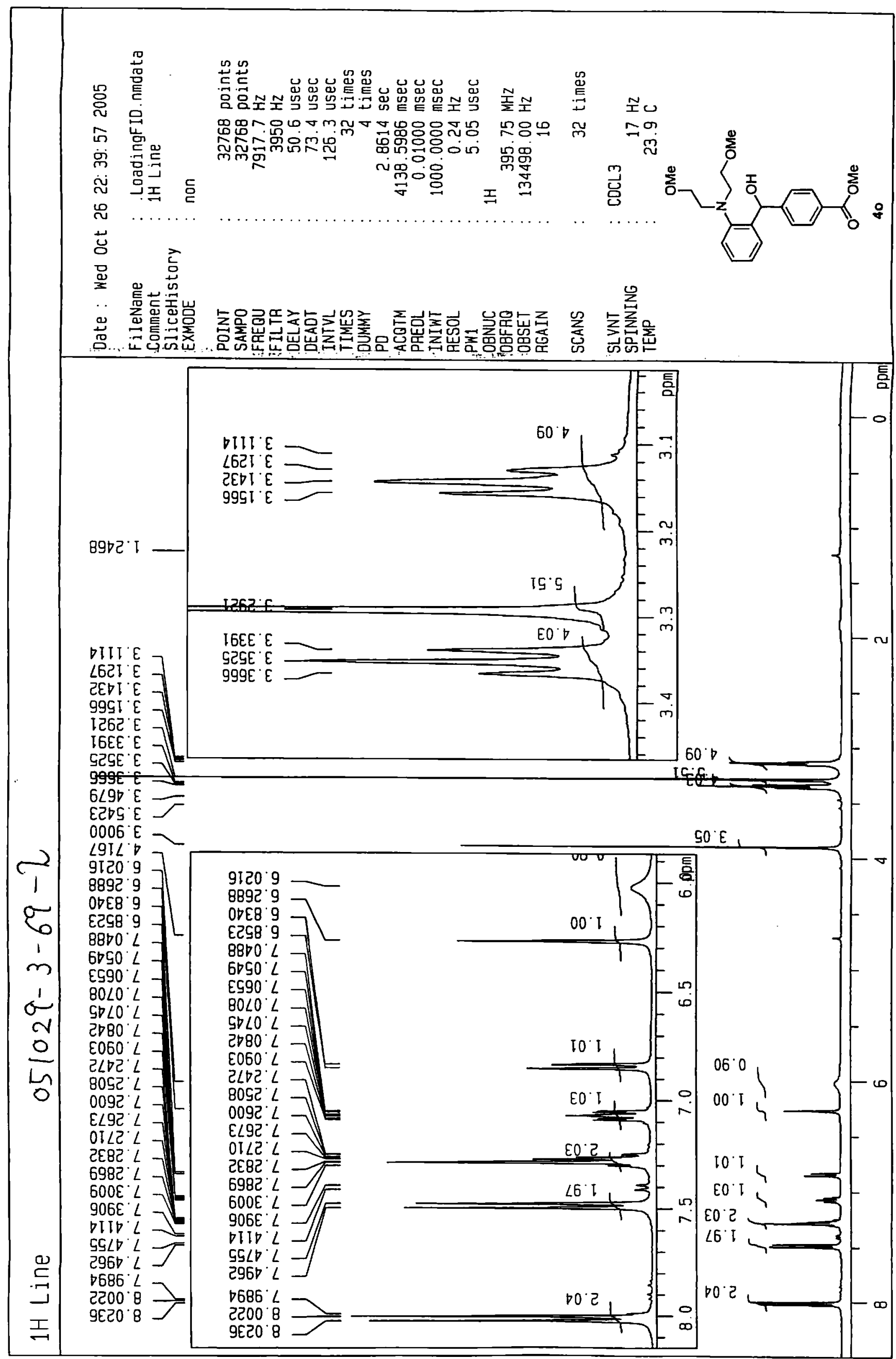




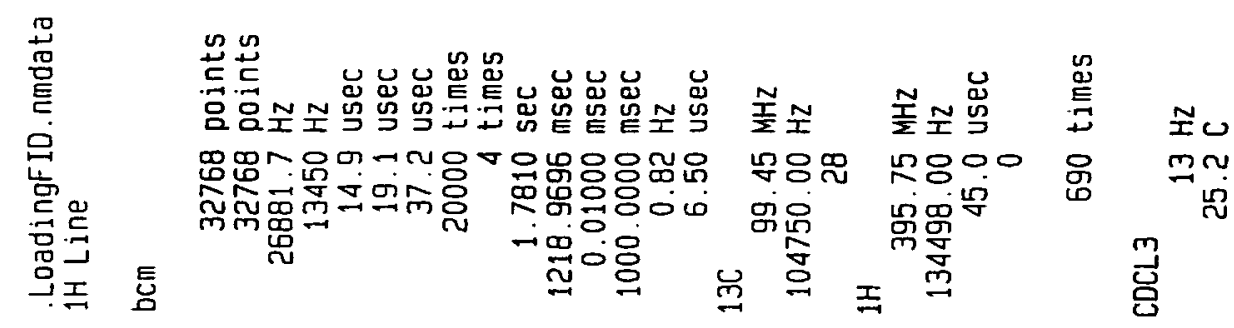

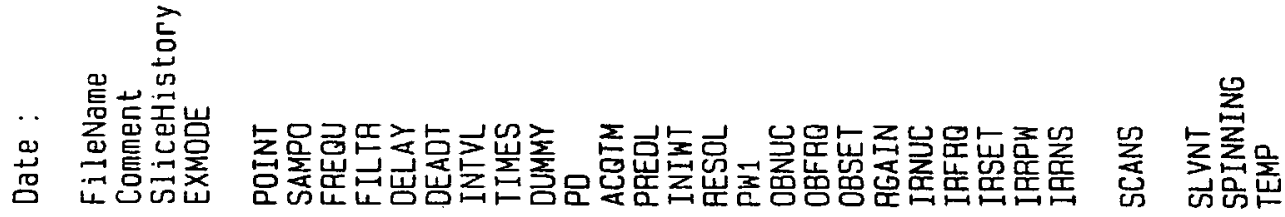

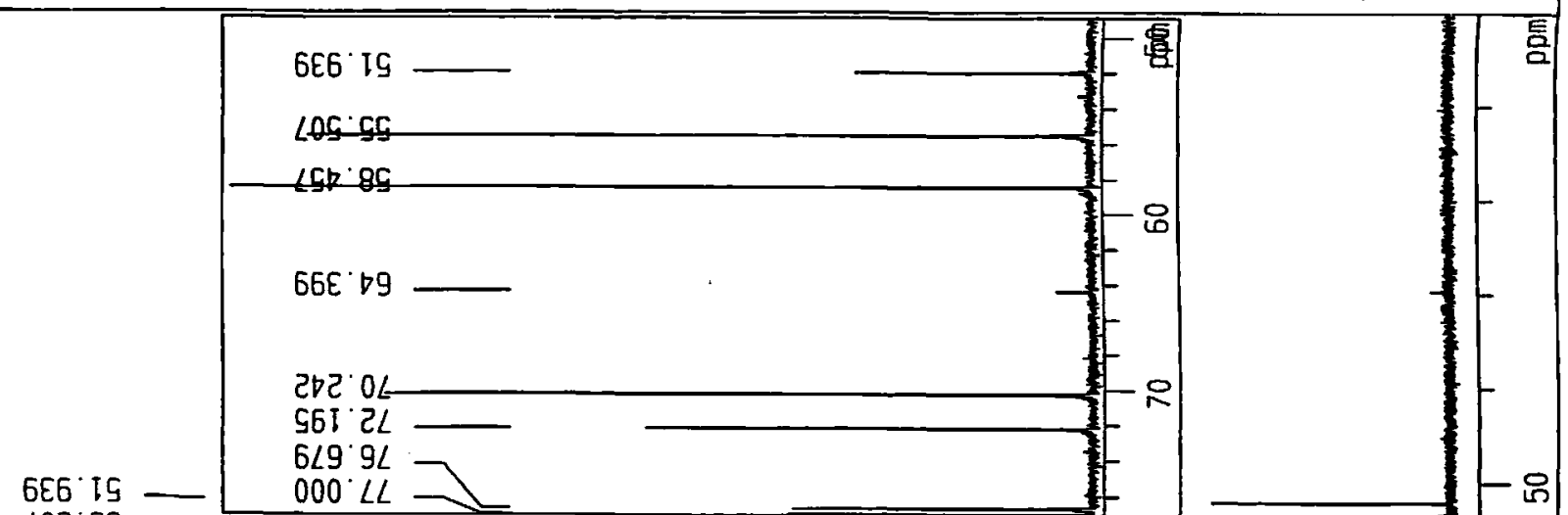

$\angle O G$ SG

$\angle S B \cdot B G=$

$66 E^{\circ} \cdot 79$

टमट OL

S6I. 22

$6 \angle 9.9 L$

$000^{\circ} \angle L$

IटE' $L L$

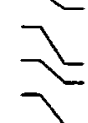




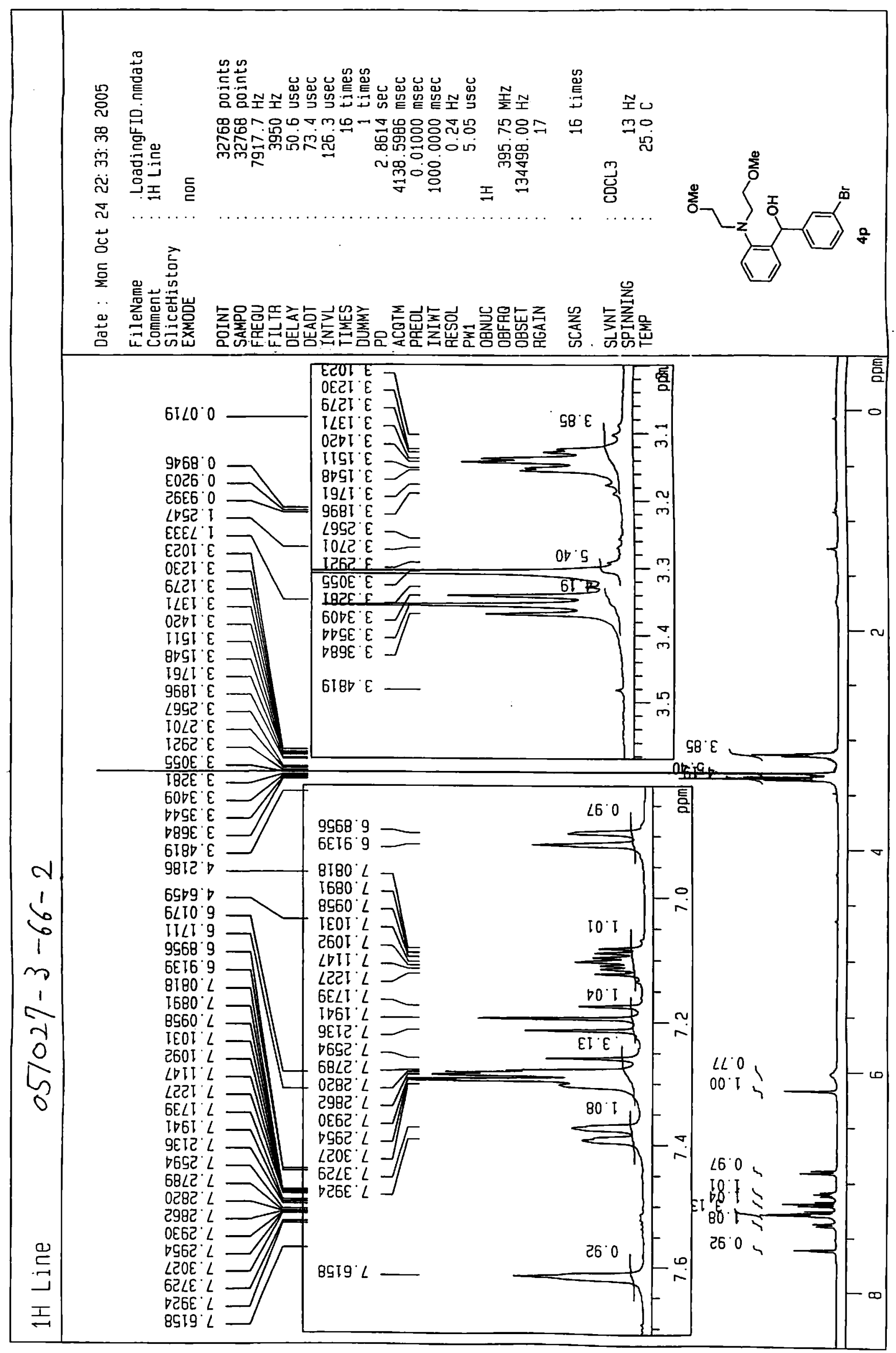




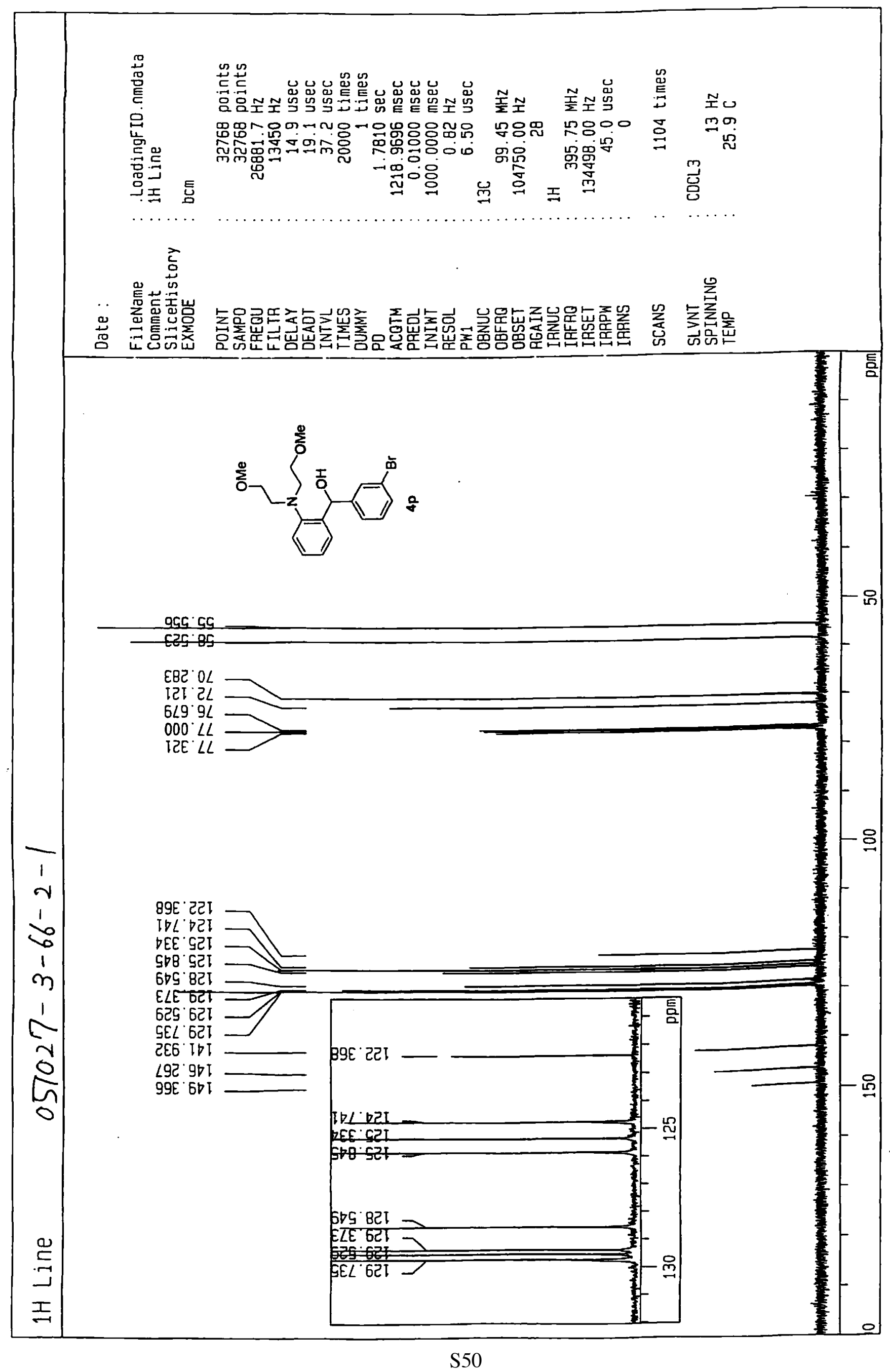




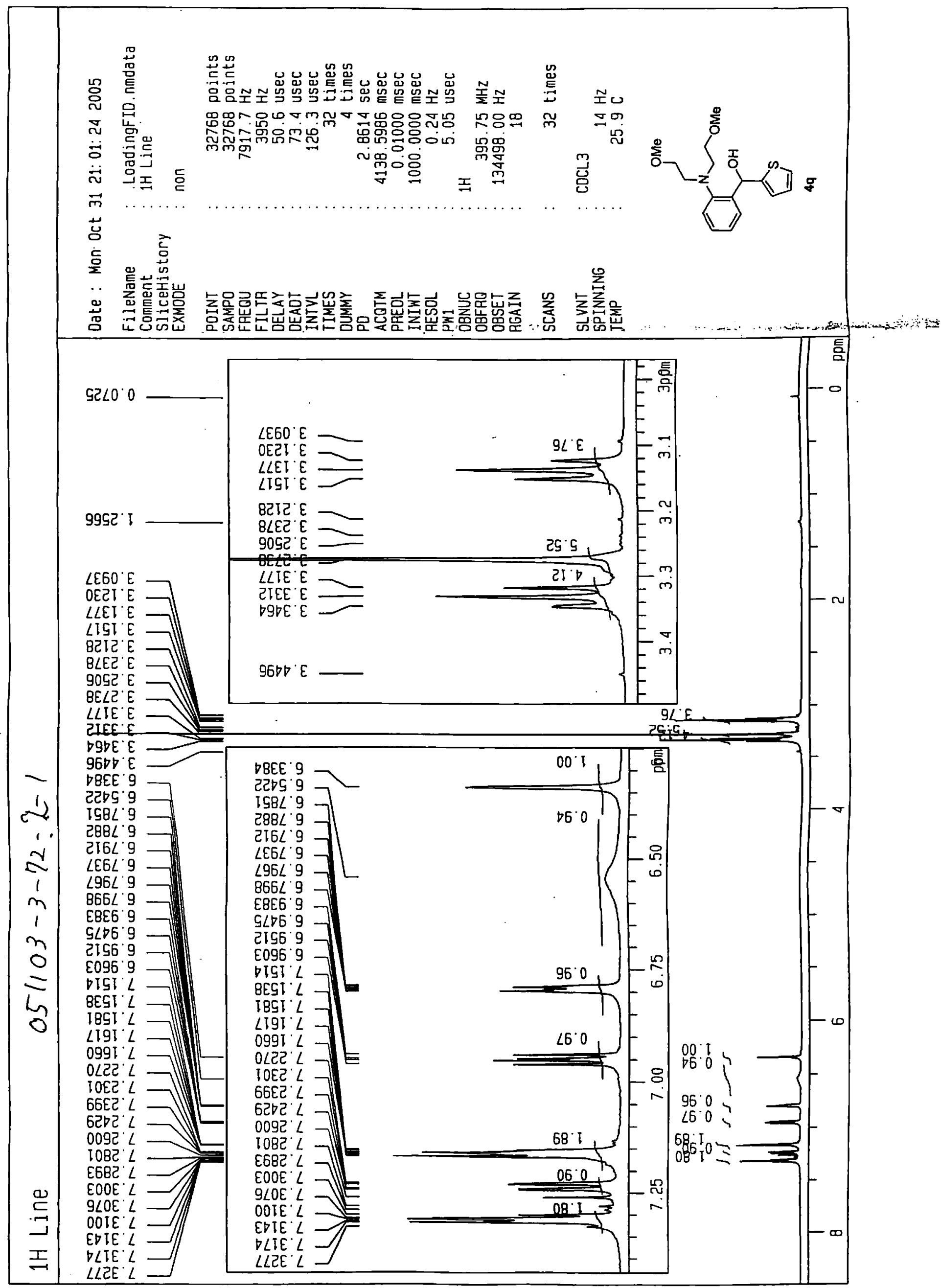




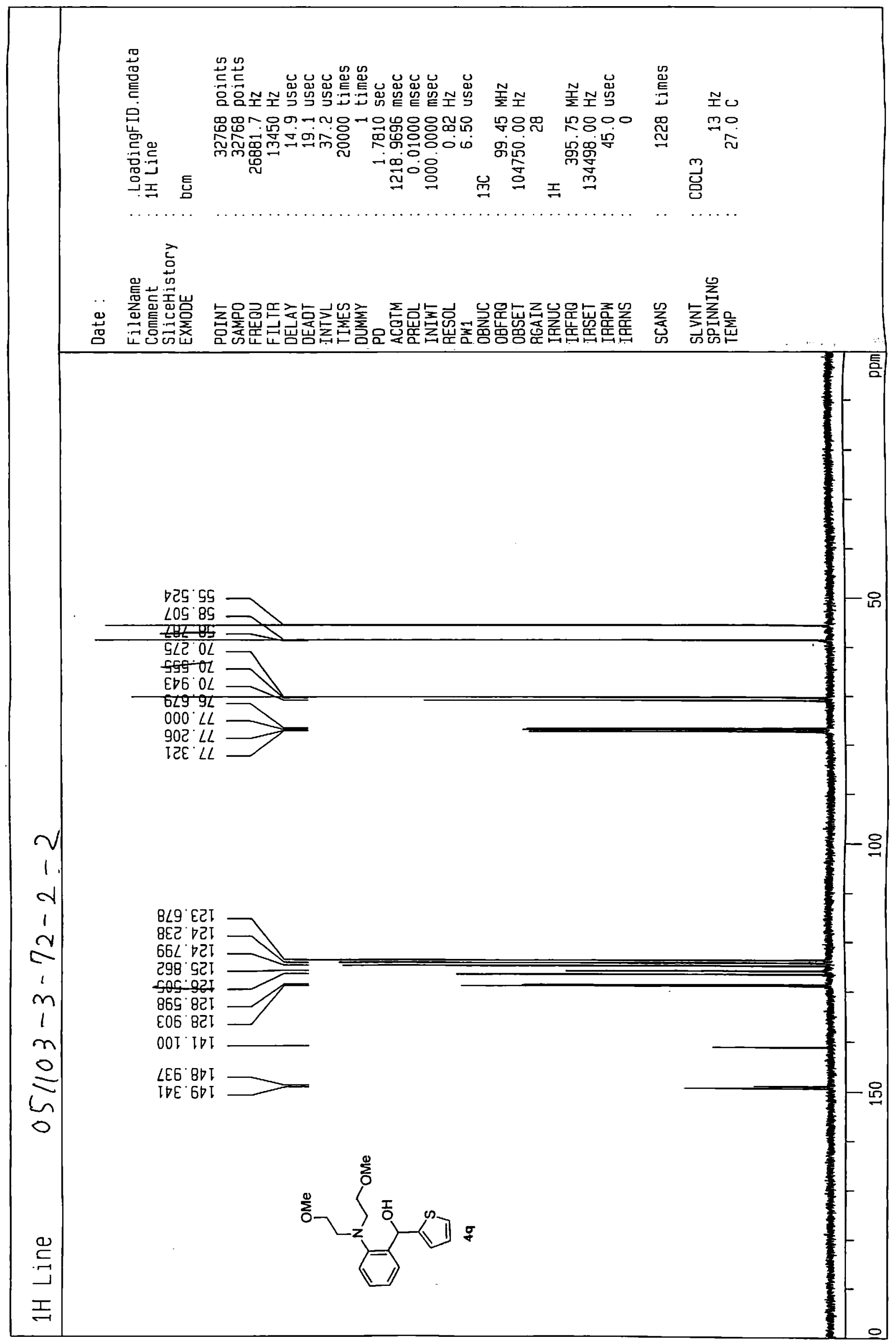




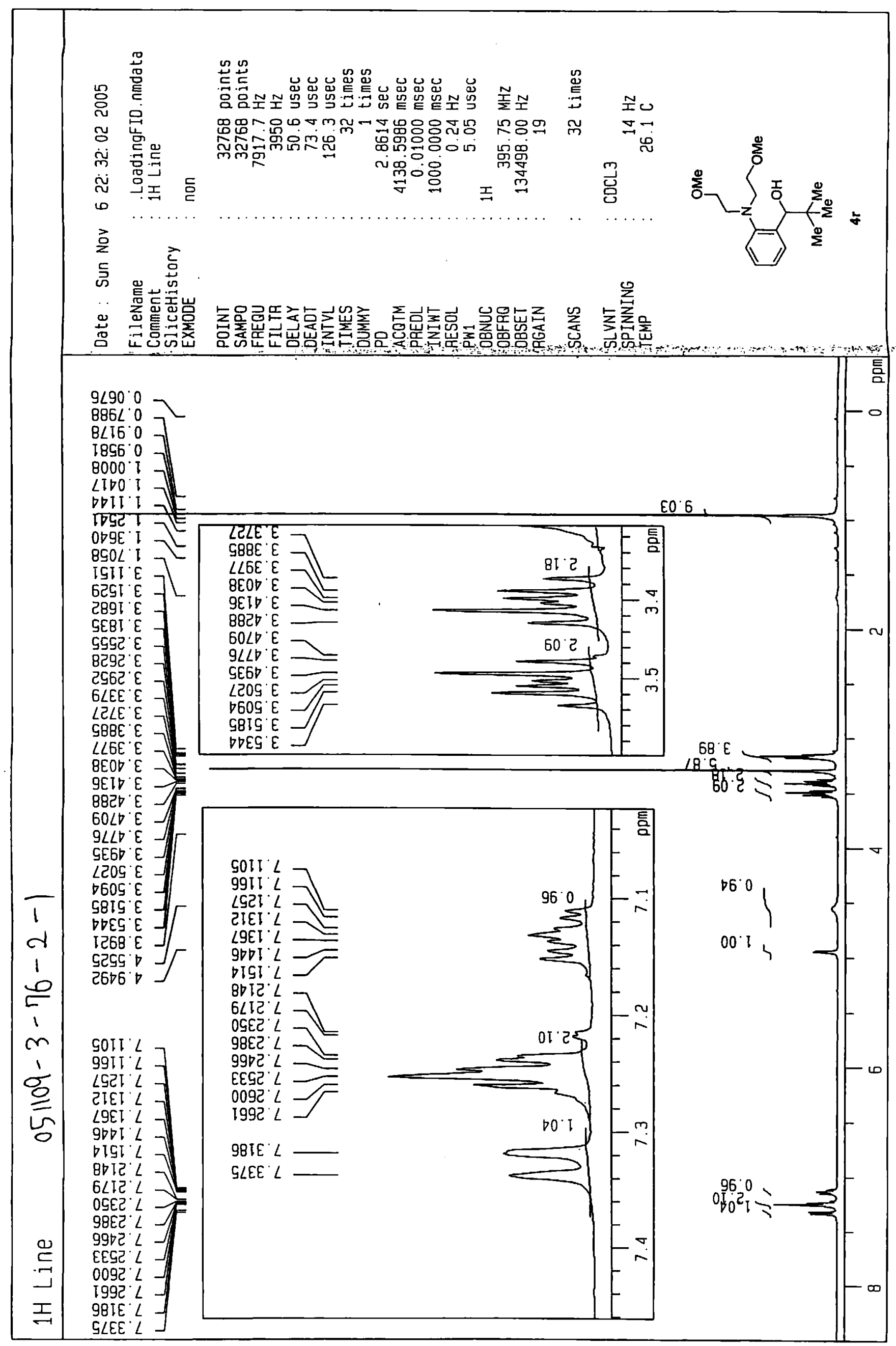




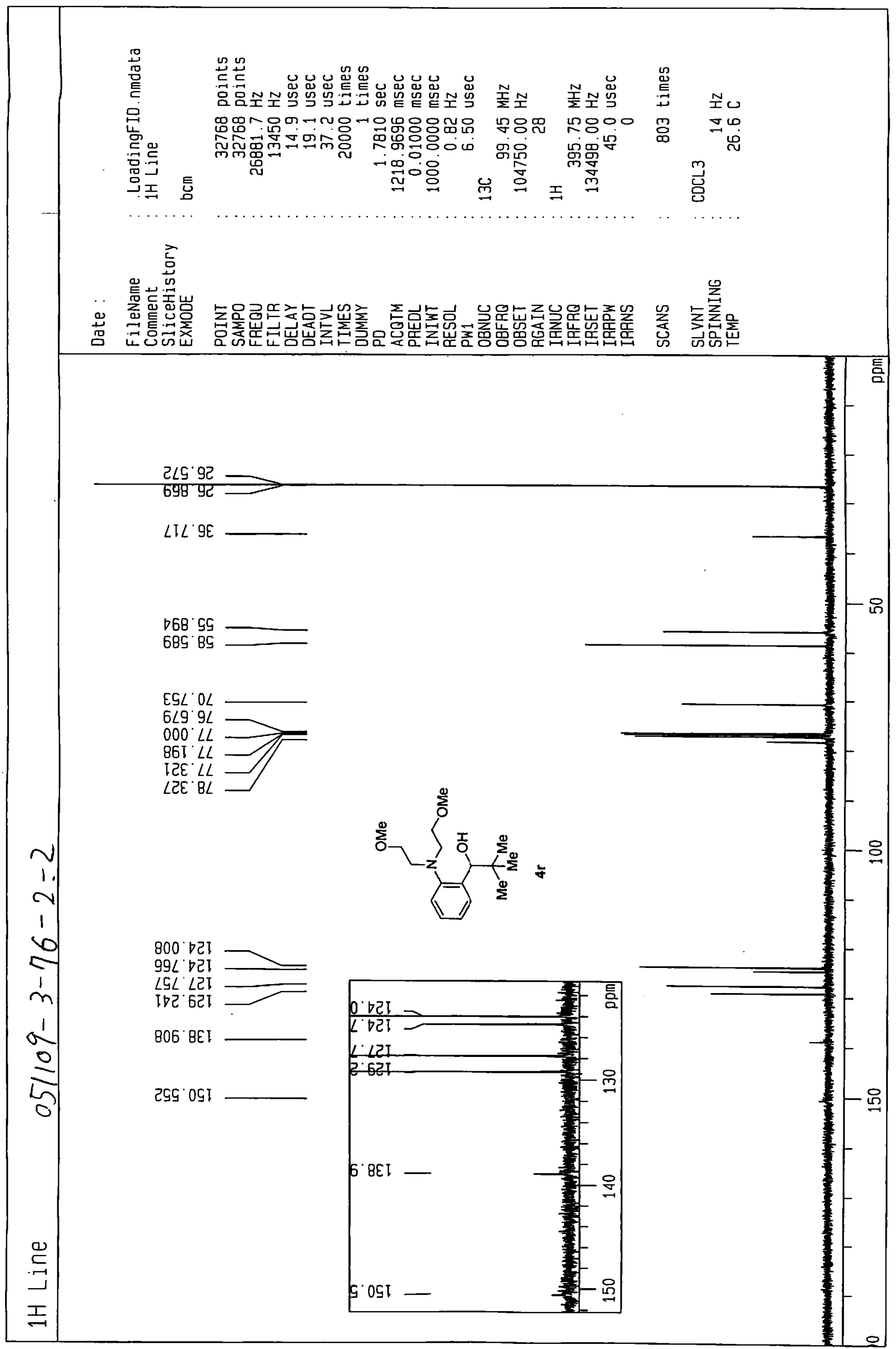




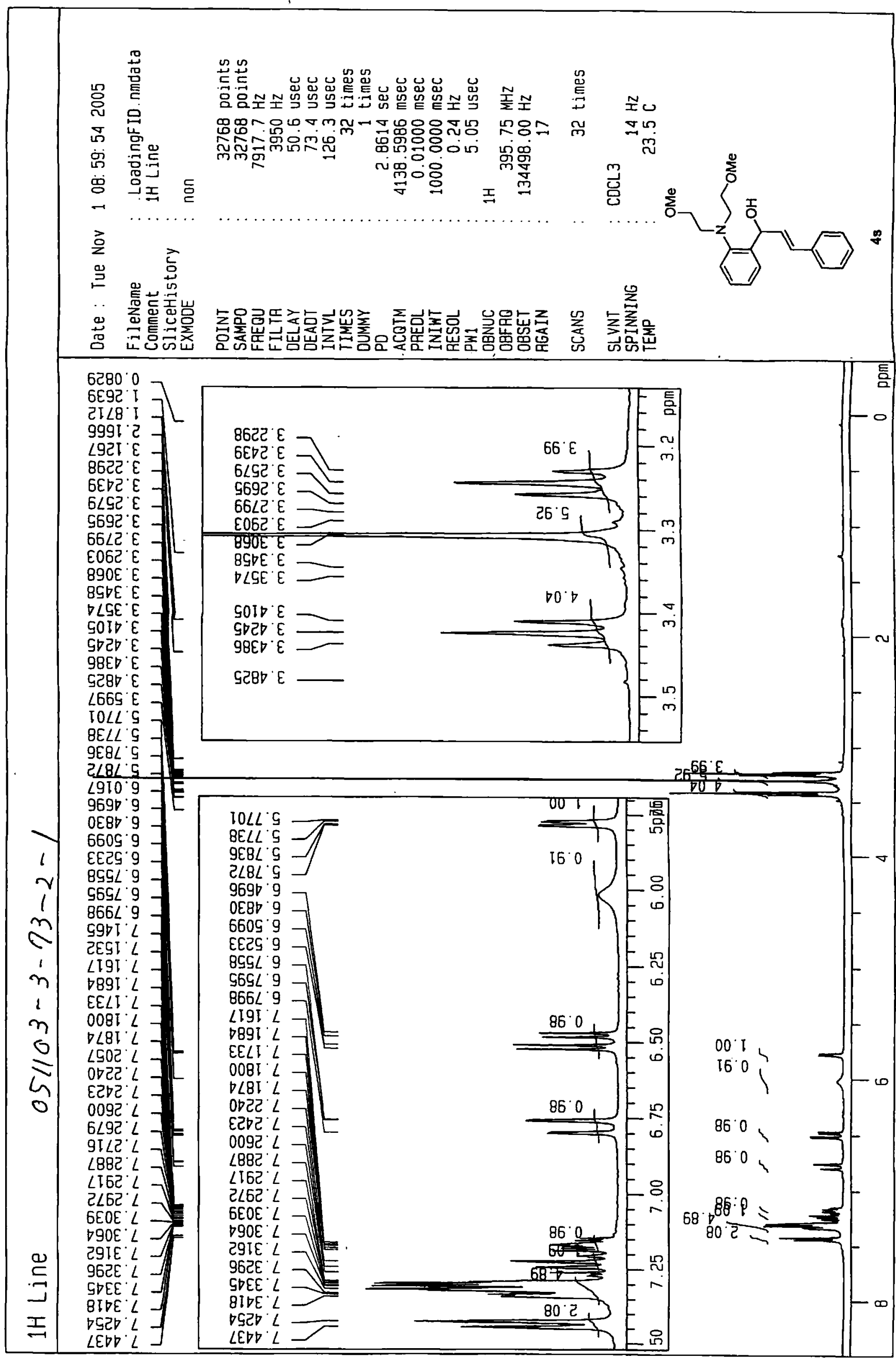




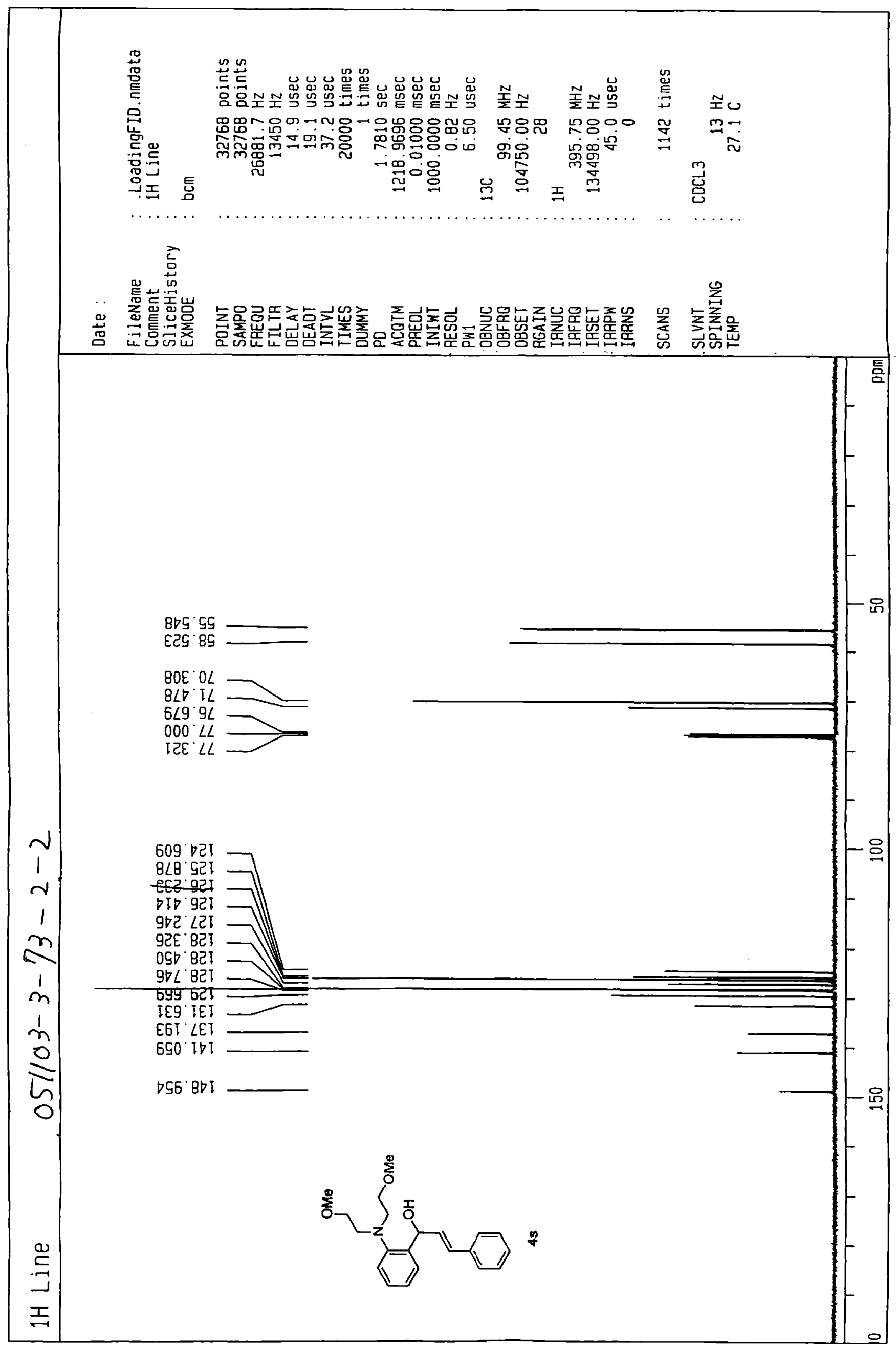




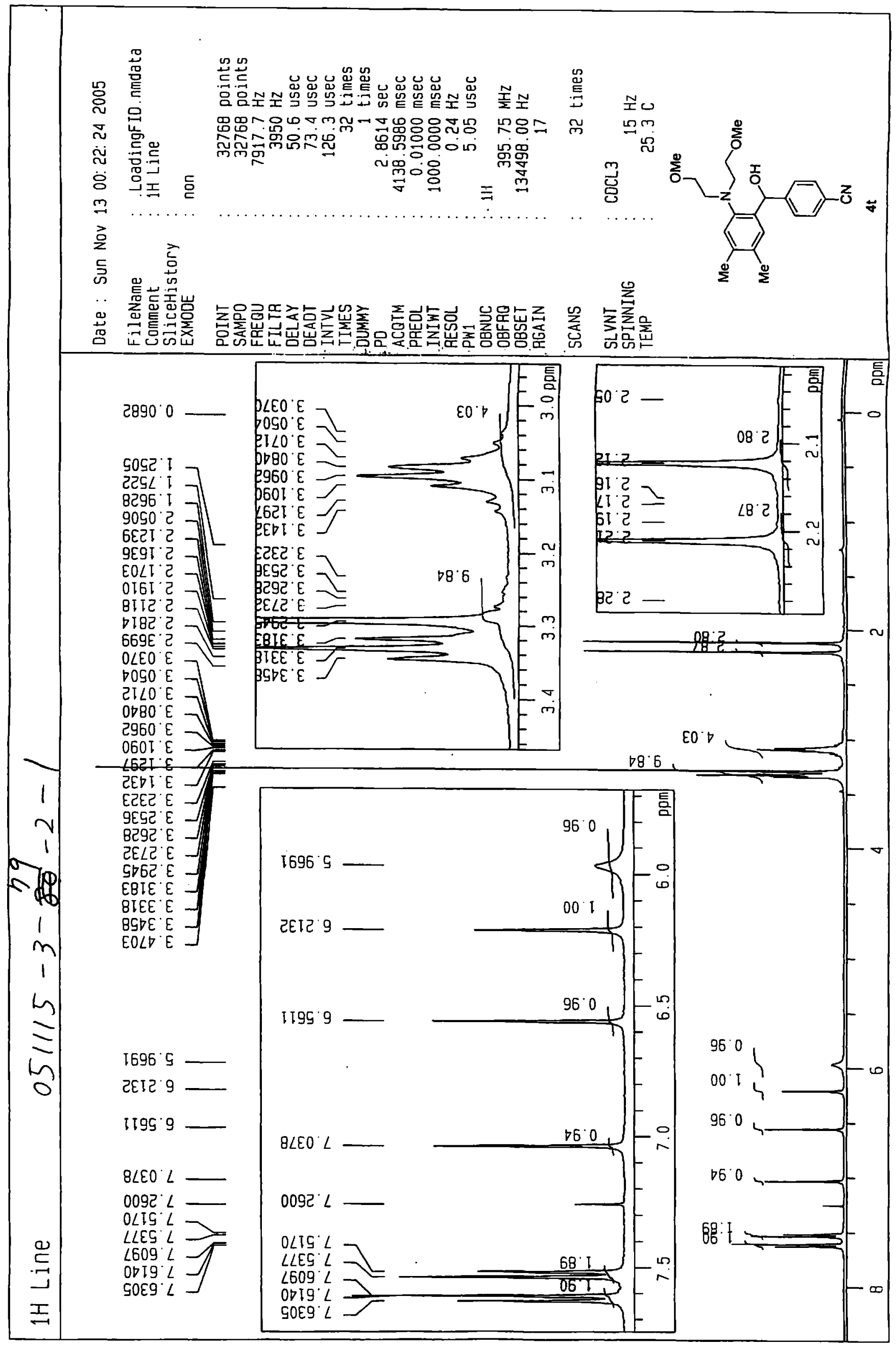




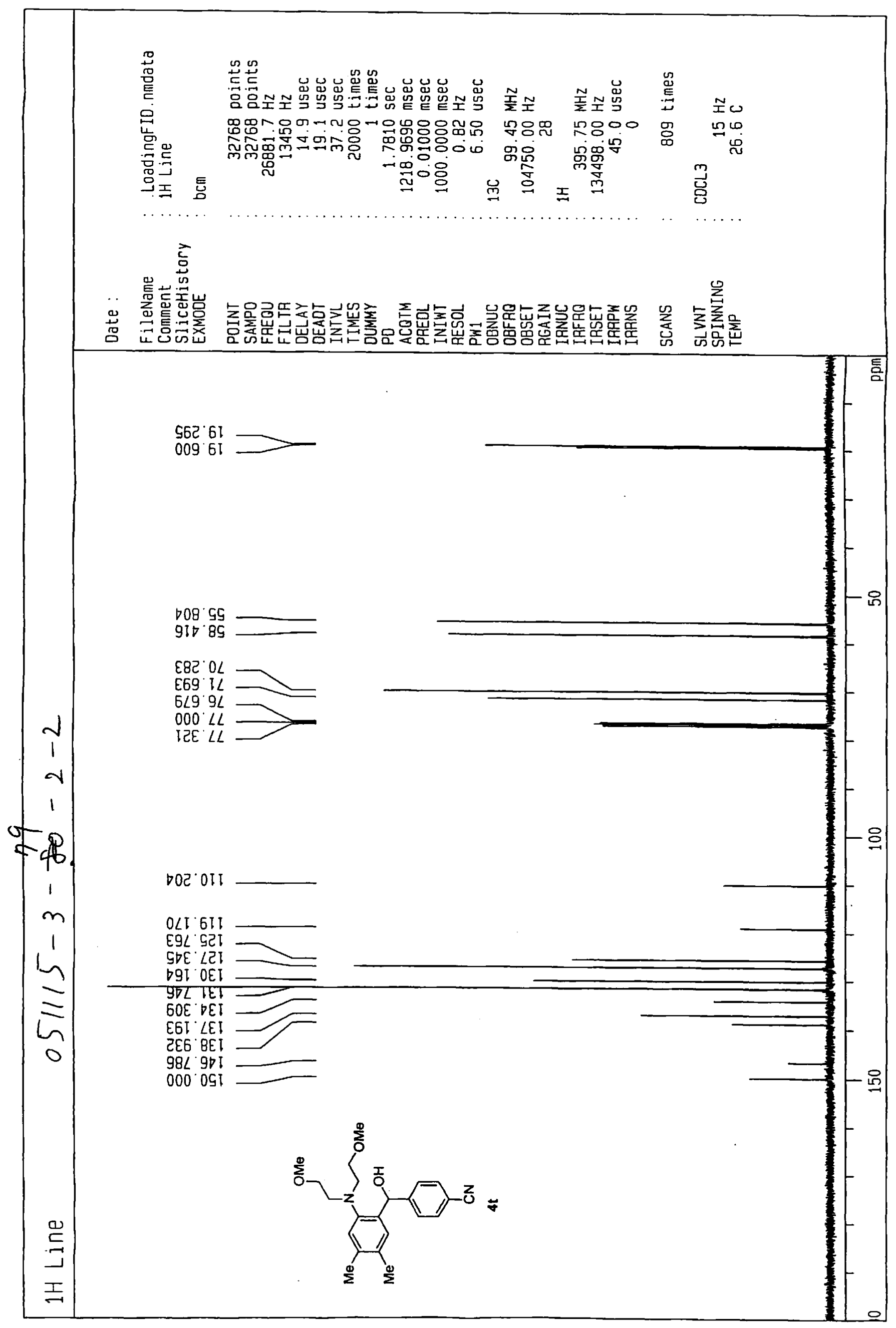




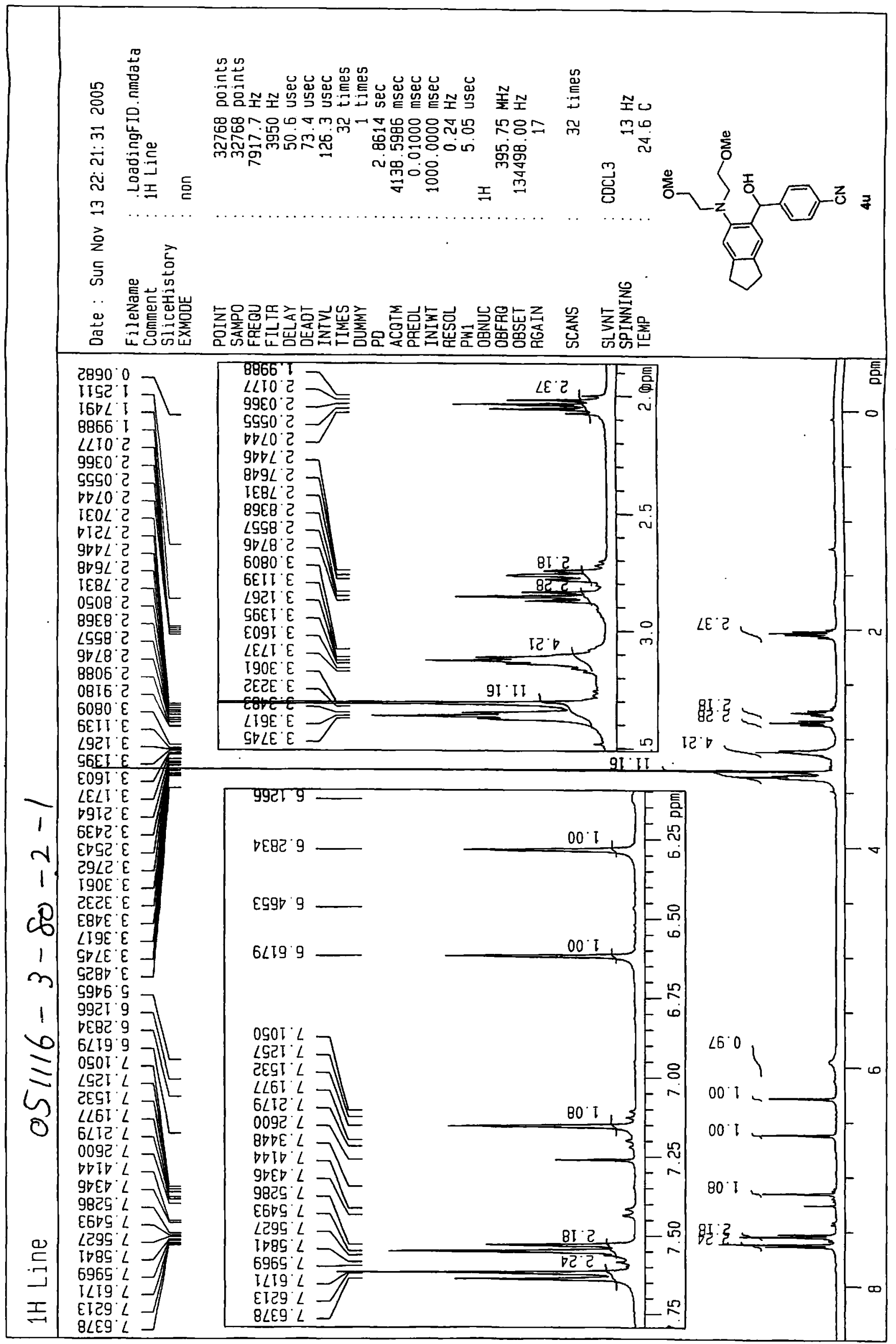




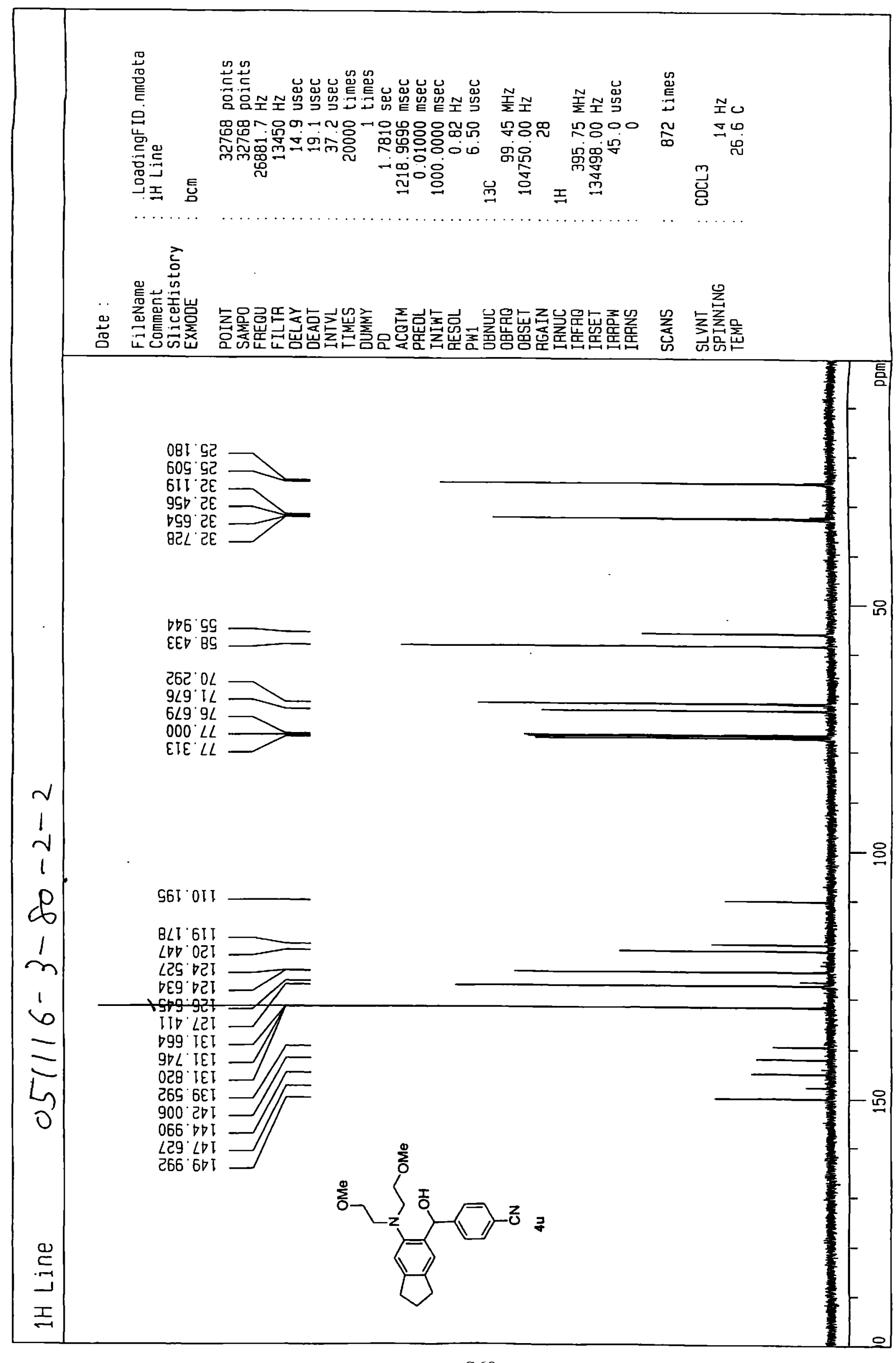




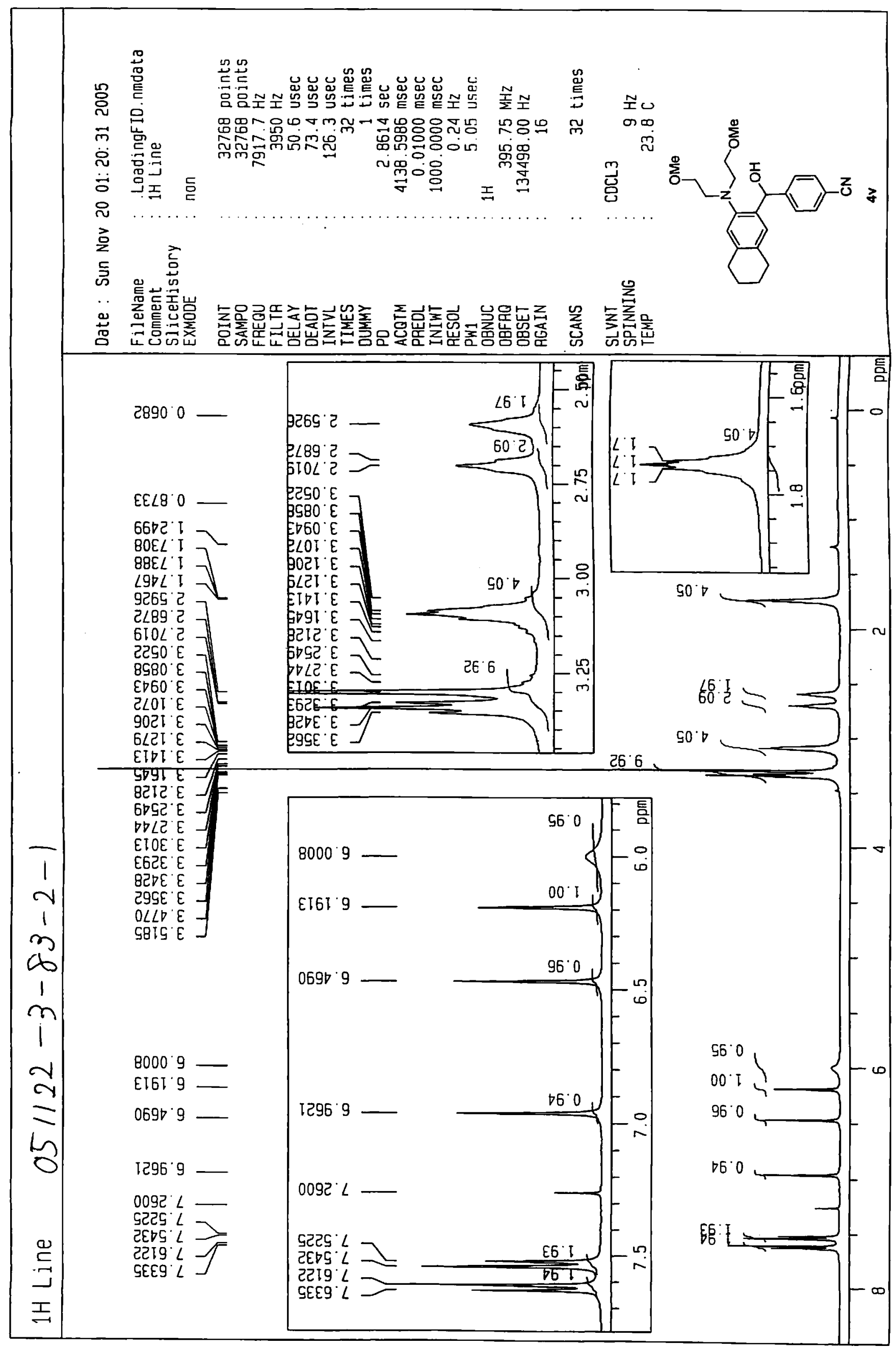




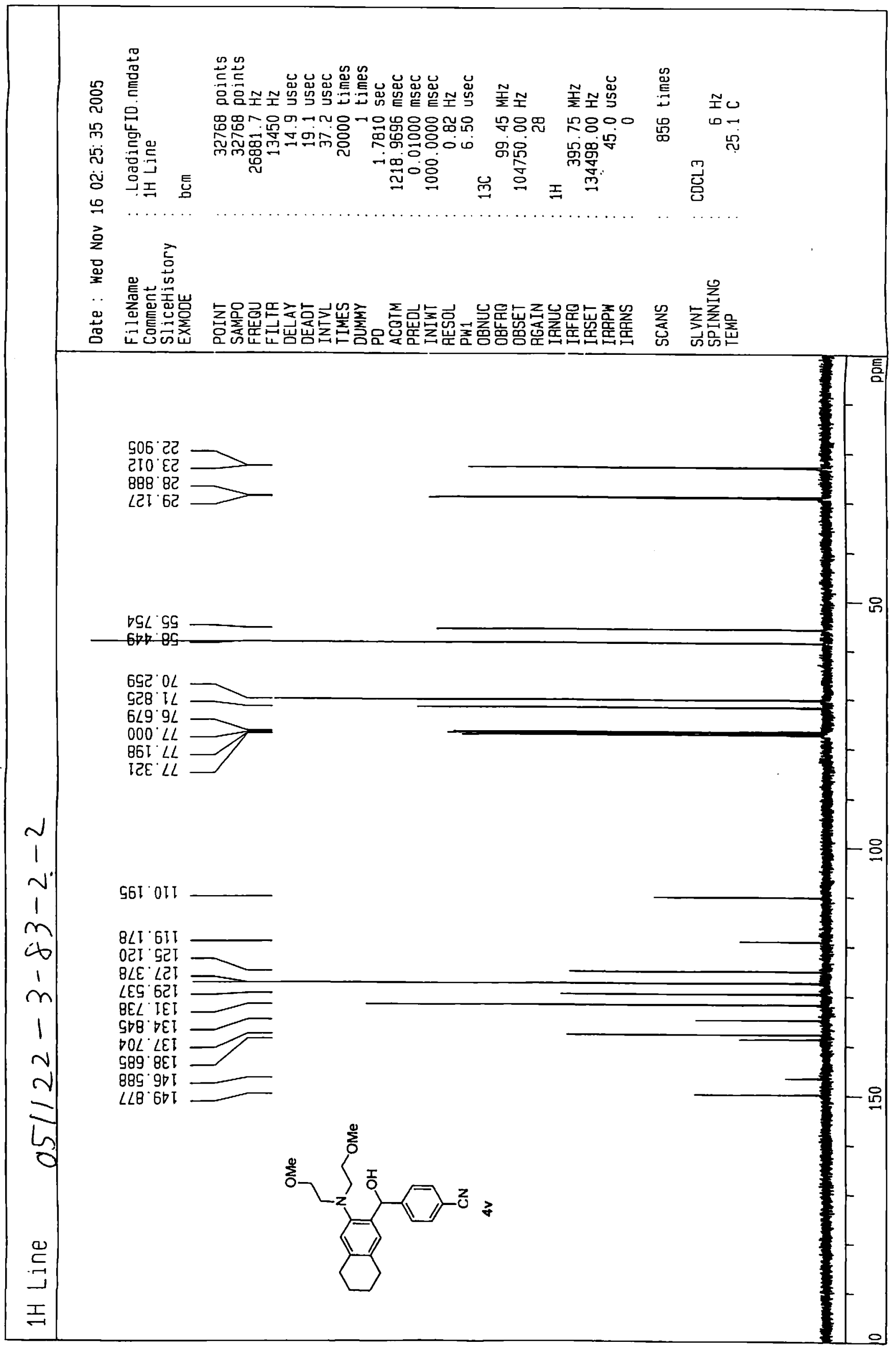




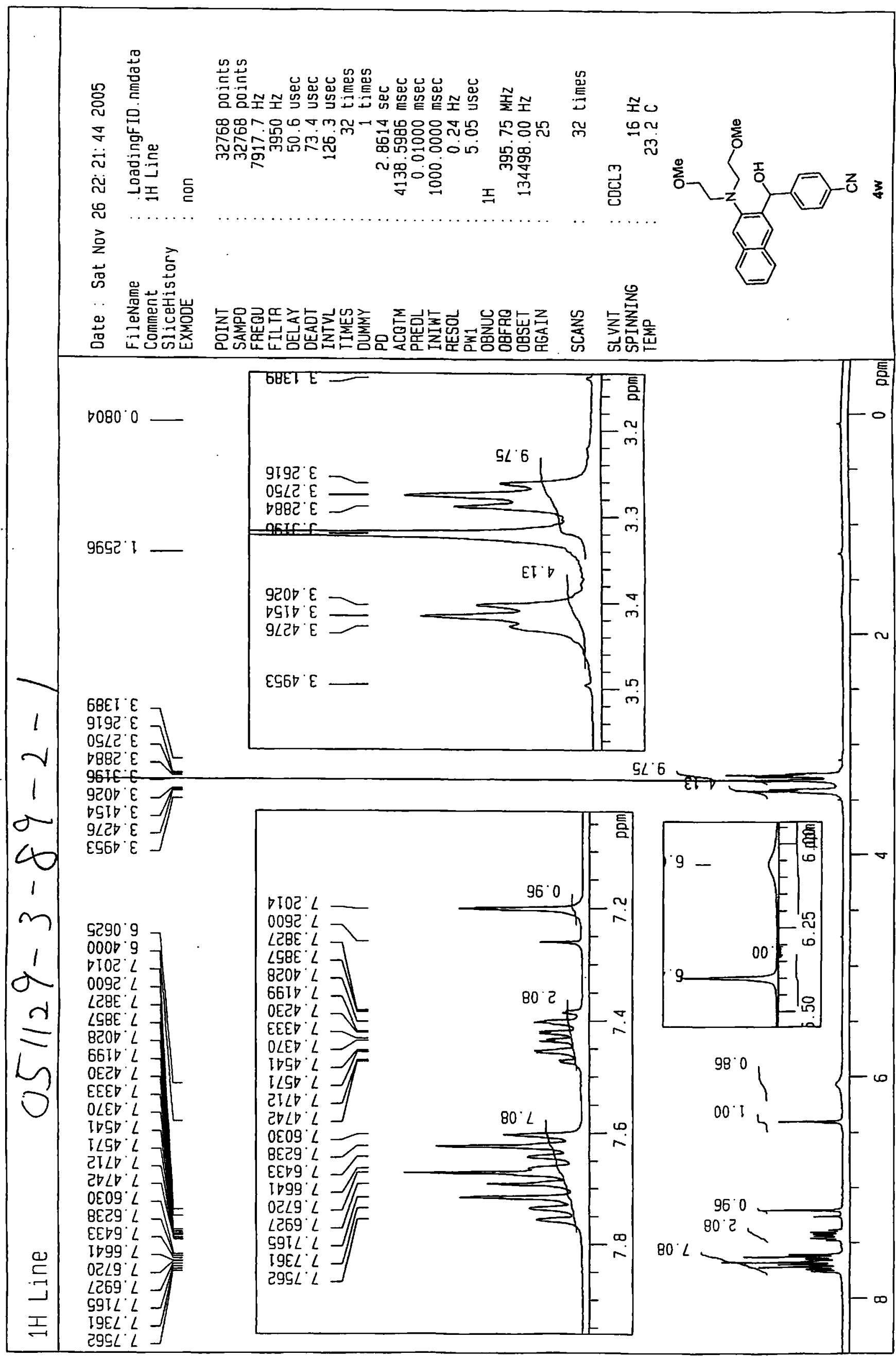




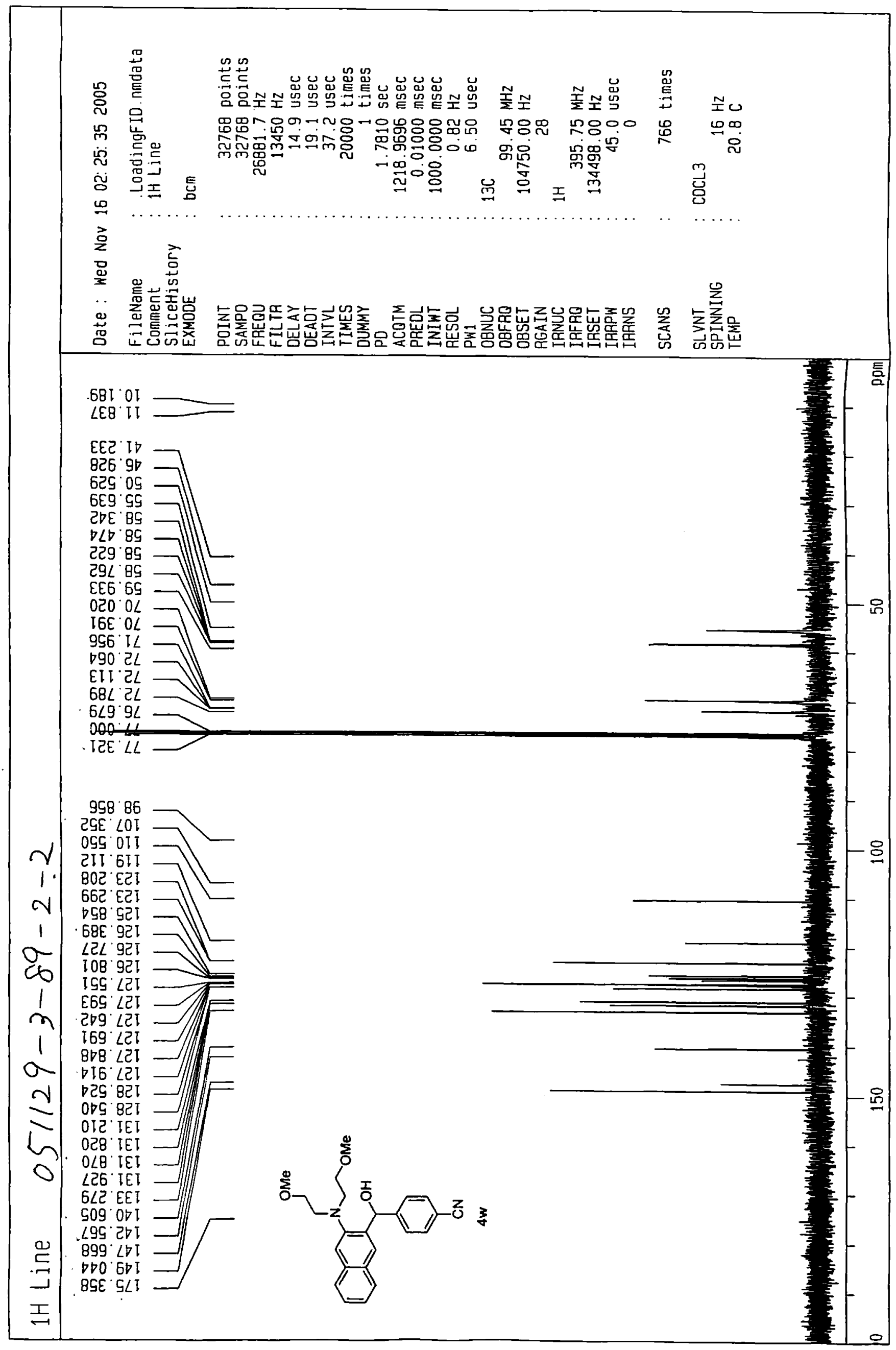




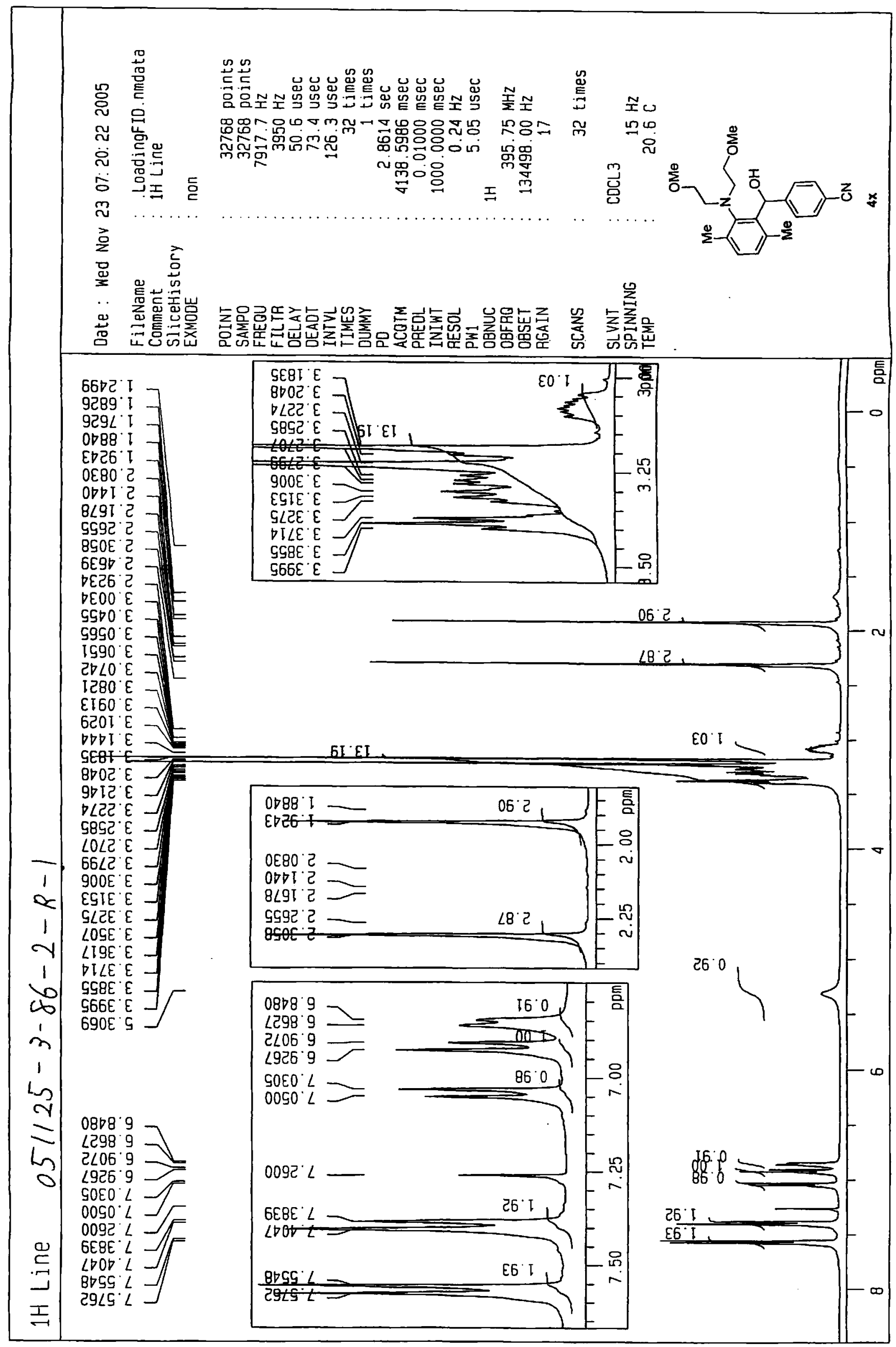




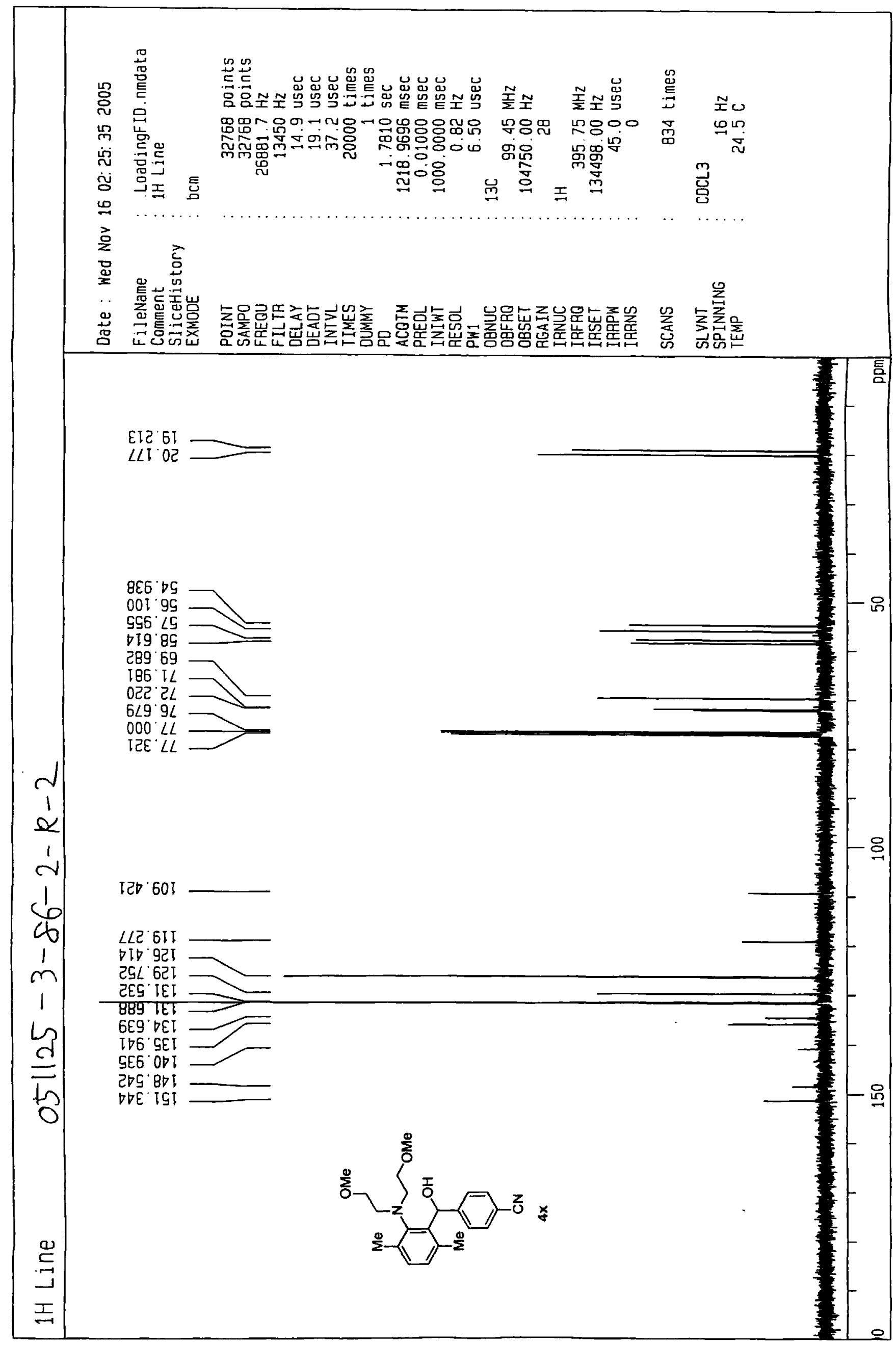




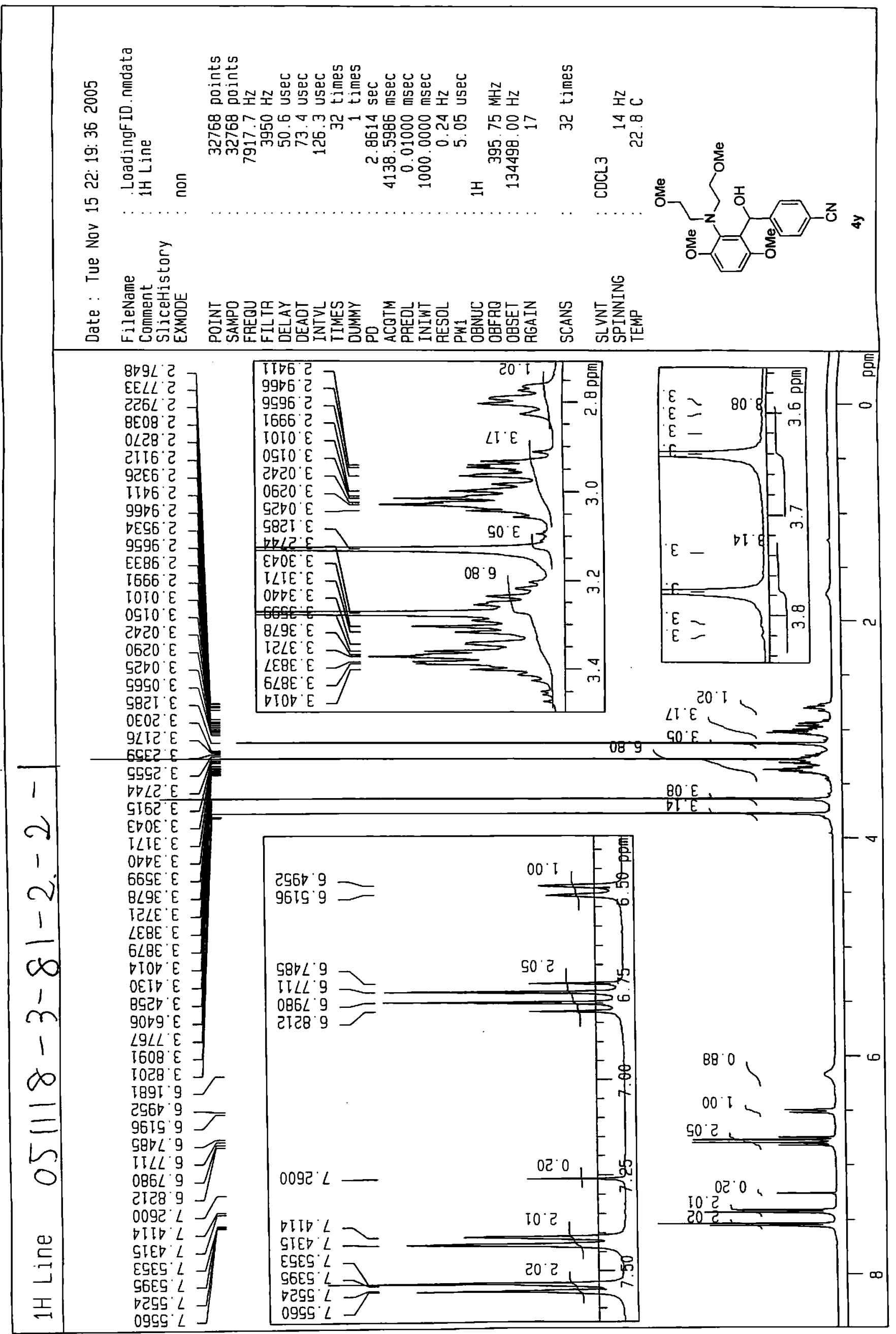




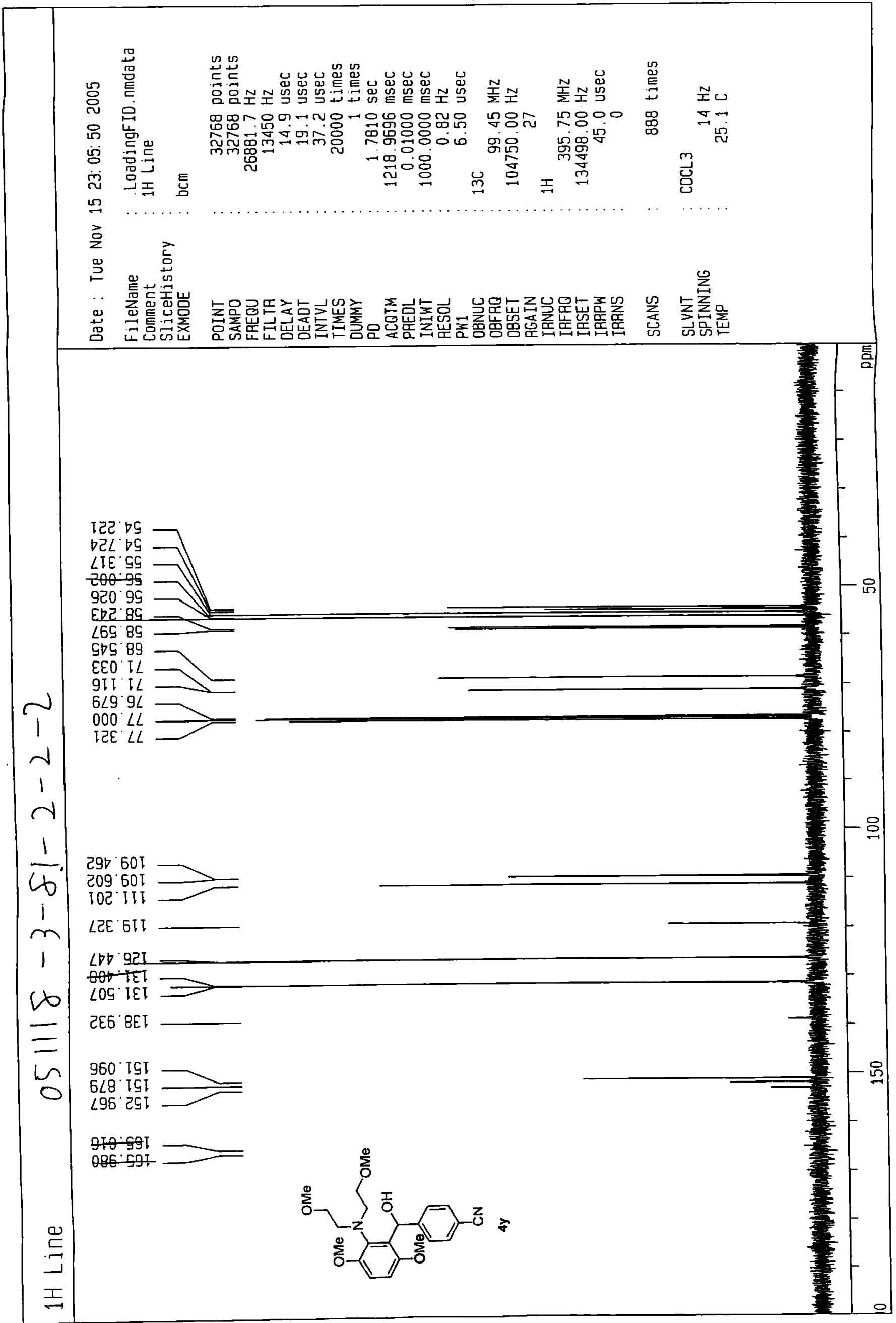




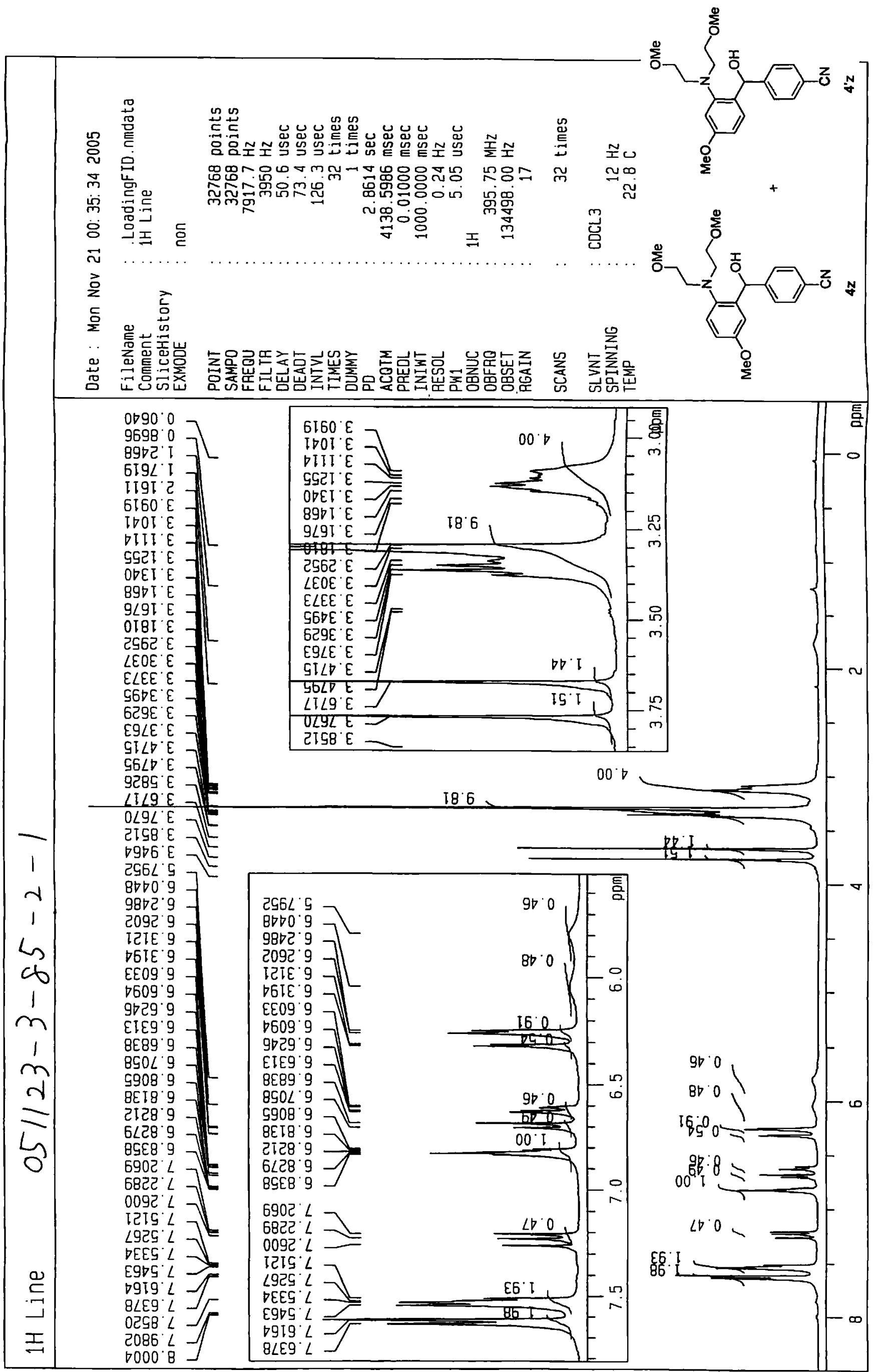




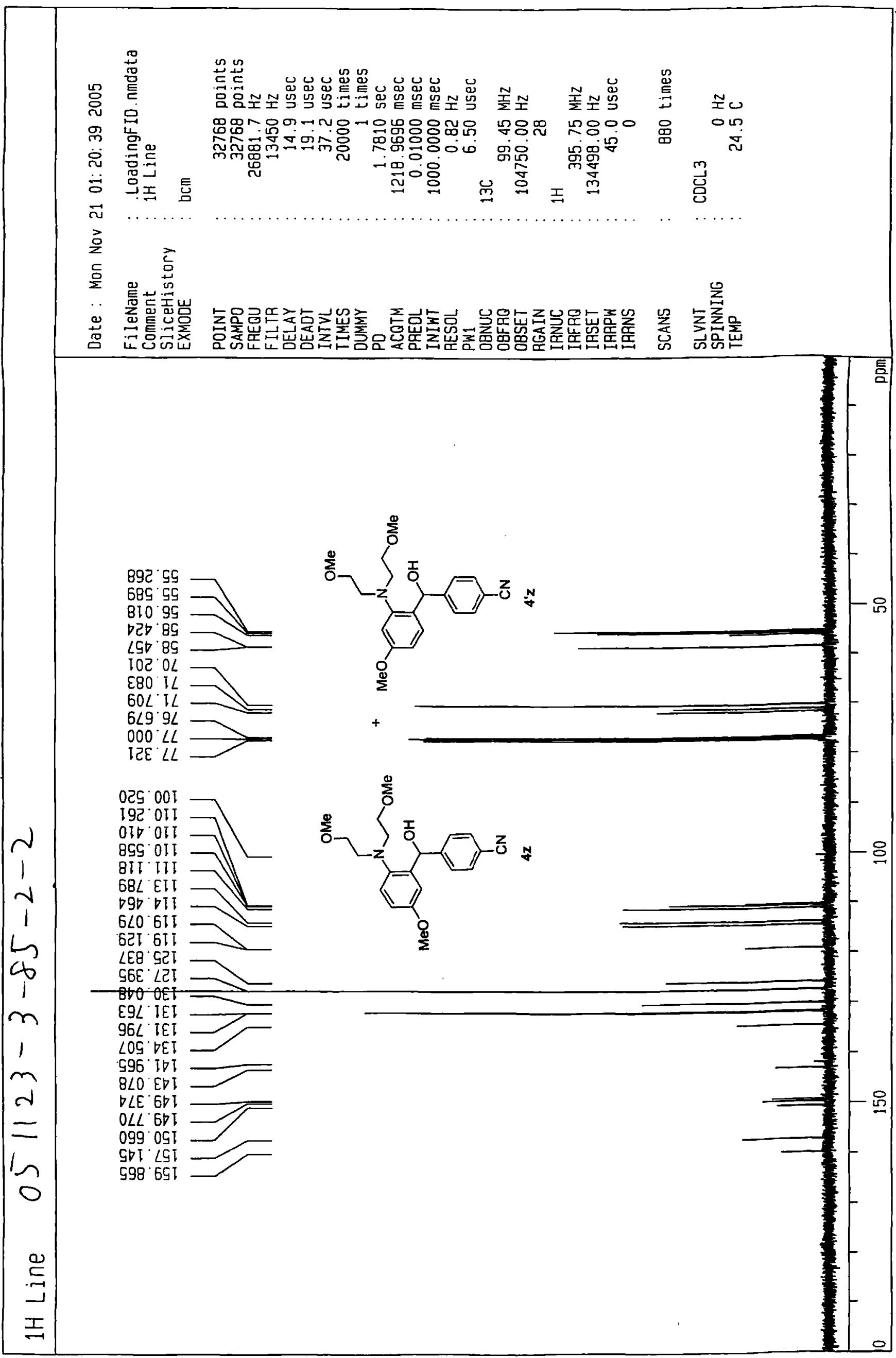




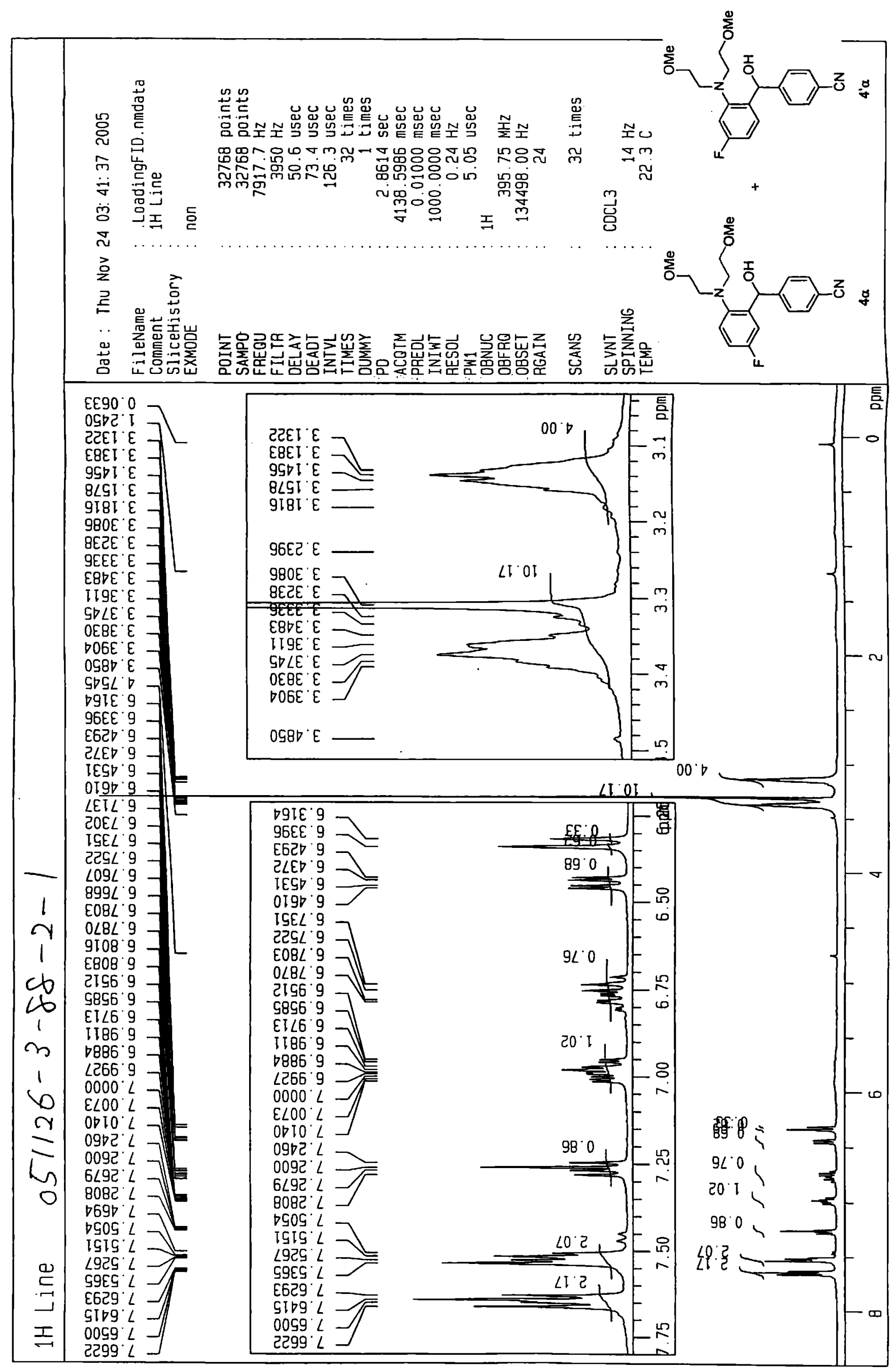




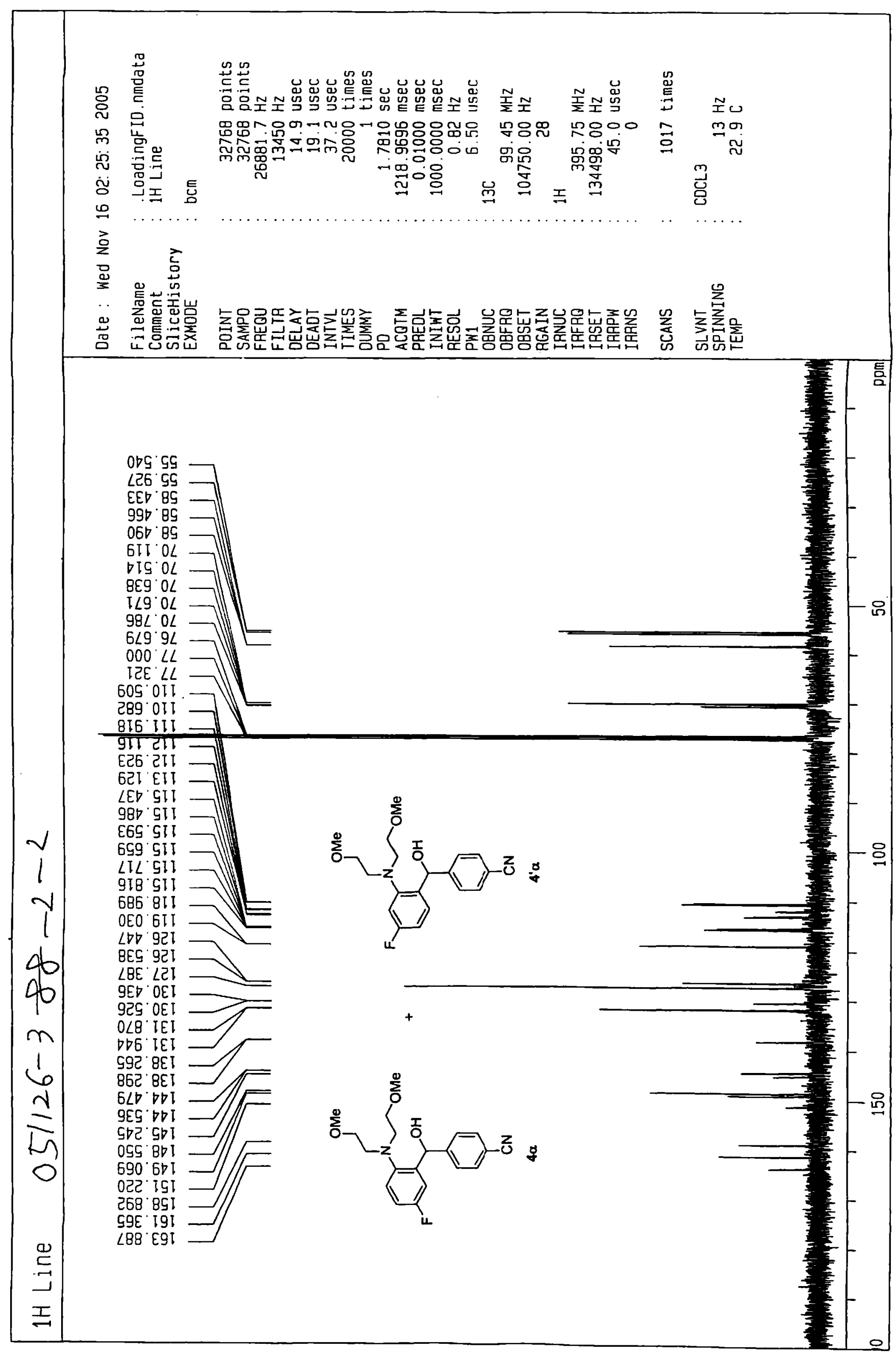




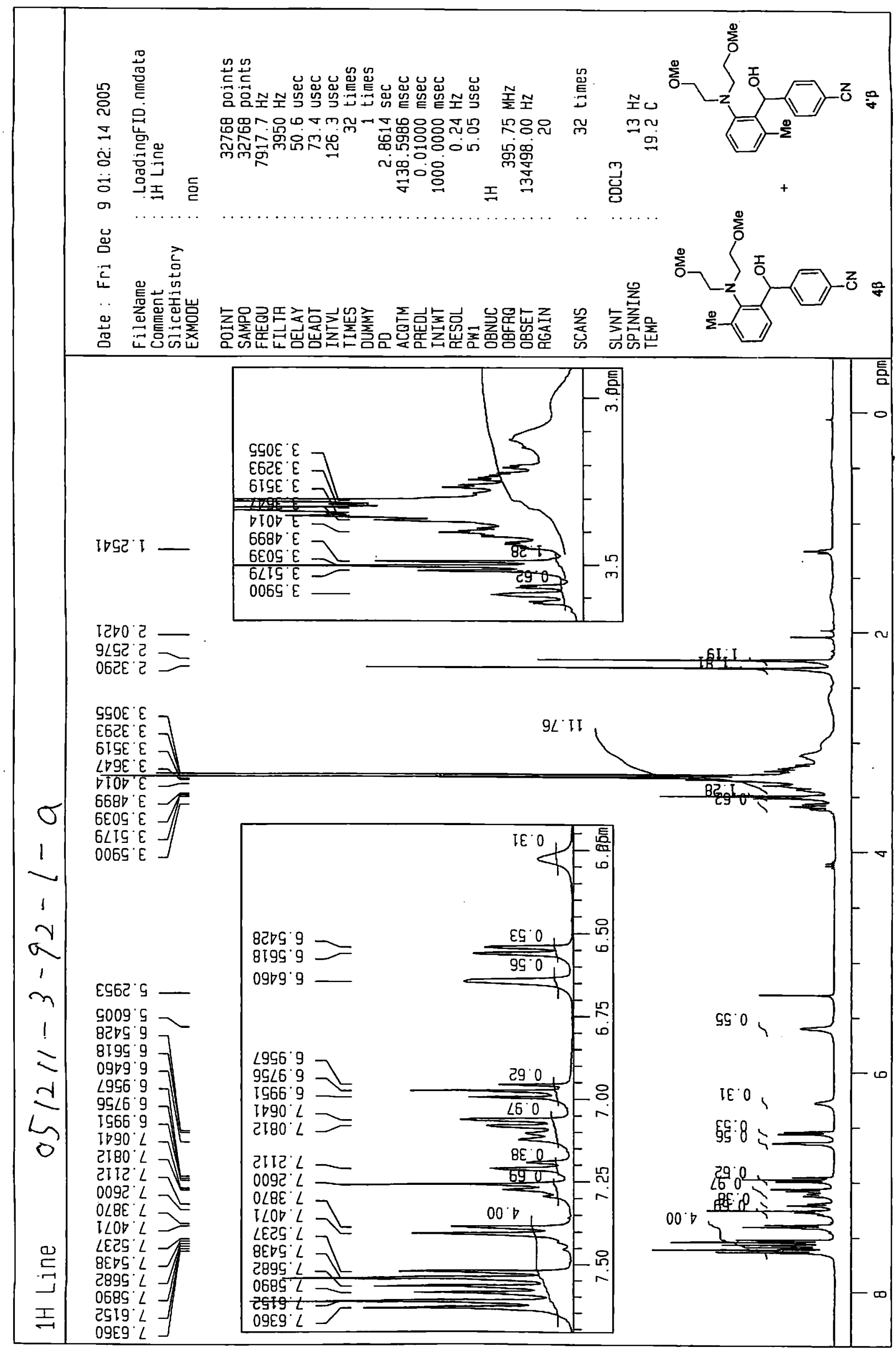




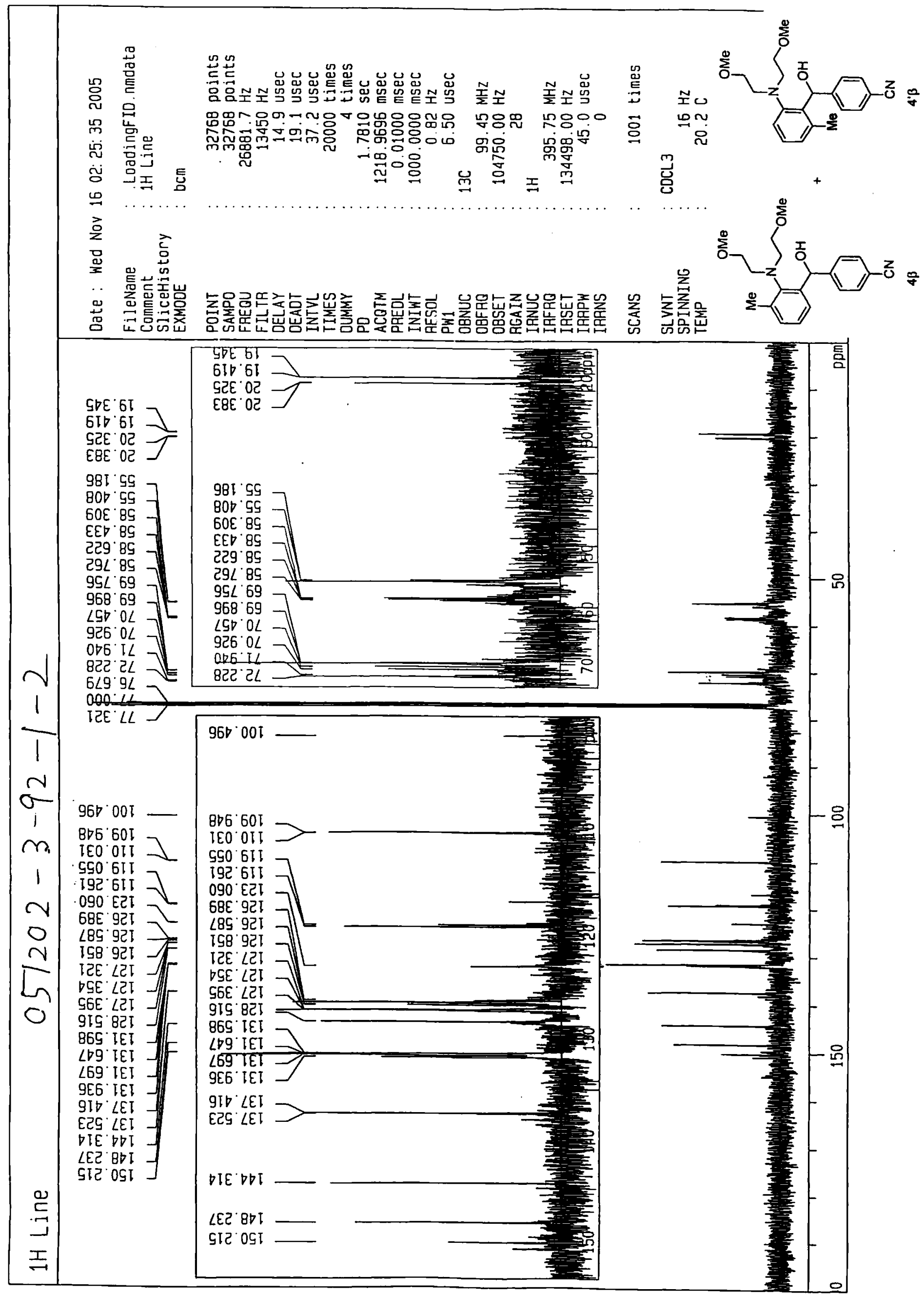




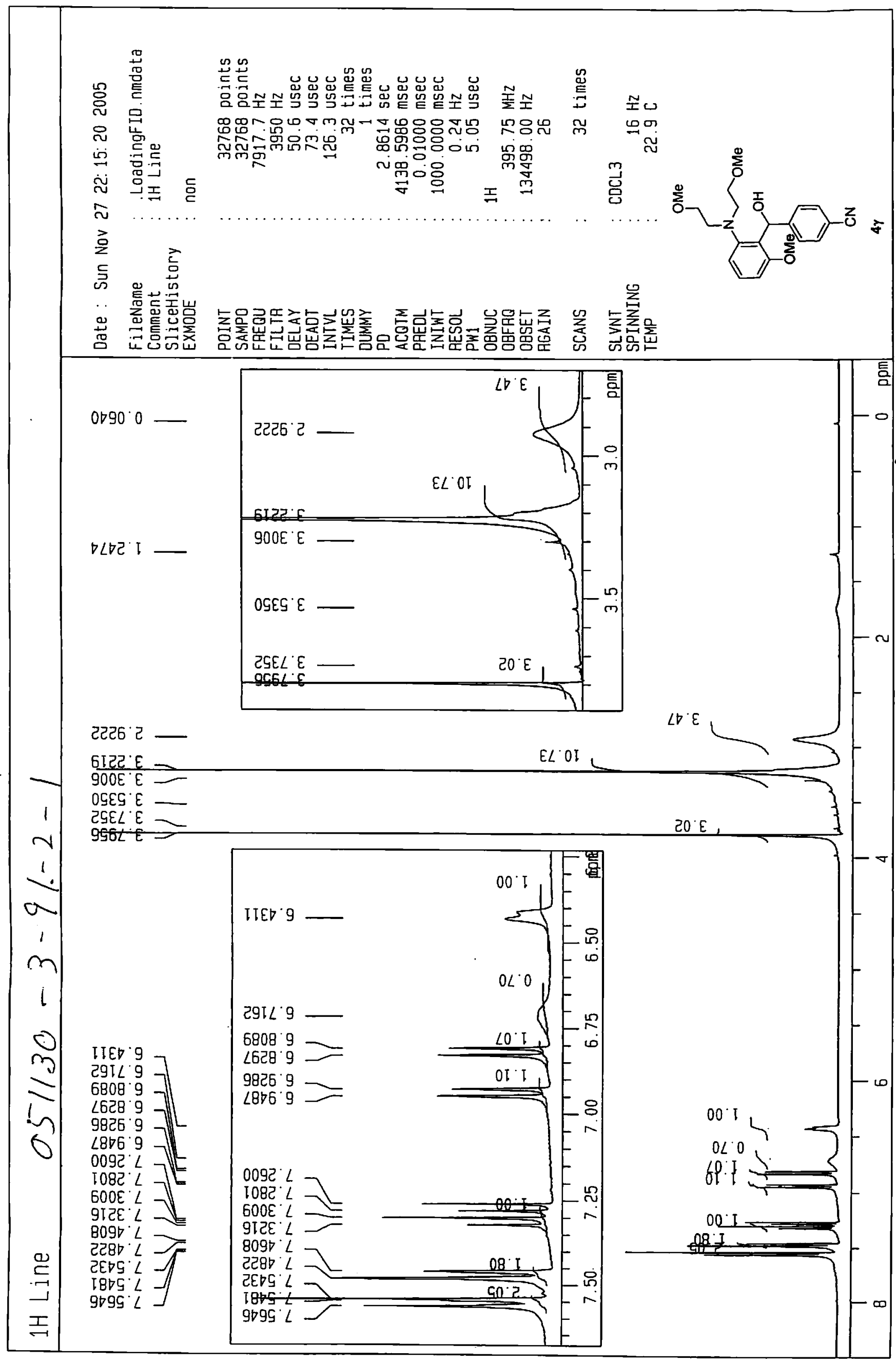




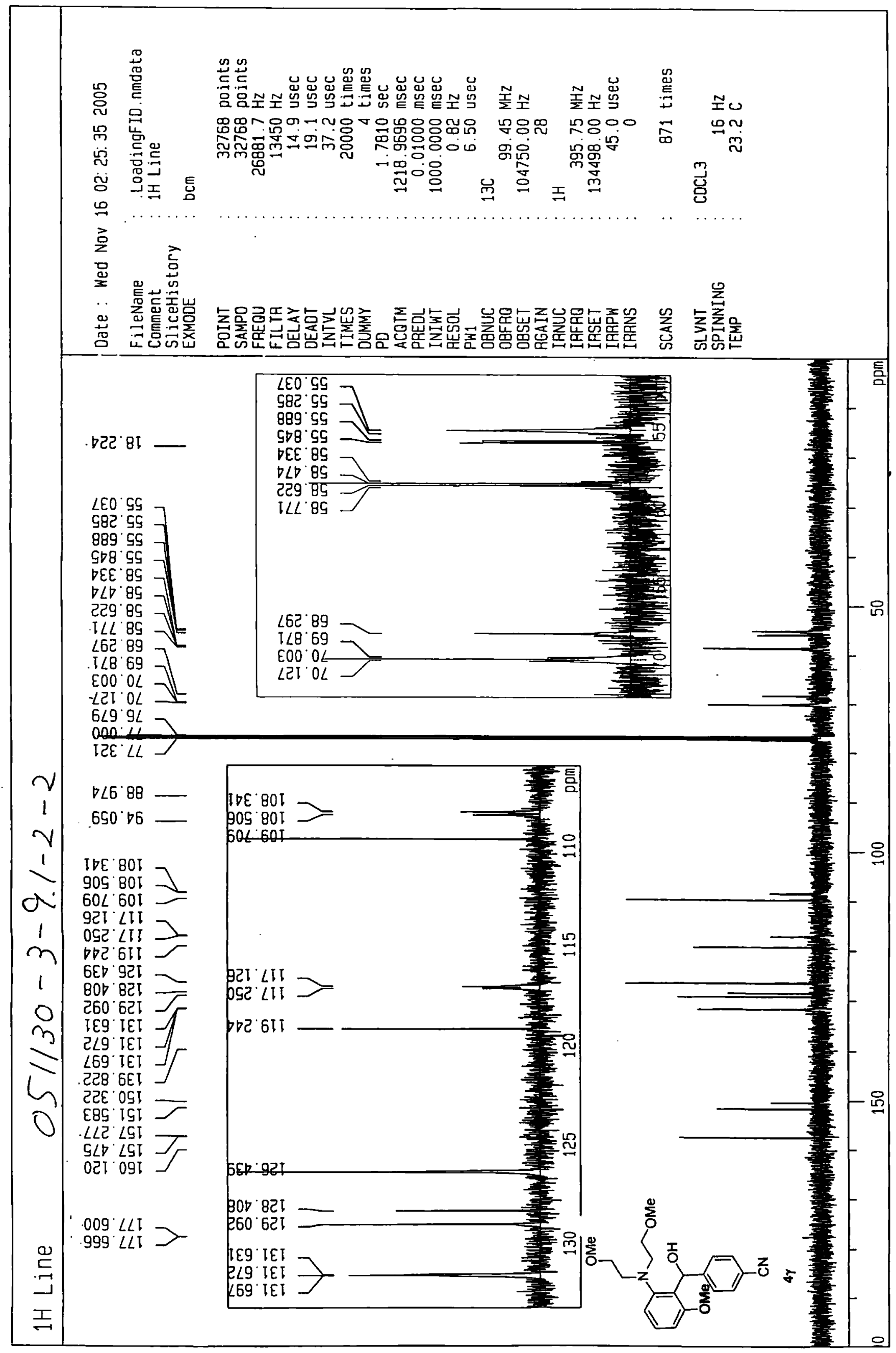




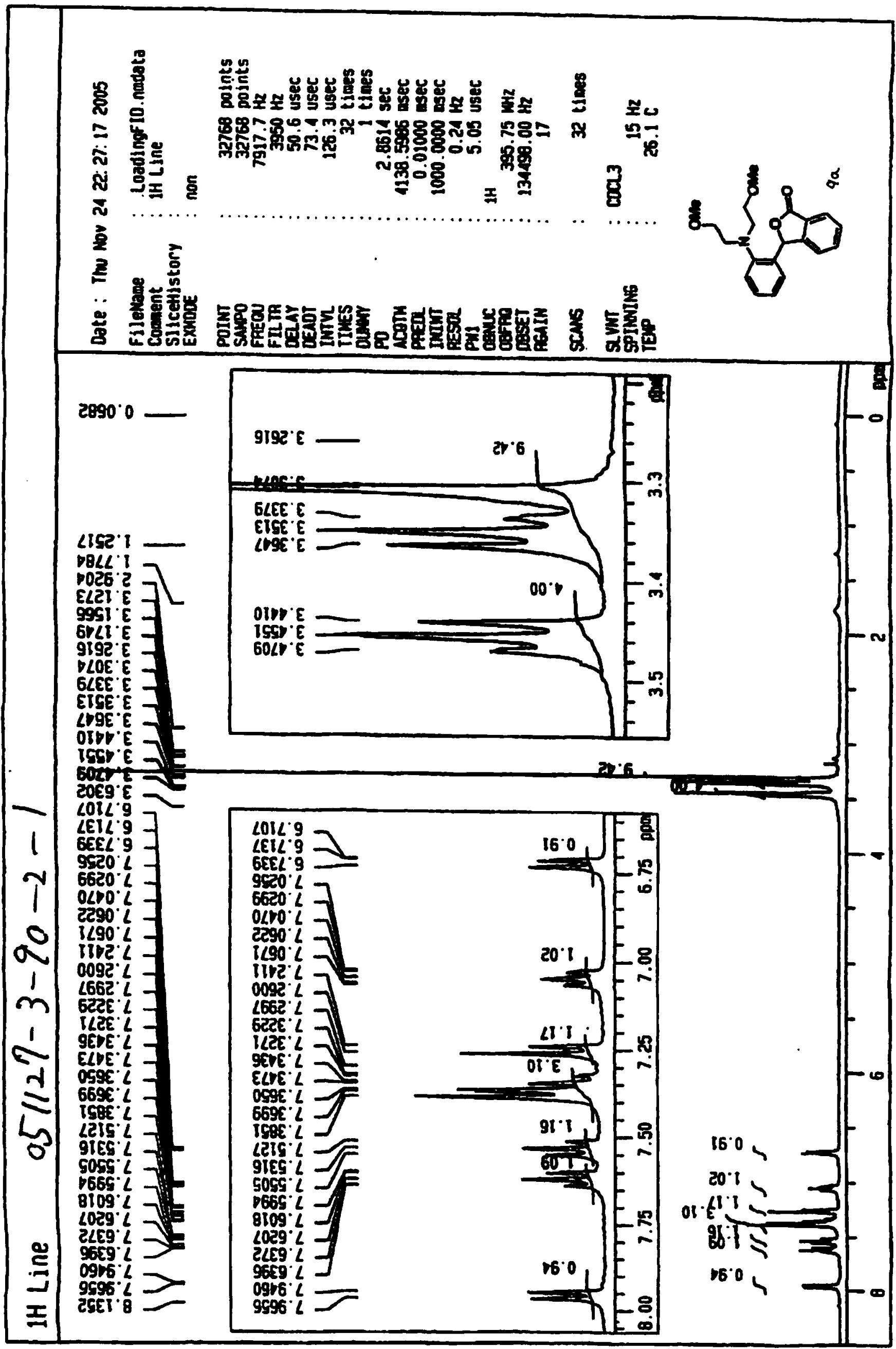




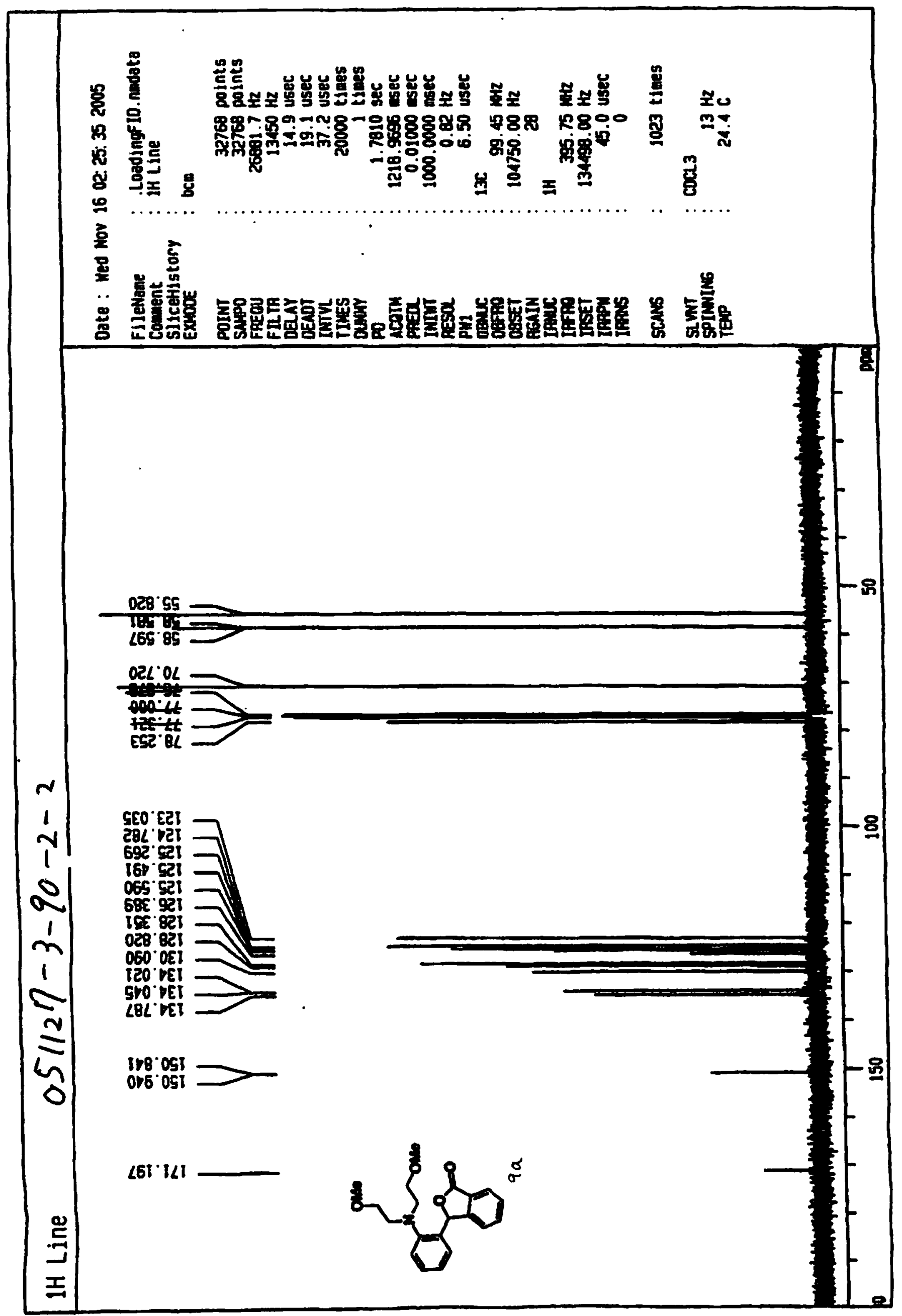




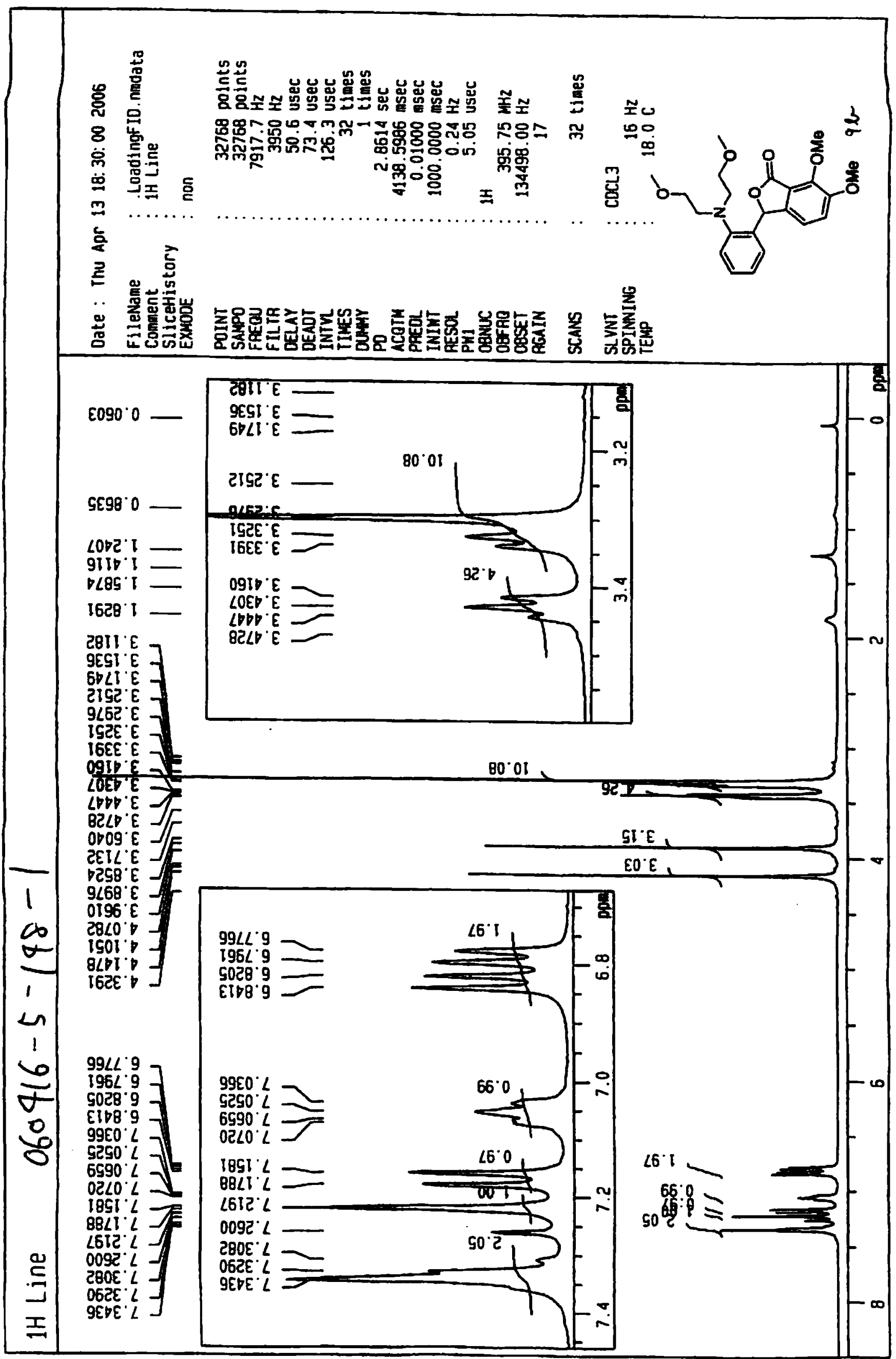




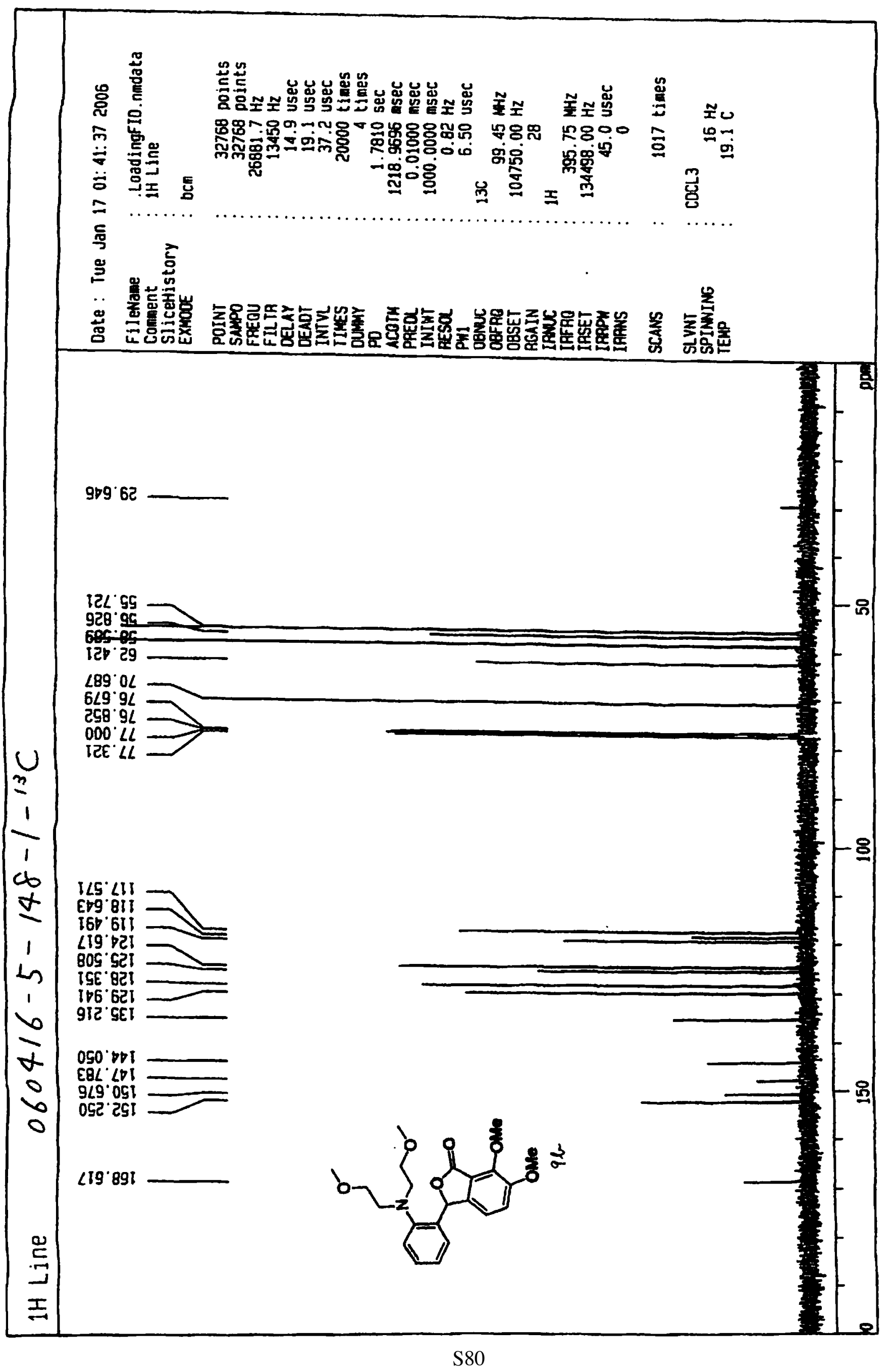




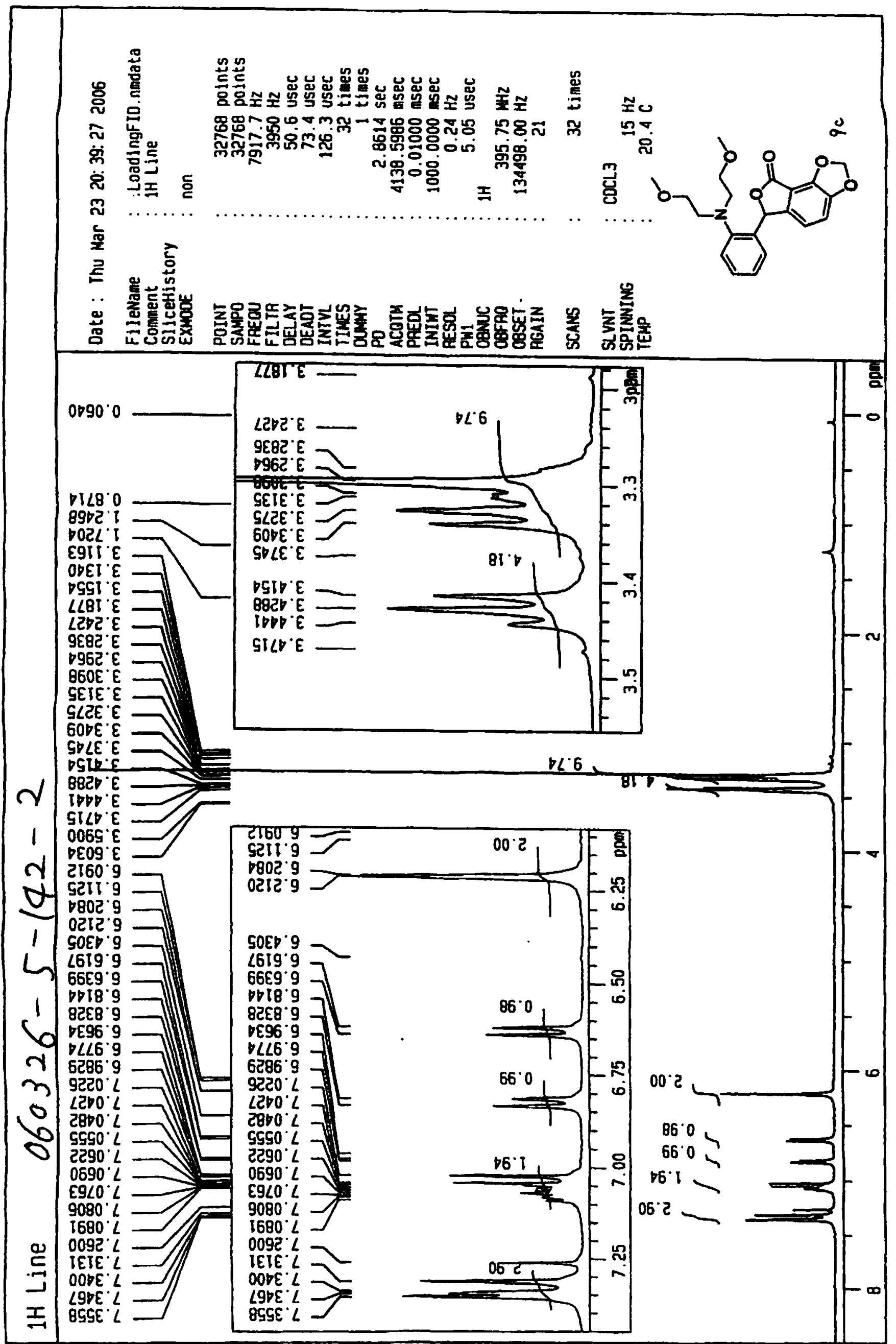




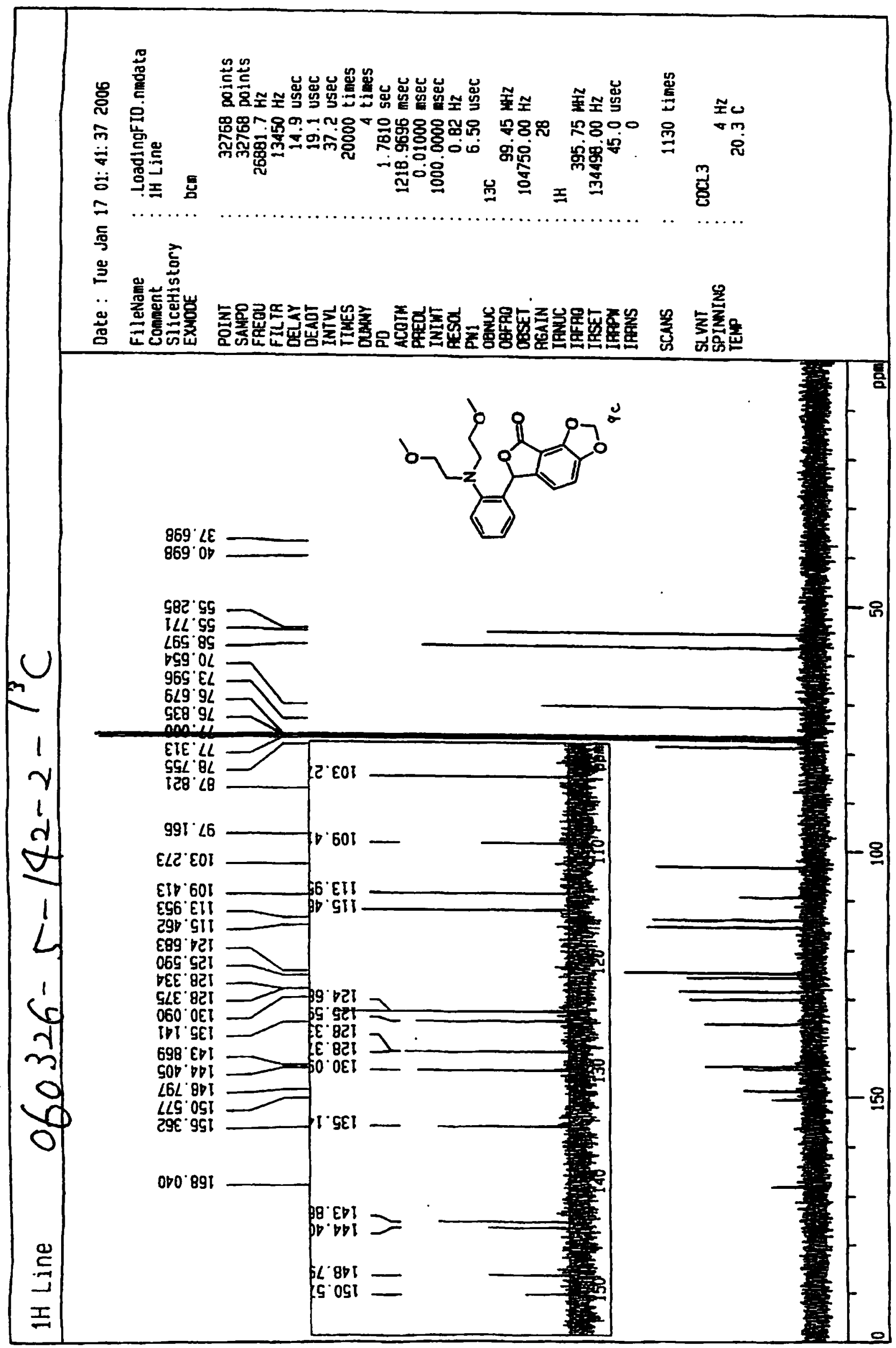

